\title{
Municipal and Commercial Equipment for Radiological Response and Recovery in an Urban Environment: State of Science, Research Needs, and Evaluation of Implementation towards Critical Infrastructure Resilience
}

Nuclear Engineering Division 


\begin{abstract}
About Argonne National Laboratory
Argonne is a U.S. Department of Energy laboratory managed by UChicago Argonne, LLC under contract DE-AC02-06CH11357. The Laboratory's main facility is outside Chicago, at 9700 South Cass Avenue, Argonne, Illinois 60439. For information about Argonne and its pioneering science and technology programs, see www.anl.gov.
\end{abstract}

\title{
DOCUMENT AVAILABILITY
}

Online Access: U.S. Department of Energy (DOE) reports produced after 1991 and a growing number of pre-1991 documents are available free via DOE's SciTech Connect (http://www.osti.gov/scitech/).

Reports not in digital format may be purchased by the public from the National Technical Information Service (NTIS):

U.S. Department of Commerce

National Technical Information Service

5301 Shawnee Road

Alexandria, VA 22312

unw.ntis.gov

Phone: (800) 553-NTIS (6847) or (703) 605-6000

Fax: (703) 605-6900

Email: orders@ntis.gov

Reports not in digital format are available to DOE and DOE contractors from:

U.S. Department of Energy

Office of Scientific and Technical Information

P.O. Box 62

Oak Ridge, TN 37831-0062

\section{Disclaimer}

This report was prepared as an account of work sponsored by an agency of the United States Government. Neither the United States Government nor any agency thereof, nor UChicago Argonne, LLC, nor any of their employees or officers, makes any warranty, express or implied, or assumes any legal liability or responsibility for the accuracy, completeness, or usefulness of any information, apparatus, product, or process disclosed, or represents that its use would not infringe privately owned rights. Reference herein to any specific commercial product, process, or service by trade name, trademark, manufacturer, or otherwise, does not necessarily constitute or imply its endorsement, recommendation, or favoring by the United States Government or any agency thereof. The views and opinions of document authors expressed herein do not necessarily state or reflect those of the United States Government or any agency thereof, Argonne National Laboratory, or UChicago Argonne, LLC.

\section{Disclaimer}

The U.S. Environmental Protection Agency (EPA) through its Office of Research and Development (ORD), in collaboration with the U.S. Department of Homeland Security's (DHS's) Science and Technology Directorate, funded and managed the research described. Note that this does not signify that the contents necessarily reflect the views of the Agency. Mention of trade names, products, or services does not convey official DHS or EPA approval, endorsement, or recommendation. 


\section{Municipal and Commercial Equipment for Radiological Response and Recovery in an Urban Environment: State of Science, Research Needs, and Evaluation of Implementation towards Critical Infrastructure Resilience}

by

Michael D. Kaminski

Nuclear Engineering Division

Argonne National Laboratory

Katrina M. McConkey

Booz Allen Hamilton

Matthew Magnuson and Sang Don Lee

U.S. Environmental Protection Agency

Office of Research and Development

National Homeland Security Research Center

Benjamin Stevenson and Orly Amir

National Urban Security Technology Laboratory

Department of Homeland Security

May 2018 



\section{CONTENTS}

ACRONYMS AND ABBREVIATIONS ..................................................................... vi

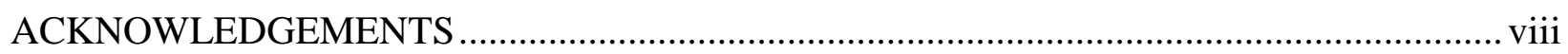

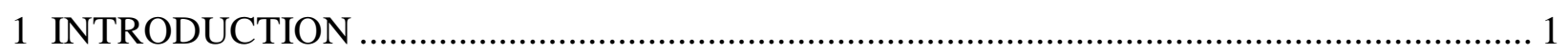

2 GENERAL KNOWLEDGE GAPS AND EXISTING GUIDANCE DOCUMENTS .............. 4

3 SUPPORT GOALS AND SCENARIOS EQUIPMENT/ TECHNOLOGY INFORMATION .......................................................................... 7

3.1 Support Goal: Survey and Monitoring …............................................................... 7

3.2 Support Goal: Mitigation of Received Dose to First Responders ................................ 16

3.3 Support Goal: Decontamination (Gross and Final) ................................................. 27

3.4 Support Goal: Waste Management ....................................................................... 35

3.5 Support Goal: Containment of Wastewater and Other Wastes ................................... 40

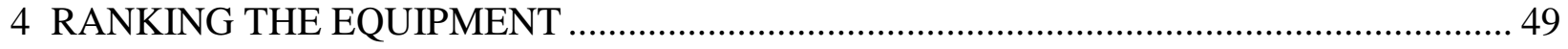

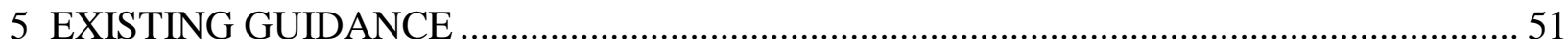

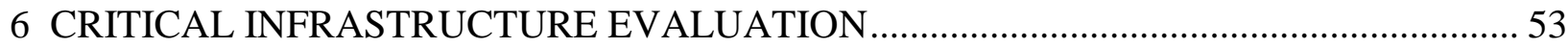

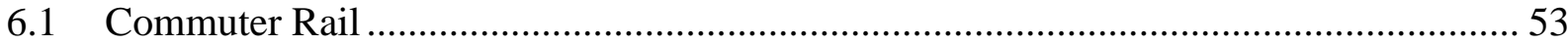

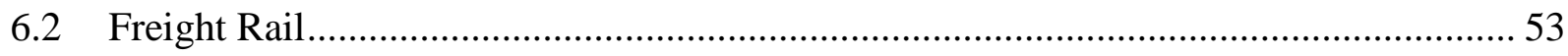

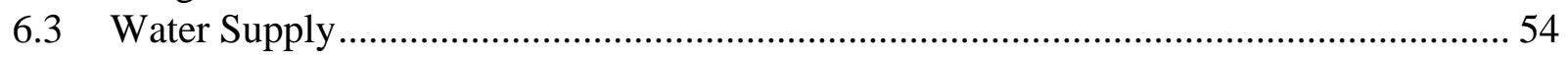

6.4 Waste Water Treatment Plants .............................................................................. 55

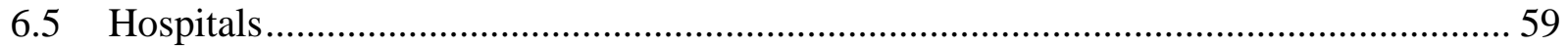

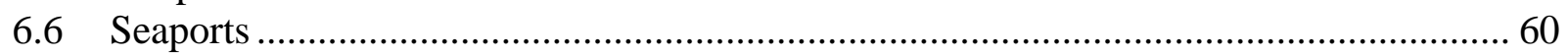

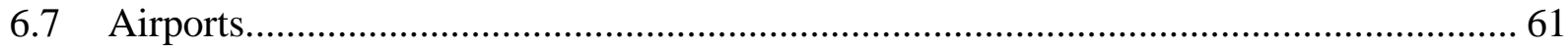

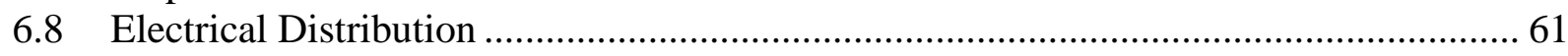

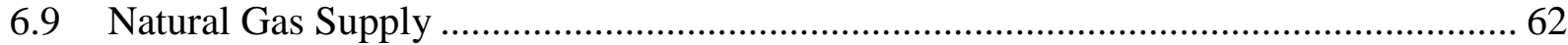

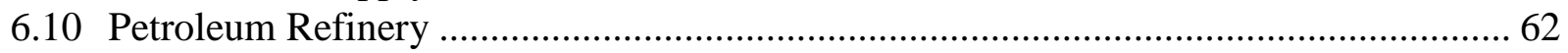

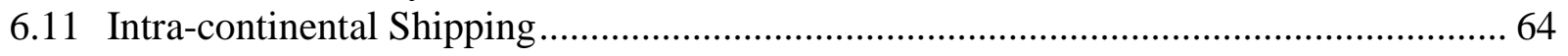

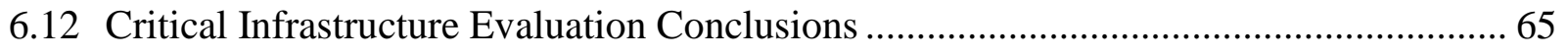

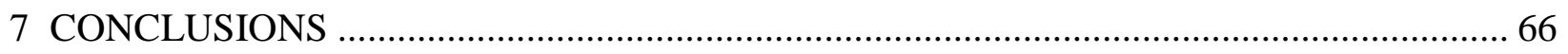

\section{FIGURES}

6-1 MWRD Stickney Plant flow diagram. ..................................................................... 56

6-2 A look from above the coarse screening operations............................................... 57

6-3 Water level view of the aerated grit tanks that can be used to remove the bulk of sequestering agents introduced in the sewer system or in the coarse screen houses....... 57

6-4 Close-up view of the collection trench in a drained aeration grit tank ........................ 58 


\section{FIGURES (CONT.)}

6-5 OCWD inflatable rubber dam spanning the Santa Ana River in Anaheim to divert river water that would otherwise flow to the ocean........................................ 59

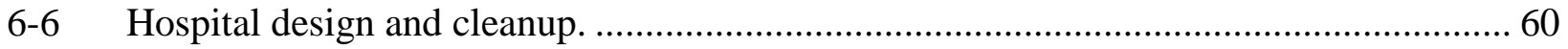

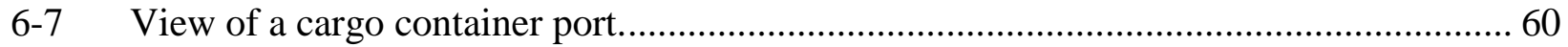

6-8 View of a large international airport located in the United States.............................. 61

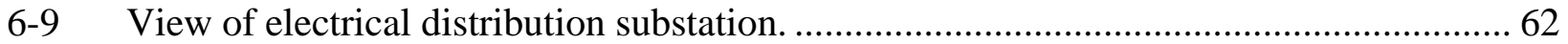

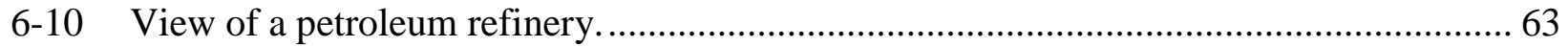

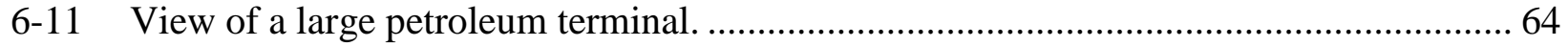

6-12 Views of inland waterway port and rail yard for the movement of intra-continental goods.

\section{TABLES}

2-1 Common Limitations in Our Ability to Use the Equipment/Technology for Its Proposed Purpose or Lack of Knowledge about Its Efficacy..................................... 4

3-1-1 Support Goal: Survey and Monitoring for Scenario 1 .............................................. 8

3-1-2 Support Goal: Survey and Monitoring for Scenario 2 ............................................ 9

3-1-3 Support Goal: Survey and Monitoring for Scenario 3 ............................................. 10

3-1-4 Support Goal: Survey and Monitoring for Scenario 4 ........................................... 11

3-1-5 Support Goal: Survey and Monitoring for Scenario 5 ............................................ 12

3-1-6 Support Goal: Survey and Monitoring for Scenario 6 ............................................. 13

3-1-7 Support Goal: Survey and Monitoring for Scenario 7 ............................................. 14

3-1-8 Support Goal Training: Survey and Monitoring for Scenarios 1-7 ............................. 15

3-2-1 Support Goal: Mitigation of Received Dose to First Responders for Scenario 1 ............ 17

3-2-2 Support Goal: Mitigation of Received Dose to First Responders for Scenario 2 ............ 18

3-2-3 Support Goal: Mitigation of Received Dose to First Responders for Scenario 3 ............ 19

3-2-4 Support Goal: Mitigation of Received Dose to First Responders for Scenario 4 ............ 21

3-2-5 Support Goal: Mitigation of Received Dose to First Responders for Scenario 5 ............ 22

3-2-6 Support Goal: Mitigation of Received Dose to First Responders for Scenario 6 ............ 23

3-2-7 Support Goal: Mitigation of Received Dose to First Responders for Scenario 7 ............ 24

3-2-8 Support Goal: Mitigation of Received Dose to First Responders for Scenario 8 ............ 25

3-2-9 Support Goal: Mitigation of Received Dose to First Responders for Scenario 9 ............ 25 


\section{TABLES (CONT.)}

3-2-10 Support Goal Training: Mitigation of Received Dose to First Responders for

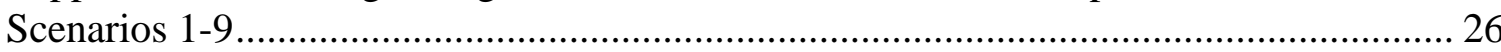

3-3-1 Support Goal: Decontamination (gross and final) for Scenario 1 1............................... 28

3-3-2 Support Goal: Decontamination (gross and final) for Scenario 2 2.............................. 30

3-3-3 Support Goal: Decontamination (gross and final) for Scenario 3 ............................... 31

3-3-4 Support Goal: Decontamination (gross and final) for Scenario 4 ................................ 32

3-3-5 Support Goal: Decontamination (gross and final) for Scenario 5 ............................... 33

3-3-6 Support Goal: Decontamination (gross and final) for Scenario 6................................. 34

3-3-7 Support Goal Training: Decontamination (gross and final) for Scenarios 1-6 ................ 34

3-4-1 Support Goal: Waste Management for Scenario 1 .................................................. 36

3-4-2 Support Goal: Waste Management for Scenario 2 ................................................... 37

3-4-3 Support Goal: Waste Management for Scenario 3 ..................................................... 38

3-4-4 Support Goal: Waste Management for Scenario 4 ....................................................... 39

3-4-5 Support Goal Training: Scenarios 1-4 _..................................................................... 39

3-5-1 Support Goal: Containment of Wastewater and Other Wastes for Scenario 1 ............... 41

3-5-2 Support Goal: Containment of Wastewater and Other Wastes for Scenario 2 ................ 42

3-5-3 Support Goal: Containment of Wastewater and Other Wastes for Scenario 3 ............... 43

3-5-4 Support Goal: Containment of Wastewater and Other Wastes for Scenario 4 ................ 44

3-5-5 Support Goal: Containment of Wastewater and Other Wastes for Scenario 5 ................ 45

3-5-6 Support Goal: Containment of Wastewater and Other Wastes for Scenario 6 ................ 46

3-5-7 Support Goal: Containment of Wastewater and Other Wastes for Scenarios 7 and 8..... 47

3-5-8 Support Goal Training: Containment of Wastewater and Other Wastes for

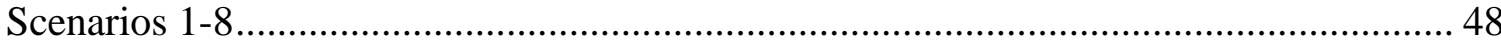

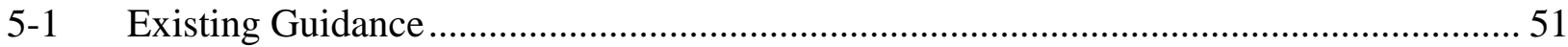




\section{ACRONYMS AND ABBREVIATIONS}

$\begin{array}{ll}\text { ALARA } & \text { As Low As Reasonably Achievable } \\ \text { BE } & \begin{array}{l}\text { Bench-scale Experiments. Includes bench- and laboratory-scale experiments } \\ \text { and computer modeling. }\end{array} \\ \text { COTS } & \text { Commercial Off the Shelf } \\ \text { DHS } & \begin{array}{l}\text { U.S. Department of Homeland Security } \\ \text { DOD }\end{array} \\ \text { DIY } & \text { U.S. Department of Defense } \\ \text { EPA } & \text { Environmental Protection Agency } \\ \text { EPD } & \text { Electronic Personal Dosimeters } \\ \text { FD } & \text { Fire Department } \\ \text { FRMAC } & \text { Federal Radiological Monitoring and Assessment Center } \\ \text { GCS } & \text { Grand Central Station } \\ \text { GIS } & \text { Geographic Information System } \\ \text { GPS } & \text { Global Positioning System } \\ \text { HP } & \text { Health Physicist } \\ \text { HEPA } & \text { High-Efficiency Particulate Air } \\ \text { HVAC } & \text { Heating, ventilation, and air conditioning } \\ \text { IBC } & \text { Intermediate Bulk Container } \\ \text { LR } & \text { Literature Review. Includes literature study, information gathering from } \\ \text { subject-matter experts, or other paper studies. } & \text { Metropolitan Transportation Authority } \\ \text { MTA } & \text { Metropolitan Water Reclamation District } \\ \text { MWRD } & \text { Sodium Iodide } \\ \text { NaI } & \text { National Vocational Qualification } \\ \text { NVQ } & \text { New York } \\ \text { NY } & \text { Orange County Water District } \\ \text { OCWD } & \text { Off Specification } \\ \text { Off spec } & \end{array}$




$\begin{array}{ll}\text { PPE } & \text { Personal Protective Equipment } \\ \text { PD } & \begin{array}{l}\text { Police Department } \\ \text { PE }\end{array} \\ \text { PTT } & \begin{array}{l}\text { Pilot-scale Experiments. Includes pilot- or large-scale experiments and } \\ \text { Push-To-Talk }\end{array} \\ \text { QA/QC } & \text { Quality Assurance/Quality Control } \\ \text { R5 } & \text { EPA Region 5 } \\ \text { RAP } & \text { Radiological Assistance Programs } \\ \text { ROSS } & \text { Radiological Operations Support Specialist } \\ \text { R\&D } & \text { Research and Development } \\ \text { RO } & \text { Reverse Osmosis } \\ \text { SME } & \text { Subject-Matter Expert } \\ \text { SQEP } & \text { Suitably Qualified and Experienced Person } \\ \text { TLD } & \text { Thermoluminescent Dose Meters } \\ \text { UK } & \text { United Kingdom } \\ \text { USB } & \text { Universal Serial Bus } \\ \text { WERF } & \text { Water Environment Research Foundation } \\ \text { WMD } & \text { Weapons of Mass Destruction }\end{array}$




\section{ACKNOWLEDGEMENTS}

The authors thank K. Hall (USEPA) and J. Harmon (Argonne) for technical editing that improved the organization and readability of this document. This work was supported by the Department of Homeland Security Science and Technology Directorate. The U.S. Environmental Protection Agency, through its Office of Research and Development, funded and collaborated in the research described here under Interagency Agreement 92380201 with Argonne National Laboratory. This document was reviewed in accordance with EPA policy prior to publication. Note that approval for publication does not signify that the contents necessarily reflect the views of the Agency. Mention of trade names or commercial products does not constitute endorsement or recommendation for use of a specific product. 


\section{INTRODUCTION}

The National Urban Security Technology Laboratory of the U.S. Department of Homeland Security (DHS), in collaboration with the U.S. Environmental Protection Agency's (EPA) National Homeland Security Research Center and Argonne National Laboratory, seeks to identify equipment that support methods to mitigate the effects of a radiological or nuclear release in the urban environment. This includes understanding the types of equipment that might be available and evaluating the efficacy of such equipment toward a number of potential response and recovery goals including monitoring, containing, and decontaminating contaminated structures, vehicles, and materials.

During the immediate, emergency phase of a response, it will be impossible to initiate activities other than establishing life-saving operations and securing a site for the safety of the public and first responders. This project is concerned with the period between securing of the site and the beginning of a federally organized cleanup effort. This period might be significant, as we have learned from the Japanese and their response time for the disaster at Fukushima. Processes and procedures for a timely response to a wide-area contamination event are not currently established around the country. Response planning of this type is still in the very early stages, and this project is part of this work.

This project focused on connecting with relevant emergency management and homeland security offices at the city, state, and regional level, including major cities across the United States, and with federal agencies. Within these offices, we consulted with subject matter experts (SMEs) and also evaluated commercial-off-the-shelf products. The result was a compilation and assessment of approaches to address potential contamination scenarios and the types of equipment assets that would or could assist in the response and recovery effort. We also documented what recommendations the SMEs suggested on how current equipment reserves can be best used or modified to improve their utility in radiological or nuclear mitigation.

This report contains a comprehensive list of proposed equipment to accomplish various missions or scenarios that might arise after a large-scale radiological contamination incident in an urban environment. Preliminary information was published in separate reports, Subject Matter Expert Workshop for the Use of Municipal and Commercial Equipment for Radiological Response and Recovery Summary Report: Argonne National Laboratory (ANL/NE-17/35) and Subject Matter Expert Workshop for the Use of Municipal and Commercial Equipment for Radiological Response and Recovery Summary Report: National Urban Security Technology Laboratory (ANL/NE-17/36). We attempted to include all relevant SMEs, stakeholders, and information resources. However, we recognize that gaps remain in our knowledge because regions vary in capabilities and assets, and input was missing from specific experts who were unable to attend and could not be reached for comment. We list these information gaps in Table 2-1. Moreover, we recognize that existing guidance and technical documents are already available for certain situations. Some examples are provided in Section 5, Existing Guidance, although this listing is not meant to be exhaustive. 
Potential response and recovery efforts were divided into five support goals (see tables in Section 3). The five support goals were as follows:

- Survey and monitoring of the contaminated area;

- Mitigation of received dose to first responders;

- Decontamination (gross and final) of buildings, vehicles, roadways, parks, and other surfaces;

- Waste management of solid waste generated during recovery operations; and

- Containment of wastewater and other waste generated during the response and recovery phases.

Within each support goal, we define several missions or scenarios that describe specific situations that require a response activity. Within each support goal sheet, next to each scenario, we summarize the general techniques and equipment suggested by the SMEs and our information gathering activities. For each piece of equipment, we briefly describe its proposed function, its advantages, and the potential limitations in its use, our knowledge of its function, or its efficacy to accomplish the task proposed in the scenario. Finally, we summarize the general research and development $(\mathrm{R} \& \mathrm{D})$ needs for items. These are further categorized as literature review (LR), bench-scale experiment (BE), and pilot-scale experiments (PE):

- LR - literature review. Includes literature study, information gathering from subjectmatter experts, or other paper studies.

- $\quad \mathrm{BE}$ - bench-scale experiment. Includes bench- and laboratory-scale experiments and computer modeling.

- $\quad \mathrm{PE}$ - pilot-scale experiment. Includes pilot- or large-scale experiments and demonstrations.

The information from Tables 3-1-1 to 3-5-8 was disseminated to SMEs so they could rank equipment and/or technologies that might have the most impact and could benefit from future work to better understand its efficacy. This future work may include the development of bestpractice guides, as well as laboratory and/or field-testing of municipal equipment. From their responses, we identified those equipment and technologies ranked highest (Section 4).

Another aspect of this report is to address the five support goals considering the potential needs of critical infrastructure. Critical infrastructures (e.g., government, health care, school, transportation, energy, communication, etc.) in the contaminated area must be restored quickly to minimize both direct and indirect impacts. For example, wide area contamination may pose a direct impact to the local community due to health impacts and denial of services, including possible relocation. Surrounding communities may also be affected indirectly by inhibiting people who travel to the community for work or personal activities or rely on services from the 
directly impacted area. Rapid decontamination methods will be needed for critical infrastructure to enable their continuous operation. This report provides a brief description of various critical infrastructure and information gathered on mitigating the effects of contamination. 


\section{GENERAL KNOWLEDGE GAPS AND EXISTING GUIDANCE DOCUMENTS}

This project identified common gaps across most or all of the support goals for use of equipment to accomplish various missions or scenarios that might arise after a large-scale radiological contamination incident in an urban environment (Table 2-1). We identified these gaps after reviewing the information gathered from various activities. These activities included two workshops attended by various local, state, regional, and federal agencies and by SMEs in the areas of response/recovery and management of equipment and personnel assets; telephone conversations; personal meetings; and feedback with SMEs. We attempted to include all the relevant SMEs and stakeholders and review pertinent documents. However, we recognize that gaps remain in our knowledge because of local, state, and regional variance in capabilities and assets, missing input from specific experts who were unable to attend or could not be reached for comment, and uncertainties in the performance of equipment for off-spec activities. Gaps specific to the use of a particular piece of equipment or technology and opportunities for R\&D are provided in Tables 3-1-1 through 3-5-8.

TABLE 2-1 Common Limitations in Our Ability to Use the Equipment/Technology for Its Proposed Purpose or Lack of Knowledge about Its Efficacy.

\begin{tabular}{|c|c|}
\hline Topic & Description of General Overarching Needs \\
\hline $\begin{array}{l}\text { Critical } \\
\text { Infrastructure }\end{array}$ & $\begin{array}{l}\text { - The predicted effects of contamination on critical infrastructure need to be } \\
\text { assessed (e.g., fate and transport of contaminant, exposure potential for } \\
\text { public and workers). Methods of decontamination need to be assessed. } \\
\text { Guidance on use of measurement instruments specific to critical } \\
\text { infrastructure and geographic information system (GIS) integration of some } \\
\text { of the equipment listed will be necessary. } \\
\text { - Key input is missing regarding capabilities and assets available to critical } \\
\text { infrastructure that includes local, state, and regional variations. This includes } \\
\text { but is not limited to infrastructure associated with drinking water supply and } \\
\text { distribution networks, wastewater treatment, sewers, tunnels and bridges, } \\
\text { transportation/highway authority, airport authority, ports, fleet and facility } \\
\text { management, hospitals, energy (electricity, natural gas, fuel), and } \\
\text { communication centers. }\end{array}$ \\
\hline Data Sharing & $\begin{array}{l}\text { During a response and recovery effort, data may be gathered from response } \\
\text { teams and then handed over to recovery teams. The data might be further } \\
\text { distributed to state and local agencies. Each agency may have different } \\
\text { platforms for data management, and the visualizations they generate to } \\
\text { communicate with the public might be confusing. The data (i.e., data } \\
\text { management platforms and GIS integration) need to be compatible between } \\
\text { agencies and reconcile issues related to quality assurance/quality control } \\
\text { (QA/QC). }\end{array}$ \\
\hline
\end{tabular}


TABLE 2-1 (Cont.)

\begin{tabular}{|c|c|}
\hline Topic & Description of General Overarching Needs \\
\hline GIS Integration & $\begin{array}{l}\text { - Although many agencies use GIS platforms, all systems may not be } \\
\text { compatible with each other. Therefore, data gathered during the response } \\
\text { and recovery effort needs to be made compatible with existing GIS data } \\
\text { management systems. } \\
\text { - The U.S. Department of Defense (DOD) used GIS integration during the } \\
\text { responses to Hurricane Sandy and other natural disasters through their joint } \\
\text { task force-civil support teams. Discussions of DOD capabilities are needed } \\
\text { to better understand the challenges associated with integrating various GIS } \\
\text { platforms during a recovery effort. }\end{array}$ \\
\hline $\begin{array}{l}\text { Data to Support } \\
\text { Off-Spec Use }\end{array}$ & 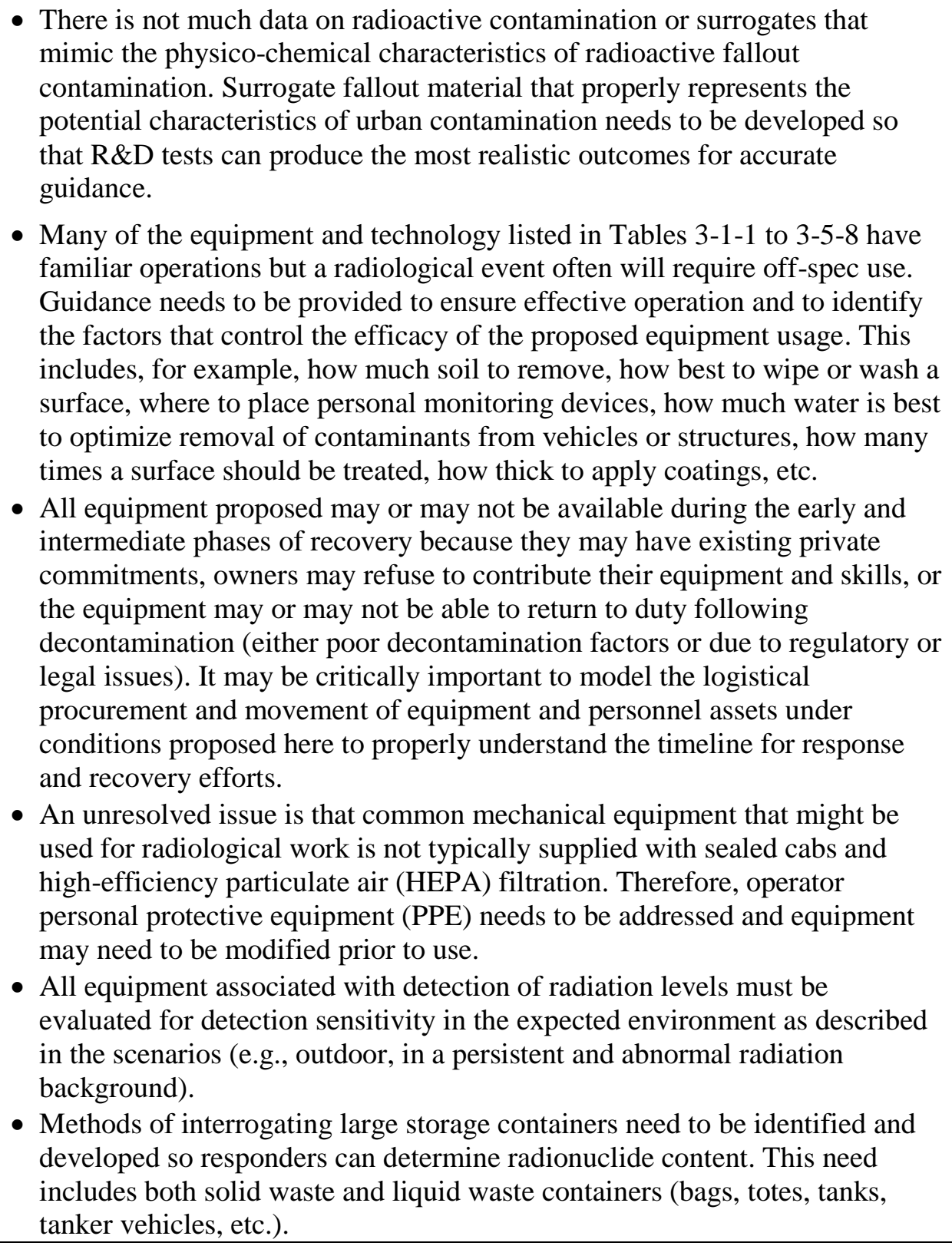 \\
\hline
\end{tabular}


TABLE 2-1 (Cont.)

\begin{tabular}{|l|l|}
\hline \multicolumn{1}{|c|}{ Topic } & \multicolumn{1}{c|}{ Description of General Overarching Needs } \\
\hline $\begin{array}{l}\text { Data to Support } \\
\text { Off-Spec Use } \\
\text { (Cont.) }\end{array}$ & $\begin{array}{l}\text { - For do-it-yourself (DIY) equipment, }{ }^{1} \text { best practices guidance is not } \\
\text { available. Such guidance would need to be published to reduce risk and } \\
\text { improve efficacy. }\end{array}$ \\
\hline - Rules and regulations related to transporting potentially-contaminated \\
materials in vehicles not licensed for such use need to be reviewed. \\
- PPE requirements need to be established for handling potentially radioactive \\
materials. \\
Training
\end{tabular}

${ }^{1}$ DIY may include equipment and techniques that may be used by individual homeowners or volunteer cleanup teams. 


\section{SUPPORT GOALS AND SCENARIOS EQUIPMENT/TECHNOLOGY INFORMATION}

This section describes the five support goals and the scenarios posed within them. Tables 3-1-1 through 3-5-8 summarize the equipment and technologies proposed for various activities that might arise after a large-scale radiological contamination incident in an urban environment. Also, briefly described are the perceived advantages and known limitations of each and the suggested research and development needed to support the proposed use of the equipment or technology. These suggested needs are broadly categorized as literature reviews (LR) of existing documents, studies, or consultation with subject matter experts (SME), benchtop experiments (BE), and pilot-scale experiments (PE). It is expected that following these types of studies, sufficient data to develop specific guidance on the use of the equipment or technology and estimated efficacy for the proposed activities would be collected. Further, general observations were raised by the SMEs during the gathering of this information, and these ideas are captured either in the summary section of each table or in the last two rows of each table.

\subsection{SUPPORT GOAL: SURVEY AND MONITORING}

Contamination levels in affected areas need to be monitored, perhaps for an extended time, to understand the radiation dose to workers and residents and the evolving levels of contamination level over time. What types of municipal and commercial equipment can enhance surveys and monitoring of contamination? (Note that traditional survey monitoring equipment such as film badges, portable survey monitors, and gamma-ray spectrometers already in place with response vehicles or personnel will not be assessed because these are specialized pieces of equipment that are already accounted in the first responders' procedures.) Examples include measuring contamination in air filters from garbage trucks, delivery trucks, police cars, and firetrucks that have well-defined routes. These vehicles can be tracked using global positioning system (GPS) monitoring to understand the spatial distribution of airborne contamination, or traditional survey equipment can be attached to other vehicles that have well-defined routes. Tables 3-1-1 to 3-1-8 present scenarios related to survey and monitoring, possible responses to the scenarios, and possible equipment/technology used in responding, including its advantages, limitations, and $\mathrm{R} \& \mathrm{D}$ needs. 
TABLE 3-1-1 Support Goal: Survey and Monitoring for Scenario 1

\begin{tabular}{|c|c|c|c|c|c|c|c|c|c|c|}
\hline \multirow{2}{*}{$\begin{array}{c}\text { Scenario } \\
\text { Description } \\
\end{array}$} & \multirow{2}{*}{ Summary } & \multirow{2}{*}{ Category } & \multicolumn{8}{|c|}{ Column Number } \\
\hline & & & 1 & 2 & 3 & 4 & 5 & 6 & 7 & 8 \\
\hline \multirow{5}{*}{$\begin{array}{l}\text { Scenario 1: } \\
\text { Measure, on a } \\
\text { regular basis, } \\
\text { the } \\
\text { contamination } \\
\text { levels in areas } \\
\text { initially affected } \\
\text { by radioactive } \\
\text { contamination } \\
\text { (fallout deposits } \\
\text { of radioactive } \\
\text { material) in } \\
\text { terms of } \\
\text { external dose at } \\
\text { the street level } \\
\text { (the radiation } \\
\text { levels at the } \\
\text { street from } \\
\text { external gamma } \\
\text { and beta } \\
\text { radiation). What } \\
\text { equipment can } \\
\text { provide such } \\
\text { measurements? } \\
\text { What tools are } \\
\text { available to } \\
\text { develop maps of } \\
\text { contamination } \\
\text { with this data? }\end{array}$} & \multirow{5}{*}{ 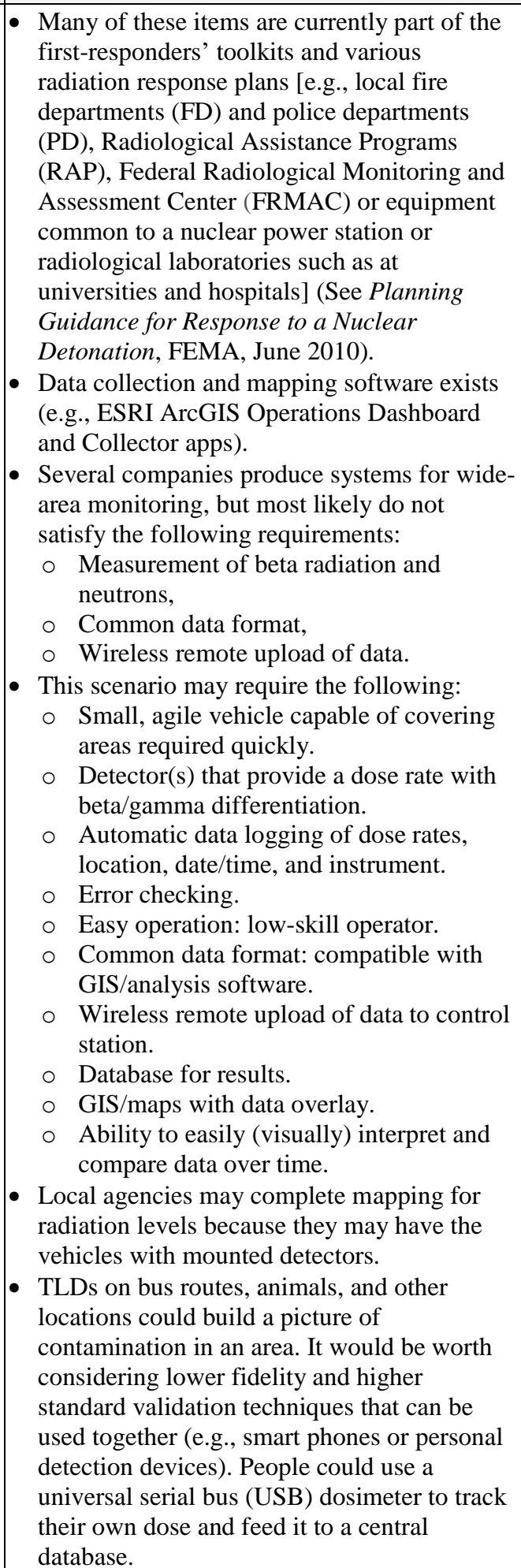 } & EQUIPMENT & \begin{tabular}{|l} 
Smart phone \\
radiation detectors.
\end{tabular} & $\begin{array}{l}\text { Thermoluminescent } \\
\text { dose meters } \\
\text { (TLDs). }\end{array}$ & $\begin{array}{l}\text { Electronic } \\
\text { personal } \\
\text { dosimeters (EPDs). }\end{array}$ & Electronic data logging. & $\begin{array}{l}\text { Two-dimensional } \\
\text { (2D) mapping } \\
\text { gamma camera. }\end{array}$ & $\begin{array}{l}\text { Vehicle-mounted large- } \\
\text { aperture detectors (e.g., } \\
\text { NaI). }\end{array}$ & $\begin{array}{l}\text { Manned, detailed } \\
\text { measurement surveys. }\end{array}$ & Small, agile vehicle. \\
\hline & & DESCRIPTION & $\begin{array}{l}\text { Detects radiation } \\
\text { with a metal-oxide } \\
\text { semiconductor chip. }\end{array}$ & $\begin{array}{l}\text { Measures beta and } \\
\text { gamma doses at } \\
\text { fixed or mobile } \\
\text { locations. }\end{array}$ & $\begin{array}{l}\text { Measures gamma } \\
\text { doses at a range of } \\
\text { fixed or mobile } \\
\text { locations. }\end{array}$ & $\begin{array}{l}\text { Provides continuous } \\
\text { gamma and neutron } \\
\text { monitoring using sodium } \\
\text { iodide (NaI) detector. }\end{array}$ & $\begin{array}{l}\text { Identifies hotspots } \\
\text { in a general } \\
\text { radiation field. } \\
\text { May be able to } \\
\text { produce a 3-D } \\
\text { rendering of work } \\
\text { area. }\end{array}$ & $\begin{array}{l}\text { Mobile detectors for } \\
\text { mapping contamination. }\end{array}$ & $\begin{array}{l}\text { Portable instruments for } \\
\text { trained operators. }\end{array}$ & $\begin{array}{l}\text { Ground-based or aerial } \\
\text { vehicles outfitted with } \\
\text { detectors. }\end{array}$ \\
\hline & & ADVANTAGES & $\begin{array}{l}\text { Existing network of } \\
\text { phones, mobile. }\end{array}$ & $\begin{array}{l}\text { Large numbers } \\
\text { commercial-off-the- } \\
\text { shelf (COTS), low } \\
\text { cost, fixed or } \\
\text { mobile usage. }\end{array}$ & $\begin{array}{l}\text { Large numbers } \\
\text { COTS, low cost, } \\
\text { fixed or mobile } \\
\text { usage, large } \\
\text { sensitivity range } \\
(0.1 \mu \mathrm{Sv} / \mathrm{h} \text { to } \\
10 \mathrm{~Sv} / \mathrm{h}) .\end{array}$ & $\begin{array}{l}\text { COTS, large sensitivity } \\
\text { range }(0.1 \mu \mathrm{S} v / \mathrm{h} \text { to } \\
10 \mathrm{~S} v / \mathrm{h}) \text {, fixed or mobile } \\
\text { usage. }\end{array}$ & $\begin{array}{l}\text { Fixed or mobile, } \\
\text { hotspot } \\
\text { identification (ID), } \\
\text { isotopic ID. }\end{array}$ & $\begin{array}{l}\text { GIS integrated, fixed or } \\
\text { mobile, isotopic ID. }\end{array}$ & $\begin{array}{l}\text { Large numbers COTS, } \\
\text { mobile. }\end{array}$ & Mobile, large coverage rate. \\
\hline & & LIMITATIONS & \begin{tabular}{|l} 
QA/QC, high \\
threshold \\
( 1 10 S S Vh/h), GIS \\
integration apps, \\
gamma only. \\
\end{tabular} & $\begin{array}{l}\text { High threshold, not } \\
\text { real-time, no GIS } \\
\text { integration, } \\
\text { beta/gamma but no } \\
\text { alpha, limited } \\
\text { readers available. }\end{array}$ & $\begin{array}{l}\text { No GIS integration, } \\
\text { gamma only. }\end{array}$ & $\begin{array}{l}\text { No GIS integration, not } \\
\text { rugged enough for } \\
\text { outdoors }\end{array}$ & $\begin{array}{l}\text { Limited quantity } \\
\text { available, high } \\
\text { threshold, gamma } \\
\text { only. Some models } \\
\text { may not have GIS } \\
\text { integration. }\end{array}$ & $\begin{array}{l}\text { Limited quantity available, } \\
\text { gamma only. }\end{array}$ & $\begin{array}{l}\text { No GIS integration, } \\
\text { significant manpower, } \\
\text { background radiation } \\
\text { interference, access to } \\
\text { private premises. }\end{array}$ & $\begin{array}{l}\text { Available numbers, GIS } \\
\text { integration, skilled } \\
\text { operators. }\end{array}$ \\
\hline & & R\&D NEEDS & $\begin{array}{l}\text { Guidance on use } \\
\text { (LR). Practicality for } \\
\text { immature technology } \\
\text { (LR, BE). GIS } \\
\text { integration tools } \\
\text { (LR, BE). }\end{array}$ & $\begin{array}{l}\text { Guidance on use } \\
\text { (LR). Integrate } \\
\text { lower fidelity and } \\
\text { higher standard } \\
\text { validation } \\
\text { techniques (e.g., } \\
\text { smart phones, TLD, } \\
\text { or EPD) (LR, BE, } \\
\text { PE). }\end{array}$ & $\begin{array}{l}\text { Guidance on use } \\
\text { (LR). GIS } \\
\text { integration tool } \\
\text { (LR, BE). }\end{array}$ & $\begin{array}{l}\text { Guidance on use (LR). } \\
\text { GIS integration tool } \\
\text { (LR, BE). }\end{array}$ & $\begin{array}{l}\text { Guidance on use } \\
\text { (LR). GIS } \\
\text { integration tool } \\
\text { (LR, BE). Survey } \\
\text { of inventory (LR). }\end{array}$ & Survey of inventory (LR). & $\begin{array}{l}\text { Guidance on use (LR). } \\
\text { GIS integration tool (LR, } \\
\text { BE) }\end{array}$ & $\begin{array}{l}\text { Survey of inventory (LR, } \\
\text { BE). Compatible radiation } \\
\text { detectors (BE, PE). }\end{array}$ \\
\hline
\end{tabular}

All images are personal photographs or courtesy of Shutterstock. 
TABLE 3-1-2 Support Goal: Survey and Monitoring for Scenario 2

\begin{tabular}{|c|c|c|c|c|c|c|c|c|}
\hline Scenario Description & Summary & Category & \multirow{2}{*}{\multicolumn{6}{|c|}{ Column Number }} \\
\hline & & & 1 & & 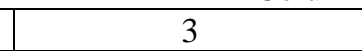 & 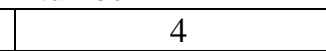 & 5 & 6 \\
\hline \multirow{5}{*}{$\begin{array}{l}\text { Scenario 2: } \\
\text { Measure, on a regular basis, } \\
\text { the contamination levels in } \\
\text { areas initially affected by } \\
\text { radioactive contamination } \\
\text { (fallout deposits of } \\
\text { radioactive material) in } \\
\text { terms of external dose } \\
\text { within residences and } \\
\text { businesses (the radiation } \\
\text { levels within homes, } \\
\text { apartments, or business } \\
\text { offices from external } \\
\text { gamma and beta radiations). } \\
\text { What equipment can be } \\
\text { used to provide such } \\
\text { measurement? What tools } \\
\text { are available to develop } \\
\text { maps of contamination with } \\
\text { this data? }\end{array}$} & \multirow{5}{*}{$\begin{array}{l}\text { - Most SMEs believe that monitoring within } \\
\text { homes and individual businesses will be more } \\
\text { difficult than external monitoring. } \\
\text { - Confidence in the dose data that is } \\
\text { disseminated to the public is necessary. For } \\
\text { data collected by expert organizations like } \\
\text { FRMAC, this is not an issue. However, if we } \\
\text { provide detection systems onto people, then } \\
\text { we must have confidence in their response. } \\
\text { - In some cases, these would represent low- } \\
\text { fidelity data. However, this data may } \\
\text { represent a very thorough cross-section of the } \\
\text { affected environment. Guidance on use and } \\
\text { GIS integration of the equipment listed will } \\
\text { be necessary. }\end{array}$} & EQUIPMENT & $\begin{array}{l}\text { Smart phone radiation } \\
\text { detectors. }\end{array}$ & $\begin{array}{l}\text { Environmental } \\
\text { TLDs or EPDs. }\end{array}$ & $\begin{array}{l}\text { Instadose by MIRION } \\
\text { Technologies. }\end{array}$ & $\begin{array}{l}\text { Manned, detailed } \\
\text { measurement surveys. }\end{array}$ & $\begin{array}{l}\text { Tacky mats for human } \\
\text { traffic. }\end{array}$ & $\begin{array}{l}\text { Two-dimensional (2D) } \\
\text { mapping gamma } \\
\text { camera. }\end{array}$ \\
\hline & & DESCRIPTION & $\begin{array}{l}\text { Detects radiation with : } \\
\text { metal-oxide } \\
\text { semiconductor chip. }\end{array}$ & $\begin{array}{l}\text { Measures beta and } \\
\text { gamma doses at a } \\
\text { range of fixed or } \\
\text { mobile locations. }\end{array}$ & $\begin{array}{l}\text { USB dosimeters to } \\
\text { deploy to many types of } \\
\text { vehicles, animals, and } \\
\text { structures. Can be given } \\
\text { to people to track their } \\
\text { own dose and send data } \\
\text { to a central database. }\end{array}$ & $\begin{array}{l}\text { Portable instruments } \\
\text { for trained operators. }\end{array}$ & $\begin{array}{l}\text { Controls loose } \\
\text { contamination at } \\
\text { entrances to buildings } \\
\text { or rooms. }\end{array}$ & $\begin{array}{l}\text { Identifies hotspots in a } \\
\text { general radiation field. } \\
\text { May be able to produce } \\
\text { a 3-D rendering of work } \\
\text { area. } \\
\text { The EPA (R5) is } \\
\text { working on indoor } \\
\text { mapping techniques. } \\
\text { Instruments use LIDAR } \\
\text { to view and map } \\
\text { interiors with attached } \\
\text { radiation equipment. }\end{array}$ \\
\hline & & ADVANTAGES & $\begin{array}{l}\text { Existing network of } \\
\text { phones. }\end{array}$ & $\begin{array}{l}\text { Large numbers } \\
\text { COTS, low cost, } \\
\text { fixed or mobile } \\
\text { usage. }\end{array}$ & $\begin{array}{l}\text { Cheap enough to } \\
\text { deploy. }\end{array}$ & $\begin{array}{l}\text { Large numbers } \\
\text { COTS. }\end{array}$ & $\begin{array}{l}\text { Different sizes and } \\
\text { colors, low cost, large } \\
\text { numbers COTS. }\end{array}$ & $\begin{array}{l}\text { Fixed or mobile, hotspo } \\
\text { ID, isotopic } \\
\text { identification. }\end{array}$ \\
\hline & & LIMITATIONS & $\begin{array}{l}\text { QA/QC, high threshold } \\
(\sim 10 \mu \text { Sv/h), GIS } \\
\text { integration apps, } \\
\text { gamma only. }\end{array}$ & $\begin{array}{l}\text { High threshold, not } \\
\text { real-time, no GIS } \\
\text { integration, } \\
\text { beta/gamma but no } \\
\text { alpha, limited } \\
\text { readers. }\end{array}$ & $\begin{array}{l}\text { No GIS integration, not } \\
\text { rugged for outdoors. }\end{array}$ & $\begin{array}{l}\text { No GIS integration, } \\
\text { manpower, } \\
\text { background radiation } \\
\text { interference, } \\
\text { ruggedness, access to } \\
\text { private premises. }\end{array}$ & $\begin{array}{l}\text { Handling and } \\
\text { disposal. }\end{array}$ & $\begin{array}{l}\text { Limited quantity } \\
\text { available, high } \\
\text { threshold, } \gamma \text { only. Some } \\
\text { models may not have } \\
\text { GIS integration. }\end{array}$ \\
\hline & & R\&D NEEDS & $\begin{array}{l}\text { Guidance on use (LR). } \\
\text { Practicality for } \\
\text { immature technology } \\
\text { (LR, BE). GIS } \\
\text { integration tools } \\
\text { (LR, BE). }\end{array}$ & $\begin{array}{l}\text { Guidance on use } \\
\text { (LR). Integrate } \\
\text { lower fidelity and } \\
\text { higher standard } \\
\text { validation } \\
\text { techniques (e.g., } \\
\text { smart phones, TLD, } \\
\text { or EPD) (LR, BE, } \\
\text { PE). }\end{array}$ & $\begin{array}{l}\text { Guidance on use (LR). } \\
\text { GIS integration tools } \\
\text { (LR, BE). }\end{array}$ & $\begin{array}{l}\text { Guidance on use (LR) } \\
\text { GIS integration tools } \\
\text { (LR, BE) }\end{array}$ & $\begin{array}{l}\text { Best practice } \\
\text { guidance (LR). } \\
\text { Removal efficiency } \\
\text { testing (BE). }\end{array}$ & $\begin{array}{l}\text { Guidance on use (LR). } \\
\text { GIS integration tool } \\
\text { (LR, BE). Survey of } \\
\text { inventory (LR). }\end{array}$ \\
\hline
\end{tabular}

All images are personal photographs or courtesy of Shutterstock. 
TABLE 3-1-3 Support Goal: Survey and Monitoring for Scenario 3

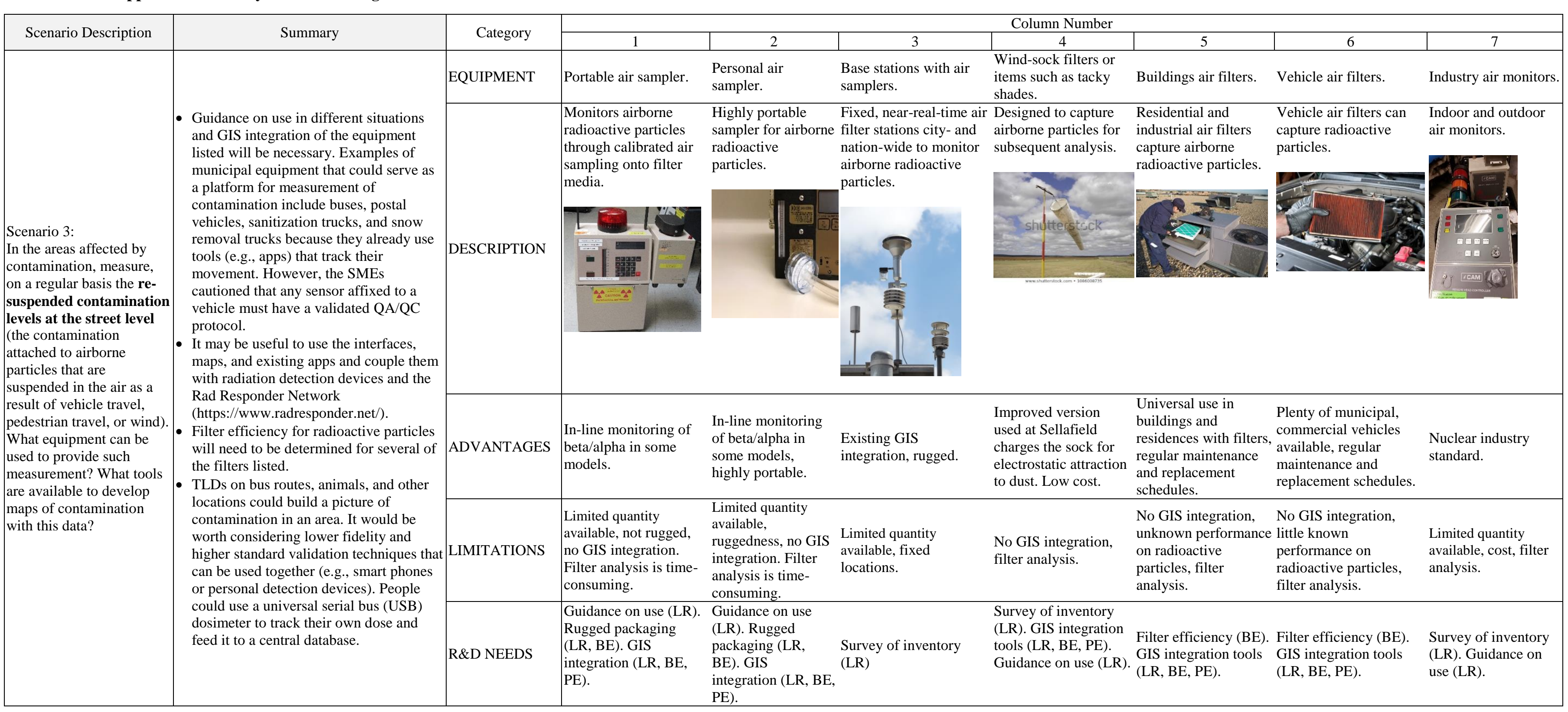


TABLE 3-1-4 Support Goal: Survey and Monitoring for Scenario 4

\begin{tabular}{|c|c|c|c|c|c|c|c|c|c|}
\hline Scenario & & & \multicolumn{7}{|c|}{ Column Number } \\
\hline Description & Summary & Category & 1 & 2 & 3 & 4 & 5 & 6 & 7 \\
\hline \multirow{5}{*}{$\begin{array}{l}\text { Scenario 4: } \\
\text { Track contamination } \\
\text { from the initial deposit } \\
\text { area to other areas via } \\
\text { vehicle and personnel } \\
\text { transport. What } \\
\text { equipment and methods } \\
\text { can be used to identify } \\
\text { these contaminated } \\
\text { egress routes? For } \\
\text { instance, can vehicles } \\
\text { originating from a } \\
\text { contaminated zone be } \\
\text { tracked using existing } \\
\text { highway cameras? }\end{array}$} & \multirow{5}{*}{$\begin{array}{l}\text { Much, if not all, of the discussion on general } \\
\text { - surveying from scenarios } 1-3 \text { applies here. } \\
\text { Monitoring and decontaminating, where } \\
\text { appropriate, of vehicles exiting the } \\
\text { contaminated areas would be required. To } \\
\text { monitor vehicles, filters from vehicle heating, } \\
\text { ventilation, and air conditioning (HVAC) } \\
\text { systems can be pulled. In addition, sanitation } \\
\text { trucks currently pass through portal monitors } \\
\text { before entering waste transfer stations. } \\
\text { - To monitor and control contamination on } \\
\text { private vehicles, portal monitors or portable } \\
\text { speed bump systems at strategic locations } \\
\text { (tunnel/bridge entrances, expressway ramps, } \\
\text { tollways) can be used. Contaminated cars can } \\
\text { be diverted through vehicle wash units (or ad } \\
\text { hoc systems) used for garbage trucks and } \\
\text { buses. } \\
\text { - Could also drive vehicles over tacky mats to } \\
\text { remove contamination from tires. Mats can be } \\
\text { monitored to show trends and areas most } \\
\text { affected. } \\
\text { - A massive distribution of handheld dosimetry } \\
\text { devices around cities, neighborhoods, and } \\
\text { subdivisions could be used. Real-time data } \\
\text { output could be directed to stakeholders (e.g., } \\
\text { homeowner association websites) to maintain } \\
\text { awareness and transparency (public trust). } \\
\text { - Concerns would include maintaining QA/QC } \\
\text { of data originating from untrained users and } \\
\text { integrating GIS into equipment used for } \\
\text { detection or tracking. }\end{array}$} & EQUIPMENT & $\begin{array}{l}\text { Tacky mats for } \\
\text { vehicles. }\end{array}$ & $\begin{array}{l}\text { Airborne wide-area } \\
\text { monitoring. }\end{array}$ & $\begin{array}{l}\text { Mobile phone with } \\
\text { location tracking }\end{array}$ & $\begin{array}{l}\text { Automatic plate } \\
\text { number recognition } \\
\text { (tollways). }\end{array}$ & $\begin{array}{l}\text { Portable monitors on } \\
\text { tracked vehicles. }\end{array}$ & Vehicle air filters. & $\begin{array}{l}\text { Radiation speed bump } \\
\text { detectors. }\end{array}$ \\
\hline & & DESCRIPTION & $\begin{array}{l}\text { Controls loose } \\
\text { contamination and can } \\
\text { be adapted to remove } \\
\text { rad particles from tires. }\end{array}$ & $\begin{array}{l}\text { Detector systems on } \\
\text { helicopter or } \\
\text { unmanned aerial } \\
\text { vehicle can map } \\
\text { contamination } \\
\text { quickly. }\end{array}$ & $\begin{array}{l}\text { Can track people in } \\
\text { contaminated area. }\end{array}$ & $\begin{array}{l}\text { Can track suspected } \\
\text { contaminated vehicles } \\
\text { for follow-up (long- } \\
\text { term) assessment. }\end{array}$ & $\begin{array}{l}\text { Monitors added to } \\
\text { select vehicles can } \\
\text { provide quality data } \\
\text { to assess } \\
\text { contamination on }\end{array}$ & $\begin{array}{l}\text { Vehicle air filters can } \\
\text { capture radioactive } \\
\text { particles. }\end{array}$ & $\begin{array}{l}\text { Real-time detection to } \\
\text { segregate } \\
\text { contaminated vehicles. }\end{array}$ \\
\hline & & ADVANTAGES & $\begin{array}{l}\text { Different sizes and } \\
\text { colors, low cost, large } \\
\text { numbers COTS. }\end{array}$ & $\begin{array}{l}\text { Proven asset for } \\
\text { immediate and } \\
\text { prolonged survey } \\
\text { (e.g., Airborne } \\
\text { Radiological } \\
\text { Enhanced-sensor } \\
\text { System program } \\
\text { under DHS). }\end{array}$ & $\begin{array}{l}\text { Existing network of } \\
\text { phones, mobile. }\end{array}$ & $\begin{array}{l}\text { Local use, proven } \\
\text { system. }\end{array}$ & $\begin{array}{l}\text { Select vehicles for } \\
\text { best coverage, better } \\
\text { QA/QC control. }\end{array}$ & $\begin{array}{l}\text { Plenty of municipal } \\
\text { commercial vehicles } \\
\text { available, undergoing } \\
\text { regular maintenance. }\end{array}$ & Mobile. \\
\hline & & LIMITATIONS & $\begin{array}{l}\text { Handling and disposal, } \\
\text { manpower for change- } \\
\text { out, small footprint } \\
\text { compared to vehicles. }\end{array}$ & $\begin{array}{l}\text { Low resolution, } \\
\text { gamma radiation } \\
\text { only. }\end{array}$ & $\begin{array}{l}\text { Data access, privacy } \\
\text { concerns. }\end{array}$ & $\begin{array}{l}\text { How to link plate } \\
\text { information to } \\
\text { suspected } \\
\text { contamination? } \\
\text { Radiation monitor is } \\
\text { not integrated. }\end{array}$ & $\begin{array}{l}\text { Vehicle selection, } \\
\text { trained operators, GIS } \\
\text { tracking, gamma and } \\
\text { possibly beta only. }\end{array}$ & $\begin{array}{l}\text { No GIS integration, } \\
\text { little known } \\
\text { performance on } \\
\text { radioactive particles, } \\
\text { filter analysis. }\end{array}$ & $\begin{array}{l}\text { Only one supplier, } \\
\text { gamma only. }\end{array}$ \\
\hline & & R\&D NEEDS & $\begin{array}{l}\text { Practicality for outdoor } \\
\text { vehicle use (LR). } \\
\text { Removal efficiency } \\
\text { (BE, PE). }\end{array}$ & $\begin{array}{l}\text { Understand latest } \\
\text { capabilities (LR). } \\
\text { Rad detection } \\
\text { integration (PE). }\end{array}$ & $\begin{array}{l}\text { Practicality given } \\
\text { privacy concerns (LR). }\end{array}$ & $\begin{array}{l}\text { Rad detection } \\
\text { integration and } \\
\text { practicality (LR, PE). }\end{array}$ & $\begin{array}{l}\text { Guidance to } \\
\text { determine best } \\
\text { vehicles (LR). GIS } \\
\text { integration tools (LR, } \\
\text { PE). }\end{array}$ & $\begin{array}{l}\text { Filter efficiency (BE). } \\
\text { GIS integration tools } \\
\text { (LR, BE, PE). }\end{array}$ & $\begin{array}{l}\text { Survey of inventory } \\
\text { (LR). Guidance on use } \\
\text { (LR, BE, PE). }\end{array}$ \\
\hline
\end{tabular}

All images are courtesy of Shutterstock. 
TABLE 3-1-5 Support Goal: Survey and Monitoring for Scenario 5

\begin{tabular}{|c|c|c|c|c|c|c|}
\hline Scenario & Summary & Category & \multicolumn{4}{|c|}{ Column Number } \\
\hline \multirow{6}{*}{$\begin{array}{l}\text { Scenario 5: } \\
\text { How would the above } \\
\text { equipment and methods } \\
\text { differ if the contamination } \\
\text { occurred at critical } \\
\text { infrastructure such as a } \\
\text { hospital, wastewater } \\
\text { reclamation facility, } \\
\text { drinking water treatment } \\
\text { plant, airport, or } \\
\text { communication? }\end{array}$} & & & 1 & 2 & 3 & 4 \\
\hline & \multirow{5}{*}{$\begin{array}{l}\text { - Much, if not all, of the discussion on general } \\
\text { surveying from scenarios } 1-4 \text { applies here. } \\
\text { - Hospitals and other critical facilities would } \\
\text { require immediate surveys and gross } \\
\text { decontamination to mitigate doses followed } \\
\text { by either decontamination or fixing of } \\
\text { contamination in place pending future } \\
\text { cleanup. This would allow critical facilities to } \\
\text { continue to operate until the incident had } \\
\text { been stabilized. } \\
\text { - Drinking water treatment plants would } \\
\text { probably be taken offline until full } \\
\text { monitoring and remediation was complete. } \\
\text { These would be priority facilities. They may } \\
\text { require immediate access to portable } \\
\text { filtration/ion exchange plants as part of the } \\
\text { remediation. } \\
\text { - The predicted effect on critical infrastructure } \\
\text { needs to be assessed. Guidance on equipment } \\
\text { use specific to critical infrastructure and GIS } \\
\text { integration will be necessary. }\end{array}$} & EQUIPMENT & Portal monitors. & $\begin{array}{l}\text { Manned, detailed } \\
\text { measurement } \\
\text { surveys. }\end{array}$ & $\begin{array}{l}\text { Radiation speed bump } \\
\text { detectors. }\end{array}$ & Industry air monitors. \\
\hline & & |DESCRIPTION & $\begin{array}{l}\text { Can be used to } \\
\text { segregate contaminated } \\
\text { vehicles at strategic } \\
\text { locations. }\end{array}$ & $\begin{array}{l}\text { Portable instruments } \\
\text { for trained } \\
\text { operators. }\end{array}$ & $\begin{array}{l}\text { Real-time detection to } \\
\text { segregate contaminated } \\
\text { vehicles. }\end{array}$ & $\begin{array}{l}\text { Common nuclear } \\
\text { industry and } \\
\text { laboratory equipment } \\
\text { for continuous } \\
\text { monitoring. Direct or } \\
\text { indirect analysis of air } \\
\text { or filters. }\end{array}$ \\
\hline & & ADVANTAGES & $\begin{array}{l}\text { In use throughout } \\
\text { country to detect } \\
\text { radioactive material or } \\
\text { contamination. Many } \\
\text { designs available for } \\
\text { cars, trucks, and rail } \\
\text { cars. } \\
\end{array}$ & $\begin{array}{l}\text { Large numbers } \\
\text { COTS, mobile. }\end{array}$ & Mobile. & $\begin{array}{l}\text { Nuclear industry } \\
\text { standard. }\end{array}$ \\
\hline & & LIMITATIONS & Limited COTS. & $\begin{array}{l}\text { No GIS integration, } \\
\text { significant } \\
\text { manpower, } \\
\text { background } \\
\text { radiation } \\
\text { interference. }\end{array}$ & $\begin{array}{l}\text { Limited number of } \\
\text { suppliers, gamma only. }\end{array}$ & Cost, filter analysis. \\
\hline & & R\&D NEEDS & $\begin{array}{l}\text { Survey of inventory } \\
\text { (LR). }\end{array}$ & $\begin{array}{l}\text { Guidance on use } \\
\text { specific to critical } \\
\text { infrastructure (LR). } \\
\text { GIS integration } \\
\text { tools (LR, BE, PE). }\end{array}$ & $\begin{array}{l}\text { Guidance on use } \\
\text { specific to critical } \\
\text { infrastructure (LR). } \\
\text { GIS integration tools } \\
\text { (LR, BE, PE). }\end{array}$ & $\begin{array}{l}\text { Guidance on use } \\
\text { specific to critical } \\
\text { infrastructure (LR). } \\
\text { GIS integration tools } \\
\text { (LR, BE, PE). }\end{array}$ \\
\hline
\end{tabular}

All images are personal photographs or courtesy of Shutterstock. 
TABLE 3-1-6 Support Goal: Survey and Monitoring for Scenario 6

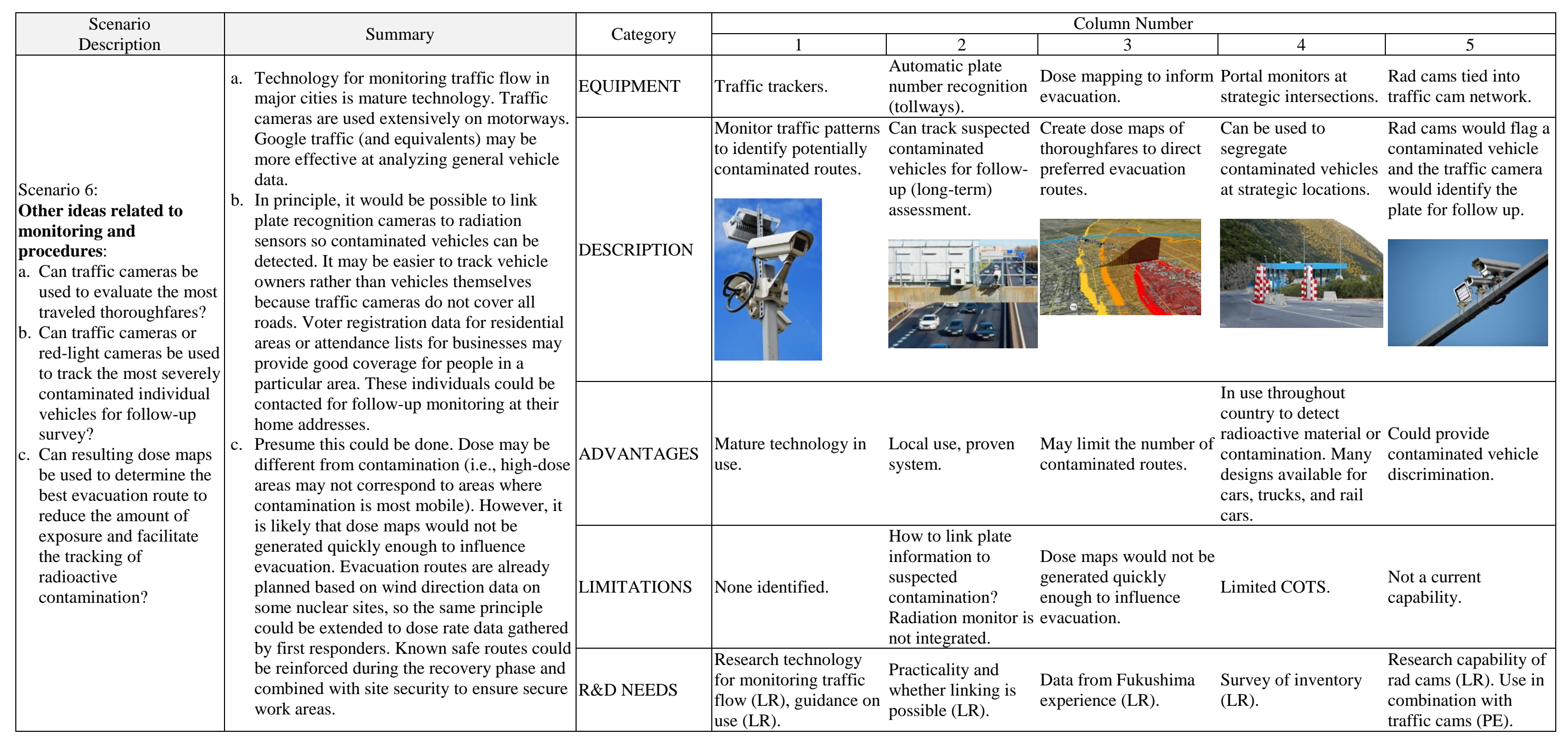

All images are courtesy of Shutterstock, except: (a) B. R. Buddemeier and M. B. Dillon, Key response planning factors for the aftermath of nuclear terrorism, No. LLNL-TR-410067. Lawrence Livermore National Laboratory, Livermore,
CA (United States), 2009. 
TABLE 3-1-7 Support Goal: Survey and Monitoring for Scenario 7

\begin{tabular}{|c|c|c|c|c|c|c|c|c|}
\hline Scenario 7 & \multicolumn{8}{|c|}{ Responses } \\
\hline $\begin{array}{l}\text { Question to participants: } \\
\text { Do you have other } \\
\text { thoughts or specific } \\
\text { questions related to this } \\
\text { topic based on your } \\
\text { experiences in your } \\
\text { geographical area? }\end{array}$ & $\begin{array}{l}\text { The most experienced } \\
\text { existing monitoring } \\
\text { personnel would be best } \\
\text { deployed on: } \\
\text { • High-hazard/high-value } \\
\text { activities (e.g., evidence } \\
\text { recovery), } \\
\text { - Containment activities to } \\
\text { prevent the situation from } \\
\text { degrading further (e.g., } \\
\text { contamination monitoring } \\
\text { at defined boundaries } \\
\text { between areas), } \\
\text { - Training additional } \\
\text { resources to provide } \\
\text { operational monitoring } \\
\text { within the remediation } \\
\text { zones), and } \\
\text { - Clearance monitoring for } \\
\text { priority areas that need to } \\
\text { return to operation quickly. } \\
\text { It would be beneficial to } \\
\text { establish some monitoring } \\
\text { stations where less-trained } \\
\text { staff can use automated } \\
\text { monitoring (such as hand and } \\
\text { foot monitors) with little risk. } \\
\text { These types of stations } \\
\text { already exist at Chernobyl. }\end{array}$ & $\begin{array}{l}\text { There appear to be several } \\
\text { companies that produce } \\
\text { systems for wide-area } \\
\text { monitoring. However, it } \\
\text { is generally not believed } \\
\text { that these would satisfy } \\
\text { all requirements, } \\
\text { specifically: } \\
\text { - Measurement of beta } \\
\text { radiation, } \\
\text { - Common data formats, } \\
\text { - Wireless remote } \\
\text { upload of data. } \\
\text { Therefore, there may be } \\
\text { systems available, but } \\
\text { running their own } \\
\text { custom-made software, } \\
\text { databasese, etc. Difficult/ } \\
\text { time-consuming to import } \\
\text { all data types into one } \\
\text { GIS database. If } \\
\text { authorities had a standard } \\
\text { GIS system setup for such } \\
\text { emergencies, then a } \\
\text { standard data format } \\
\text { could be published to the } \\
\text { supply chain. This would } \\
\text { significantly increase } \\
\text { capability. }\end{array}$ & $\begin{array}{l}\text { Data mapping systems } \\
\text { require significant } \\
\text { effort to set up and } \\
\text { manage, especially if } \\
\text { the area includes large } \\
\text { numbers of complex } \\
\text { structures. The "heat" } \\
\text { of an incident is not } \\
\text { the best time to } \\
\text { determine the } \\
\text { requirements for a } \\
\text { data logging and } \\
\text { visualization system. } \\
\text { This should be } \\
\text { considered a task that } \\
\text { could be, in part, } \\
\text { completed prior to any } \\
\text { incident. }\end{array}$ & $\begin{array}{l}\text { Data recording within } \\
\text { buildings could, in } \\
\text { theory, be performed } \\
\text { by installed monitors } \\
\text { that report readings } \\
\text { automatically. For } \\
\text { larger buildings and } \\
\text { businesses that have } \\
\text { air conditioning, the } \\
\text { air inlets/filters could } \\
\text { be monitored. Such } \\
\text { data recording systems } \\
\text { are used within } \\
\text { nuclear plants } \\
\text { throughout the world. }\end{array}$ & $\begin{array}{l}\text { In practice, there would } \\
\text { not be enough time, } \\
\text { money, or systems } \\
\text { available to set up such a } \\
\text { comprehensive system. In } \\
\text { the short to medium term, } \\
\text { the easiest system to set } \\
\text { up would be to provide } \\
\text { residences (or groups of } \\
\text { residence) monitoring } \\
\text { equipment and ask for } \\
\text { readings to be supplied } \\
\text { regularly. The training is } \\
\text { minimal and is only } \\
\text { reliant on enough suitable } \\
\text { pieces of equipment being } \\
\text { available. There is a wide } \\
\text { variety of equipment } \\
\text { available from the supply, } \\
\text { which all provide gamma } \\
\text { dose rate. }\end{array}$ & $\begin{array}{l}\text { Air samplers } \\
\text { Question: is/are there } \\
\text { enough: } \\
\text { - Suitable systems } \\
\text { available? } \\
\text { - Personnel to install and } \\
\text { maintain them? } \\
\text { - Money available? } \\
\text { - Need/desire to do this? }\end{array}$ & $\begin{array}{l}\text { Guidance on use and GIS } \\
\text { integration of the } \\
\text { equipment listed will be } \\
\text { necessary. }\end{array}$ & $\begin{array}{l}\text { A few things that must be kept } \\
\text { in mind are that: 1) Different } \\
\text { detectors serve different } \\
\text { purposes and have different } \\
\text { capabilities. For instance, some } \\
\text { are GPS-enabled; some } \\
\text { detectors saturate at relatively } \\
\text { low levels of contamination; } \\
\text { detectors provide different } \\
\text { outputs as some are dose } \\
\text { meters, spectrometers, or } \\
\text { exposure meters; and some } \\
\text { detectors transmit data } \\
\text { automatically, but the vast } \\
\text { majority do not. 2). The goal of } \\
\text { the mapping must be explained. } \\
\text { Is it to provide information to } \\
\text { the public or to characterize a } \\
\text { surface for decontamination? }\end{array}$ \\
\hline
\end{tabular}


TABLE 3-1-8 Support Goal Training: Survey and Monitoring for Scenarios 1-7

\begin{tabular}{|c|c|c|c|c|c|c|c|c|c|}
\hline $\begin{array}{l}\text { Support Goal Training: } \\
\text { Scenarios 1-7 }\end{array}$ & Summary & & & & Respons & nses & & & \\
\hline \begin{tabular}{|l} 
Question to participants: \\
What are your thoughts and \\
recommendations on \\
availability of trained \\
human assets and training \\
of additional assets that will \\
likely be needed in order to \\
accomplish the scenarios \\
under this goal? It is \\
understood that training will \\
be a significant effort and an \\
additional limiting factor in \\
any response scenario. \\
Further, it may need to be \\
addressed more thoroughly \\
in the future, but input is \\
needed to help guide how \\
training guidance should be \\
developed.
\end{tabular} & $\begin{array}{l}\text { - Wide-area radiological } \\
\text { contamination incidents are } \\
\text { rare, and a response to such } \\
\text { an incident will require } \\
\text { tremendous human assets. } \\
\text { Lessons learned from the } \\
\text { cleanup efforts in Japan } \\
\text { show that many thousands } \\
\text { of individuals each day are } \\
\text { engaged in cleanup } \\
\text { activities. } \\
\text { - For smaller incidents, all } \\
\text { surveying and monitoring } \\
\text { work would be completed } \\
\text { by trained health physicist } \\
\text { (HP) monitors with } \\
\text { radiation instrumentation. } \\
\text { During a larger incident, } \\
\text { there may be a requirement } \\
\text { to ask members of the } \\
\text { public to perform basic } \\
\text { surveying, potentially using } \\
\text { smart-phone capabilities. }\end{array}$ & $\begin{array}{l}\text { There is a limited number of } \\
\text { suitably qualified and } \\
\text { experienced persons (SQEPs) } \\
\text { and companies that offer } \\
\text { proper training. Sections of a } \\
\text { typical national vocational } \\
\text { qualification (NVQ) syllabus } \\
\text { could be extracted to meet the } \\
\text { requirements of different } \\
\text { roles. A general approach to } \\
\text { grade the level of training } \\
\text { required could be considered, } \\
\text { such as those listed in the next } \\
\text { cells. }\end{array}$ & $\begin{array}{l}\text { Level 1: Low-risk } \\
\text { activities (e.g., operating a } \\
\text { monitoring vehicle while } \\
\text { remaining in a sealed cab } \\
\text { at all times). Personnel } \\
\text { would have a basic } \\
\text { understanding of } \\
\text { radiological risk and } \\
\text { understand the dress, } \\
\text { undress, and monitoring } \\
\text { procedures for their role. }\end{array}$ & $\begin{array}{l}\text { Level 2: Low-risk } \\
\text { activities such as } \\
\text { monitoring items and } \\
\text { personnel moving } \\
\text { between relatively } \\
\text { clean areas (such as } \\
\text { from sealed vehicle } \\
\text { cabs at designated } \\
\text { clean areas). }\end{array}$ & \begin{tabular}{l|l} 
Level 3: These would \\
be people with \\
baseline training as \\
noted in Levels 1 and \\
2 , and some \\
experience, who have \\
demonstrated \\
competence in their \\
technical field.
\end{tabular} & $\begin{array}{l}\text { Level 4: These would } \\
\text { be people who have } \\
\text { more experience and a } \\
\text { broader knowledge of } \\
\text { the operations and } \\
\text { required controls. }\end{array}$ & $\begin{array}{l}\text { Level 5: These are } \\
\text { fully competent and } \\
\text { experienced } \\
\text { personnel who could } \\
\text { lead and mentor } \\
\text { lower-level } \\
\text { personnel. }\end{array}$ & \begin{tabular}{|l|} 
Provision of basic \\
radiation awareness \\
training can be web- \\
(e-learning) or \\
classroom-based \\
learning. There will be \\
a requirement for \\
practical sessions and \\
supervision for all \\
persons who are new to \\
radiological hazards.
\end{tabular} & $\begin{array}{l}\text { Persons who have a basic } \\
\text { understanding of radiation and } \\
\text { contamination (nuclear } \\
\text { facilities, non-nuclear } \\
\text { facilities, hospitals, } \\
\text { emergency services, and some } \\
\text { military personnel), or have an } \\
\text { understanding of other } \\
\text { hazards (asbestos or } \\
\text { chemicals) that can be applied } \\
\text { to radiation/contamination } \\
\text { hazard. }\end{array}$ \\
\hline
\end{tabular}




\subsection{SUPPORT GOAL: MITIGATION OF RECEIVED DOSE TO FIRST RESPONDERS}

Radiation dose burden for response personnel is a concern and must be reduced. What types of municipal and commercial equipment can carry out gross decontamination of contaminated surfaces and can contain and prevent the resuspension and tracking of contamination - either through the effects of wind or vehicle transport - during the mitigation phase of the response to reduce the dose burden to first-responder teams? Examples include using fireboats from a Port Authority to knock down radioactivity levels near the shore or dump trucks and bobcats to spread mulch and gravel across roadways to reduce the spread of contamination during vehicle transport. Note that "gross decontamination" is a type of decontamination whose goal is to reduce contamination levels. This reduction may not reach final cleanup levels, but it may be useful to mitigate some public hazard or contain contamination. Tables 3-2-1 to 3-2-10 present various scenarios related to mitigation of the received dose to first responders, possible responses to the scenarios, and possible equipment/technology used in responding, including its advantages, limitations, and R\&D needs. 
TABLE 3-2-1 Support Goal: Mitigation of Received Dose to First Responders for Scenario 1

\begin{tabular}{|c|c|c|c|c|c|c|c|c|}
\hline Scenario Description & Summary & Category & \multicolumn{6}{|c|}{ Column Number } \\
\hline \multirow{6}{*}{$\begin{array}{l}\text { Scenario 1: } \\
\text { Airborne contamination is a } \\
\text { significant source of radiation } \\
\text { dose to unprotected people. } \\
\text { Given that contamination after } \\
\text { settling is primarily found on } \\
\text { horizontal surfaces (e.g., } \\
\text { streets, walkways, parking lots, } \\
\text { urban green spaces), reducing } \\
\text { the potential for resuspension is } \\
\text { potentially important. Prior } \\
\text { research identified materials } \\
\text { such as gravel, mulch, and } \\
\text { sand as effective means of } \\
\text { reducing resuspension if the } \\
\text { material can be laid down } \\
\text { over the contaminated } \\
\text { surface. How would such } \\
\text { material be distributed over an } \\
\text { area of several linear blocks? } \\
\text { How would such material be } \\
\text { distributed over an area of } \\
\text { several square miles? }\end{array}$} & & Caltegiy & 1 & 2 & 3 & 4 & 5 & 6 \\
\hline & \multirow{5}{*}{$\begin{array}{l}\text { - All methods that add material } \\
\text { (e.g., gravel, mulch, sand) } \\
\text { may increase the volume of } \\
\text { radioactive waste. } \\
\text { - Several kinds of equipment } \\
\text { identified will be impractical } \\
\text { in terms of distributing or } \\
\text { spreading the quantity needed. } \\
\text { Dump trucks can handle large } \\
\text { capacities, but will require } \\
\text { additional equipment to } \\
\text { spread the material. } \\
\text { Information gathering will be } \\
\text { necessary to determine } \\
\text { whether the listede equipment } \\
\text { is capable of distributing or } \\
\text { spreading materials such as } \\
\text { gravel, mulch, and sand. } \\
\text { - A common unresolved issue is } \\
\text { that mechanical equipment } \\
\text { used for this work is not } \\
\text { typically supplied with sealed } \\
\text { cabs and HEPA filtration. } \\
\text { Therefore, operator PPE needs } \\
\text { to be addressed and } \\
\text { equipment may need to be } \\
\text { modified prior to use. }\end{array}$} & EQUIPMENT & $\begin{array}{l}\begin{array}{l}\text { Small drop } \\
\text { spreader. }\end{array} \\
\end{array}$ & $\begin{array}{l}\text { Medium salt } \\
\text { spreader. }\end{array}$ & Large salt spreader. & Large chip spreader. & Dumper truck. & Track-type tractor. \\
\hline & & DESCRIPTION & \begin{tabular}{|l} 
Used to spread \\
small particles \\
(fertilizer, sand, \\
seed, salt) on \\
small areas.
\end{tabular} & $\begin{array}{l}\text { Used to spread } \\
\text { small to medium } \\
\text { particles (sand, salt, } \\
\text { gravel) on } \\
\text { roadways and } \\
\text { parking lots. }\end{array}$ & $\begin{array}{l}\text { Used to spread small } \\
\text { to medium particles } \\
\text { (sand, salt, gravel) } \\
\text { on roadways and } \\
\text { parking lots. }\end{array}$ & $\begin{array}{l}\text { Used to spread small } \\
\text { to large gravel to } \\
\text { repave roadways and } \\
\text { parking lots. }\end{array}$ & $\begin{array}{l}\text { Capable of } \\
\text { dumping all types } \\
\text { of materials. }\end{array}$ & $\begin{array}{l}\text { Used to spread mulch } \\
\text { and potentially other } \\
\text { materials. }\end{array}$ \\
\hline & & ADVANTAGES & $\begin{array}{l}\text { Able to treat small } \\
\text { or poorly } \\
\text { accessible areas. } \\
\text { Large number } \\
\text { COTS. }\end{array}$ & $\begin{array}{l}\text { Attached to pickup } \\
\text { truck. Large } \\
\text { numbers COTS in } \\
\text { cold climates. }\end{array}$ & $\begin{array}{l}\text { Fully automated } \\
\text { with wet/dry } \\
\text { combination } \\
\text { spraying/spreading. } \\
\text { Large numbers } \\
\text { COTS in cold } \\
\text { climates. }\end{array}$ & $\begin{array}{l}\text { Large rate of coverage, } \\
\text { fully automated with } \\
\text { conveyors to spread } \\
\text { aggregate. }\end{array}$ & $\begin{array}{l}\text { All sizes available, } \\
\text { large quantities } \\
\text { available, large } \\
\text { capacity, COTS. }\end{array}$ & $\begin{array}{l}\text { COTS, availability. } \\
\text { Could be used with a } \\
\text { dumper truck. }\end{array}$ \\
\hline & & LIMITATIONS & $\begin{array}{l}\text { The coverage rate } \\
\text { of material may } \\
\text { not be sufficient } \\
\text { for the goal. }\end{array}$ & $\begin{array}{l}\text { The rate of } \\
\text { coverage/ } \\
\text { dispersion rate may } \\
\text { not be sufficient for } \\
\text { the goal. }\end{array}$ & $\begin{array}{l}\text { Rate of coverage/ } \\
\text { dispersion may not } \\
\text { be sufficient for the } \\
\text { goal. }\end{array}$ & Availability. & $\begin{array}{l}\text { Gross spreader. } \\
\text { Dumps. Does not } \\
\text { spread. }\end{array}$ & $\begin{array}{l}\text { Coverage is limited. } \\
\text { Large quantities may } \\
\text { be needed. }\end{array}$ \\
\hline & & R\&D NEEDS & $\begin{array}{l}\text { Dispersion rate } \\
\text { (LR). }\end{array}$ & $\begin{array}{l}\text { Compatibility with } \\
\text { material other than } \\
\text { salt (LR). } \\
\text { Dispersion rate } \\
\text { (LR). }\end{array}$ & $\begin{array}{l}\text { Compatibility with } \\
\text { material other than } \\
\text { salt (LR). } \\
\text { Dispersion rate } \\
\text { (LR). }\end{array}$ & $\begin{array}{l}\text { Survey of inventory } \\
\text { (local, regional, } \\
\text { national, private, } \\
\text { commercial) (LR). } \\
\text { Best practice guidance } \\
\text { (LR). }\end{array}$ & $\begin{array}{l}\text { Effective ways to } \\
\text { spread dumped } \\
\text { material (LR). }\end{array}$ & $\begin{array}{l}\text { Survey of inventory } \\
\text { (LR). }\end{array}$ \\
\hline
\end{tabular}


TABLE 3-2-2 Support Goal: Mitigation of Received Dose to First Responders for Scenario 2

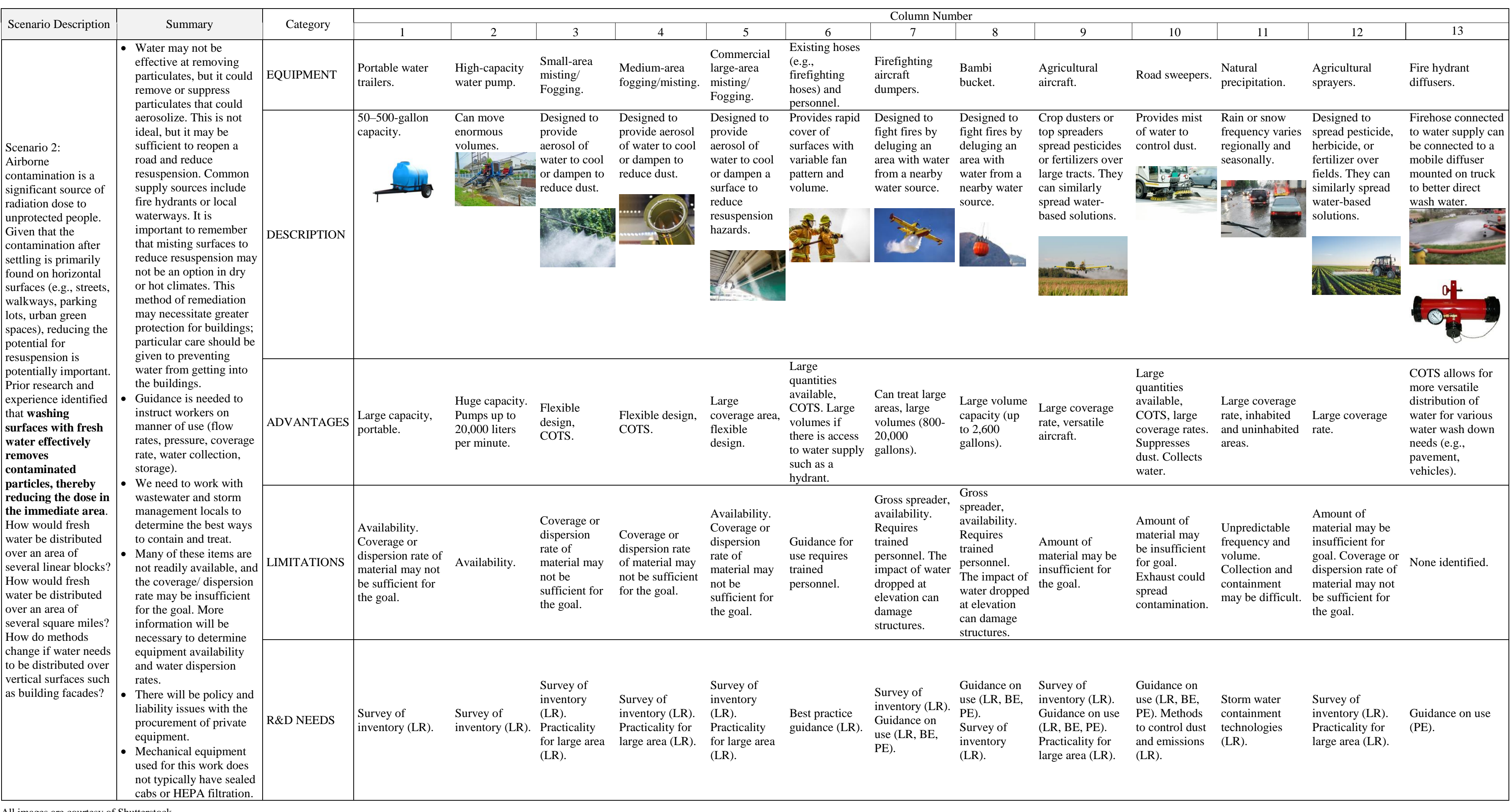


TABLE 3-2-3 Support Goal: Mitigation of Received Dose to First Responders for Scenario 3

\begin{tabular}{|c|c|c|c|c|c|c|c|c|c|c|c|c|c|c|c|}
\hline \multirow{2}{*}{ Scenario Description } & \multirow{2}{*}{ Summary } & \multirow{2}{*}{ Category } & \multicolumn{13}{|c|}{ Column Number } \\
\hline & & & 1 & 2 & 3 & 4 & 5 & 6 & & 8 & 9 & 10 & 11 & 12 & 13 \\
\hline \multirow{5}{*}{ 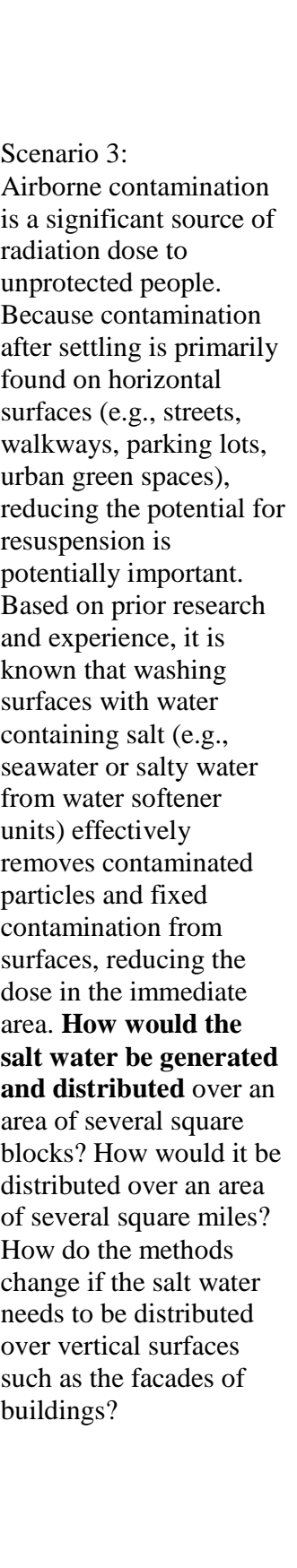 } & \multirow{5}{*}{$\begin{array}{l}\text { - EPA studies have shown } \\
\text { that salt is notg good for } \\
\text { shielding radiation, but it } \\
\text { is a good suppressant } \\
\text { and will reduce } \\
\text { resuspension. } \\
\text { - Salt could be spread in a } \\
\text { solid form, and rain } \\
\text { allowed to mix with it to } \\
\text { make a solution. It was } \\
\text { agreed that a salt-water } \\
\text { solution is more } \\
\text { effective than rain alone. } \\
\text { If spreading a solution, a } \\
\text { cost analyses would need } \\
\text { to be done to compare } \\
\text { seawater ss. onsite } \\
\text { mixing. Participants } \\
\text { referenced a studdy that } \\
\text { showed dry sults to have } \\
\text { no effect on fertility of } \\
\text { soil. However, when a } \\
\text { saline solution was } \\
\text { applied, it killed the } \\
\text { vegegetation and caused } \\
\text { more dust to aerosolize } \\
\text { from barren soil. } \\
\text { - As in Scenario } 2 \text {, pumps } \\
\text { and misting equipment } \\
\text { can be used with } \\
\text { seawater/salty water, } \\
\text { although corrosion may } \\
\text { affect long-term lifespan. } \\
\text { The same concerns over } \\
\text { proper use, coverage } \\
\text { rates, water containment, } \\
\text { worker protection apply. }\end{array}$} & EQUIPMENT & $\begin{array}{l}\text { Intermediate } \\
\text { bulk container } \\
\text { (IBC) tote } \\
\text { mixer. }\end{array}$ & $\begin{array}{l}\text { IBC pump and } \\
\text { sprayer. }\end{array}$ & $\begin{array}{l}\text { Existing hoses } \\
\text { (e.g., } \\
\text { firefighting } \\
\text { hoses) and } \\
\text { personnel. }\end{array}$ & $\begin{array}{l}\text { Firefighting } \\
\text { aircraft } \\
\text { dumpers. }\end{array}$ & Bambi bucket. & $\begin{array}{l}\text { Agricultural } \\
\text { aircraft. }\end{array}$ & $\begin{array}{l}\text { Portable water } \\
\text { trailers. }\end{array}$ & $\begin{array}{l}\text { High capacity } \\
\text { water pump. }\end{array}$ & $\begin{array}{l}\text { mall area } \\
\text { isting/ } \\
\text { sging. }\end{array}$ & $\begin{array}{l}\text { Medium area } \\
\text { fogging/ } \\
\text { Misting. }\end{array}$ & $\begin{array}{l}\text { Commercial } \\
\text { large area } \\
\text { misting/ } \\
\text { Fogging. }\end{array}$ & Road sweepers. & $\begin{array}{l}\text { Stackpiled rock } \\
\text { salt for deicing. }\end{array}$ \\
\hline & & DESCRIPTION & $\begin{array}{l}\begin{array}{l}\text { Diverse } \\
\text { category of } \\
\text { industrial } \\
\text { mixers } \\
\text { designed to } \\
\text { meet various } \\
\text { needs for } \\
\text { agitation in } \\
\text { these } \\
\text { containers. }\end{array} \\
\end{array}$ & $\begin{array}{l}\text { https://www.as } \\
\text { phaltsealcoatin } \\
\text { gdirect.com/pr } \\
\text { oducts/asd275- } \\
\text { portable- } \\
\text { sealcoating- } \\
\text { spray-system } \\
\end{array}$ & $\begin{array}{l}\text { Provide rapid } \\
\text { cover of } \\
\text { surfaces with } \\
\text { variable fan } \\
\text { pattern and } \\
\text { volume. }\end{array}$ & $\begin{array}{l}\text { Designed to } \\
\text { fight fires by } \\
\text { deluging area } \\
\text { with water } \\
\text { from nearby } \\
\text { water source. }\end{array}$ & $\begin{array}{l}\text { Designed to } \\
\text { fight fires by } \\
\text { deluging an } \\
\text { area with } \\
\text { water from a } \\
\text { nearby water } \\
\text { source. }\end{array}$ & $\begin{array}{l}\text { Crop dusters or } \\
\text { top spreaders } \\
\text { spread } \\
\text { pesticides or } \\
\text { fertilizers over } \\
\text { large tracts. } \\
\text { They can } \\
\text { similarly } \\
\text { spread water- } \\
\text { based } \\
\text { solutions. }\end{array}$ & $\begin{array}{l}\text { 50-500-gallon } \\
\text { capacity. }\end{array}$ & $\begin{array}{l}\text { Can pump } \\
\text { water at rates } \\
\text { of up to } \\
1000 \mathrm{~m}^{3} / \mathrm{h} \text {. }\end{array}$ & $\begin{array}{l}\text { Designed to } \\
\text { provide aerosol } \\
\text { of water to cool } \\
\text { or dampen to } \\
\text { reduce dust. }\end{array}$ & $\begin{array}{l}\text { Designed to } \\
\text { provide aerosol } \\
\text { of water to cool } \\
\text { or dampen to } \\
\text { reduce dust. }\end{array}$ & $\begin{array}{l}\text { Designed to } \\
\text { provide aerosol } \\
\text { of water to cool } \\
\text { or dampen to } \\
\text { reduce dust. }\end{array}$ & $\begin{array}{l}\text { Provides mist } \\
\text { of water to } \\
\text { control dust. }\end{array}$ & $\begin{array}{l}\text { Sodium-based } \\
\text { deicing salt can } \\
\text { be used to create } \\
\text { effective wash } \\
\text { solution to } \\
\text { improve de- } \\
\text { contamination. }\end{array}$ \\
\hline & & ADVANTAGES & \begin{tabular}{|l} 
Up to \\
250-gallon \\
capacity, large \\
quantities \\
available \\
COTS.
\end{tabular} & $\begin{array}{l}\text { Hand or } \\
\text { electric pump, } \\
\text { large quantities } \\
\text { available } \\
\text { COTS. }\end{array}$ & $\begin{array}{l}\text { Large } \\
\text { quantities } \\
\text { available } \\
\text { COTS. }\end{array}$ & $\begin{array}{l}\text { an treat } \\
\text { rge areas, } \\
\text { rge volumes } \\
300-20,000 \\
\text { allons). }\end{array}$ & $\begin{array}{l}\text { Large volume } \\
\text { capacity (up } \\
\text { to } 2600 \\
\text { gallons). }\end{array}$ & $\begin{array}{l}\text { Large coverage } \\
\text { rates. }\end{array}$ & $\begin{array}{l}\text { Large capacity, } \\
\text { portable. }\end{array}$ & $\begin{array}{l}\text { Huge } \\
\text { capacity. }\end{array}$ & $\begin{array}{l}\text { Flexible } \\
\text { design, COTS. }\end{array}$ & $\begin{array}{l}\text { Flexible } \\
\text { design, COTS. }\end{array}$ & $\begin{array}{l}\text { Large coverage } \\
\text { area, flexible } \\
\text { design. }\end{array}$ & $\begin{array}{l}\text { Large coverage } \\
\text { rates. } \\
\text { Suppresses } \\
\text { dust. Collects } \\
\text { water. }\end{array}$ & $\begin{array}{l}\text { Common in } \\
\text { northern U.S. } \\
\text { cities, large } \\
\text { stockpiles, } \\
\text { existing supply } \\
\text { chain. }\end{array}$ \\
\hline & & IMITATIONS & $\begin{array}{l}\text { None } \\
\text { identified. }\end{array}$ & $\begin{array}{l}\text { None } \\
\text { identified. }\end{array}$ & $\begin{array}{l}\text { Salt supply } \\
\text { needs to be } \\
\text { integrated into } \\
\text { spray system. } \\
\text { Incompatible } \\
\text { with salt. }\end{array}$ & $\begin{array}{l}\text { Gross } \\
\text { spreader, } \\
\text { limited } \\
\text { availability. } \\
\text { Requires } \\
\text { trained } \\
\text { personnel. } \\
\text { The impact of } \\
\text { water dropped } \\
\text { at elevation } \\
\text { can damage } \\
\text { structures. }\end{array}$ & $\begin{array}{l}\text { Gross } \\
\text { spreader, } \\
\text { availability. } \\
\text { Requires } \\
\text { trained } \\
\text { personnel. } \\
\text { The impact of } \\
\text { water dropped } \\
\text { at elevation } \\
\text { can damage } \\
\text { structures. }\end{array}$ & $\begin{array}{l}\text { Amount of } \\
\text { material may } \\
\text { be insufficient } \\
\text { for goal. } \\
\text { Incompatibility } \\
\text { with salt. }\end{array}$ & $\begin{array}{l}\text { Availability, } \\
\text { incompatible } \\
\text { with salt. } \\
\text { Coverage or } \\
\text { dispersion rate } \\
\text { of material may } \\
\text { not be } \\
\text { sufficient for } \\
\text { the goal. }\end{array}$ & $\begin{array}{l}\text { Availability, } \\
\text { incompatible } \\
\text { with salt. }\end{array}$ & $\begin{array}{l}\text { Coverage or } \\
\text { dispersion rate } \\
\text { of material may } \\
\text { not be } \\
\text { sufficient for } \\
\text { the goal. } \\
\text { Incompatible } \\
\text { with salt. }\end{array}$ & $\begin{array}{l}\text { Coverage or } \\
\text { dispersion rate } \\
\text { of material may } \\
\text { not be } \\
\text { sufficient for } \\
\text { the goal. } \\
\text { Incompatible } \\
\text { with salt. }\end{array}$ & $\begin{array}{l}\text { Availability, } \\
\text { incompatible } \\
\text { with salt. } \\
\text { Coverage or } \\
\text { dispersion rate } \\
\text { of material may } \\
\text { not be } \\
\text { sufficient for } \\
\text { the goal. }\end{array}$ & $\begin{array}{l}\text { Amount of } \\
\text { material may } \\
\text { be insufficient } \\
\text { for goal. } \\
\text { Exhaust could } \\
\text { spread } \\
\text { contamination. } \\
\text { Incompatible } \\
\text { with salt. }\end{array}$ & $\begin{array}{l}\text { Produc } \\
\text { uncom } \\
\text { southe }\end{array}$ \\
\hline & & R\&D NEEDS & $\begin{array}{l}\text { None } \\
\text { identified. }\end{array}$ & $\begin{array}{l}\text { None } \\
\text { identified. }\end{array}$ & $\begin{array}{l}\text { Guidance on } \\
\text { effect of } \\
\text { different salts } \\
\text { and } \\
\text { concentrations } \\
\text { (LR, BE, PE). } \\
\text { Compatibility } \\
\text { with salt (LR). }\end{array}$ & $\begin{array}{l}\text { Survey of } \\
\text { inventory } \\
\text { (LR). } \\
\text { Guidance on } \\
\text { use (LR, BE, } \\
\text { PE). }\end{array}$ & $\begin{array}{l}\text { Survey of } \\
\text { inventory } \\
\text { (LR). } \\
\text { Guidance on } \\
\text { use (LR, BE, } \\
\text { PE). }\end{array}$ & $\begin{array}{l}\text { Survey of } \\
\text { inventory (LR). } \\
\text { Compatibility } \\
\text { with salt (LR). }\end{array}$ & $\begin{array}{l}\text { Survey of } \\
\text { inventory (LR). } \\
\text { Compatibility } \\
\text { with salt (LR). }\end{array}$ & $\begin{array}{l}\text { Survey of } \\
\text { inventory } \\
\text { (LR). } \\
\text { Compatibility } \\
\text { with salt } \\
\text { (LR). }\end{array}$ & $\begin{array}{l}\text { Survey of } \\
\text { inventory (LR). } \\
\text { Compatibility } \\
\text { with salt (LR). } \\
\text { Practicality for } \\
\text { large area } \\
\text { (LR). }\end{array}$ & $\begin{array}{l}\text { Survey of } \\
\text { inventory (LR). } \\
\text { Compatibility } \\
\text { with salt (LR). } \\
\text { Practicality for } \\
\text { large area } \\
\text { (LR). }\end{array}$ & $\begin{array}{l}\text { Survey of } \\
\text { inventory (LR). } \\
\text { Compatibility } \\
\text { with salt (LR). } \\
\text { Practicality for } \\
\text { large area } \\
\text { (LR). }\end{array}$ & $\begin{array}{l}\text { Guidance on } \\
\text { use (LR, BE, } \\
\text { PE). } \\
\text { Compatibility } \\
\text { with salt (LR). } \\
\text { Methods to } \\
\text { control dust } \\
\text { and emissions } \\
\text { (LR). }\end{array}$ & $\begin{array}{l}\text { Research salt } \\
\text { varieties } \\
\text { recommended } \\
\text { for use (LR). }\end{array}$ \\
\hline
\end{tabular}


TABLE 3-2-3 (Cont.)

\begin{tabular}{|c|c|c|c|c|c|c|c|c|}
\hline & \multirow{2}{*}{ Summary } & \multirow{2}{*}{ Category } & \multicolumn{5}{|c|}{ Column Number } & \multirow[b]{2}{*}{19} \\
\hline & & & 14 & 15 & 16 & 17 & 18 & \\
\hline \multirow{5}{*}{ Scenario Description } & & EQUIPMENT & $\begin{array}{l}\text { Agricultural } \\
\text { sprayers. }\end{array}$ & $\begin{array}{l}\text { Small drop } \\
\text { spreader. }\end{array}$ & $\begin{array}{l}\text { Medium salt } \\
\text { spreader. }\end{array}$ & $\begin{array}{l}\text { Large salt } \\
\text { spreader. }\end{array}$ & Paver trucks. & Fire hydrant diffusers. \\
\hline & & DESCRIPTION & $\begin{array}{l}\text { Designed for } \\
\text { distributing } \\
\text { pesticide, } \\
\text { herbicide, or } \\
\text { fertilizer on fields. } \\
\text { They can similarly } \\
\text { spread water-based } \\
\text { solutions. }\end{array}$ & $\begin{array}{l}\text { Used to spread } \\
\text { small particles } \\
\text { (fertilizer, } \\
\text { sand, seed, salt) } \\
\text { over small } \\
\text { areas. }\end{array}$ & $\begin{array}{l}\text { Used to spread } \\
\text { small to medium } \\
\text { particles (sand, } \\
\text { salt, gravel) on } \\
\text { roadways and } \\
\text { parking lots. }\end{array}$ & $\begin{array}{l}\text { Used to spread } \\
\text { small to medium } \\
\text { particles (sand, } \\
\text { salt, gravel) on } \\
\text { roadways and } \\
\text { parking lots. }\end{array}$ & $\begin{array}{l}\text { Covers single } \\
\text { lane of roadway } \\
\text { in one pass with } \\
\text { variable depth of } \\
\text { asphalt. }\end{array}$ & $\begin{array}{l}\text { Firehose connected to } \\
\text { salt water supply can } \\
\text { be connected to a } \\
\text { mobile diffuser } \\
\text { mounted on truck to } \\
\text { better direct wash } \\
\text { water. }\end{array}$ \\
\hline & & ADVANTAGES & $\begin{array}{l}\text { Large coverage } \\
\text { rate. }\end{array}$ & $\begin{array}{l}\text { Can treat small } \\
\text { or poorly } \\
\text { accessible } \\
\text { areas. Large } \\
\text { number COTS. }\end{array}$ & $\begin{array}{l}\text { Attached to } \\
\text { pickups. Large } \\
\text { number COTS in } \\
\text { cold climates. }\end{array}$ & $\begin{array}{l}\text { Fully automated } \\
\text { with wet/dry } \\
\text { combination } \\
\text { spraying/ } \\
\text { spreading. Large } \\
\text { number COTS in } \\
\text { cold climates. }\end{array}$ & $\begin{array}{l}\text { Large quantities } \\
\text { available COTS. }\end{array}$ & $\begin{array}{l}\text { COTS allows for more } \\
\text { versatile distribution of } \\
\text { water for various water } \\
\text { wash down needs (e.g., } \\
\text { pavement, vehicles). }\end{array}$ \\
\hline & & LIMITATIONS & $\begin{array}{l}\text { Coverage or } \\
\text { dispersion rate of } \\
\text { material may be } \\
\text { insufficient for the } \\
\text { goal. Compatibility } \\
\text { with salt. }\end{array}$ & $\begin{array}{l}\text { Coverage or } \\
\text { dispersion rate } \\
\text { of material may } \\
\text { not be } \\
\text { sufficient for } \\
\text { the goal. }\end{array}$ & $\begin{array}{l}\text { Rate of coverage/ } \\
\text { dispersion may be } \\
\text { insufficient for } \\
\text { goal. }\end{array}$ & $\begin{array}{l}\text { Rate of coverage/ } \\
\text { dispersion may be } \\
\text { insufficient for } \\
\text { goal. }\end{array}$ & $\begin{array}{l}\text { Incompatible with } \\
\text { salt. }\end{array}$ & None identified. \\
\hline & & R\&D NEEDS & $\begin{array}{l}\text { Survey of } \\
\text { inventory (LR). } \\
\text { Compatibility with } \\
\text { salt (LR). } \\
\text { Practicality for } \\
\text { large area (LR). } \\
\text { Guidance on use } \\
\text { (LR, BE, PE). }\end{array}$ & $\begin{array}{l}\text { None } \\
\text { identified. }\end{array}$ & $\begin{array}{l}\text { Dispersion rate } \\
\text { (LR). Guidance } \\
\text { on use (LR, BE, } \\
\text { PE). }\end{array}$ & $\begin{array}{l}\text { Dispersion rate } \\
\text { (LR). Guidance on } \\
\text { use (LR, BE, PE). }\end{array}$ & $\begin{array}{l}\text { Compatibility } \\
\text { with salt (LR). }\end{array}$ & Guidance on use (PE). \\
\hline
\end{tabular}

All images are courtesy of Shutterstock. 
TABLE 3-2-4 Support Goal: Mitigation of Received Dose to First Responders for Scenario 4

\begin{tabular}{|c|c|c|c|c|c|c|c|c|c|}
\hline \multirow{2}{*}{$\begin{array}{c}\text { Scenario } \\
\text { Description }\end{array}$} & \multirow{2}{*}{ Summary } & \multirow{2}{*}{ Category } & \multicolumn{7}{|c|}{ Column Number } \\
\hline & & & 1 & 2 & 3 & 4 & 5 & 6 & 7 \\
\hline \multirow{5}{*}{$\begin{array}{l}\text { Scenario 4: } \\
\text { How would the proposed methods } \\
\text { of addressing Scenarios } 1-3 \\
\text { change if the contaminated area } \\
\text { was located close enough to the } \\
\text { ocean coastline or another } \\
\text { navigable waterway for maritime } \\
\text { equipment and methods to be } \\
\text { used? }\end{array}$} & \multirow{5}{*}{$\begin{array}{l}\text { Waterborne equipment could } \\
\text { draw water from the waterway } \\
\text { and provide floating } \\
\text { emergency accommodations. } \\
\text { - Fireboats charge water lines to } \\
\text { clean bridges with river and/or } \\
\text { seawater. They could } \\
\text { potentially decontaminate } \\
\text { bridges and cars. } \\
\text { - Standard personal, municipal, } \\
\text { or } \\
\text { commercial watercraft can also } \\
\text { be used if pumps and hoses are } \\
\text { added to the craft. The easy } \\
\text { addition of pumps to normal } \\
\text { craft would allow them to draw } \\
\text { water directly from the ocean } \\
\text { or waterway and pump it } \\
\text { straight onto buildings. } \\
\text { Many of the items listed are } \\
\text { not readily available or are } \\
\text { limited to immediate coastal } \\
\text { coverage. } \\
\text { There will be policy and } \\
\text { liability issues with the } \\
\text { procurement of private boats. } \\
\text { Equipment is not designed for } \\
\text { contaminated environments, } \\
\text { and operator PPE needs to be } \\
\text { addressed (estimate of the dose } \\
\text { to workers is not established, } \\
\text { estimated, or summarized). }\end{array}$} & EQUIPMENT & $\begin{array}{l}\text { High-capacity water } \\
\text { pump. }\end{array}$ & Fireboats. & $\begin{array}{l}\text { Personal, municipal, } \\
\text { and commercial } \\
\text { watercraft. }\end{array}$ & Cruise/cargo ship. & Sand. & $\begin{array}{l}\text { Fireboats and bridge } \\
\text { decontamination. }\end{array}$ & Fire hydrant diffusers. \\
\hline & & DESCRIPTION & $\begin{array}{l}\text { Can move enormous } \\
\text { volumes of water. }\end{array}$ & $\begin{array}{l}\text { Designed for } \\
\text { structures in or } \\
\text { adjacent to waterway, } \\
\text { but can supply water } \\
\text { throughout city via } \\
\text { piping. }\end{array}$ & $\begin{array}{l}\text { Personal, municipal, } \\
\text { or commercial } \\
\text { watercraft can be } \\
\text { used if pumps and } \\
\text { hoses are added to the } \\
\text { craft. }\end{array}$ & $\begin{array}{l}\text { Accommodation for } \\
\text { workers. }\end{array}$ & $\begin{array}{l}\text { Can cover contaminated } \\
\text { fallout. }\end{array}$ & $\begin{array}{l}\text { Can decontaminate } \\
\text { bridges and } \\
\text { vehicles/equipment as } \\
\text { they pass through. }\end{array}$ & $\begin{array}{l}\text { Firehose connected to } \\
\text { salt water supply can be } \\
\text { connected to a mobile } \\
\text { diffuser mounted on } \\
\text { truck to better direct } \\
\text { wash water. }\end{array}$ \\
\hline & & ADVANTAGES & $\begin{array}{l}\text { Huge capacity. } \\
\text { Pumps up to } \\
20,000 \text { liters per } \\
\text { minute. } \\
\end{array}$ & $\begin{array}{l}\text { Pumps 2,000-50,000 } \\
\text { liters per minute. } \\
\text { Unlimited water } \\
\text { supply, regional use. }\end{array}$ & $\begin{array}{l}\text { Existing supply of } \\
\text { watercraft. }\end{array}$ & $\begin{array}{l}\text { Portable city to } \\
\text { accommodate } \\
\text { workforce. }\end{array}$ & $\begin{array}{l}\text { Large volumes in areas } \\
\text { with beaches. }\end{array}$ & Capacity. & $\begin{array}{l}\text { COTS allows for more } \\
\text { versatile distribution of } \\
\text { water for various water } \\
\text { wash down needs (e.g., } \\
\text { pavement, vehicles). }\end{array}$ \\
\hline & & LIMITATIONS & Availability. & $\begin{array}{l}\text { Water containment. } \\
\text { For immediate coastal } \\
\text { only. Use as } \\
\text { continuous pump for } \\
\text { seawater. }\end{array}$ & $\begin{array}{l}\text { Available certified } \\
\text { operators, available } \\
\text { water pumps, DIY } \\
\text { guidance, } \\
\text { retrofitability, } \\
\text { liability concerns. } \\
\text { Limited to immediate } \\
\text { coastal coverage. }\end{array}$ & $\begin{array}{l}\text { Availability, } \\
\text { decontamination. }\end{array}$ & Region specific. & $\begin{array}{l}\text { Difficult to capture and } \\
\text { contain contaminated } \\
\text { water. }\end{array}$ & None identified. \\
\hline & & R\&D NEEDS & $\begin{array}{l}\text { Survey of inventory } \\
\text { (LR). Compatibility } \\
\text { with salt (LR). }\end{array}$ & $\begin{array}{l}\text { Survey of inventory } \\
\text { (LR). Best practice } \\
\text { guidance (LR) }\end{array}$ & $\begin{array}{l}\text { Liability concerns } \\
\text { lessons from previous } \\
\text { disasters (e.g., Sandy } \\
\text { and Harvey) (LR). } \\
\text { Apps used to recruit } \\
\text { volunteers (LR). } \\
\text { Survey of inventory } \\
\text { (LR). Best practice } \\
\text { guidance (LR). }\end{array}$ & $\begin{array}{l}\text { Survey of inventory } \\
\text { (LR). Decontamination } \\
\text { guidance (LR). }\end{array}$ & $\begin{array}{l}\text { Research ways to } \\
\text { transport large volumes } \\
\text { of sand (LR). }\end{array}$ & $\begin{array}{l}\text { Research ways to contain } \\
\text { contaminated wastewater } \\
\text { on bridge (LR). }\end{array}$ & Guidance on use (PE). \\
\hline
\end{tabular}

All images are courtesy of Shutterstock. 
TABLE 3-2-5 Support Goal: Mitigation of Received Dose to First Responders for Scenario 5

\begin{tabular}{|c|c|c|c|c|c|c|c|c|c|c|c|c|c|c|c|c|}
\hline \multirow{2}{*}{$\begin{array}{c}\text { Scenario } \\
\text { Description }\end{array}$} & \multirow{2}{*}{ Summary } & \multirow{2}{*}{ Category } & \multicolumn{14}{|c|}{ Column Number } \\
\hline & & & 1 & 2 & 3 & 4 & 5 & 6 & 7 & 8 & 9 & 10 & 11 & 12 & 13 & 14 \\
\hline \multirow{5}{*}{$\begin{array}{l}\text { Scenario 5: } \\
\text { Airborne contamination is } \\
\text { a significant source of } \\
\text { radiation dose to } \\
\text { unprotected people. } \\
\text { Because contamination } \\
\text { after settling is primarily } \\
\text { found on horizontal } \\
\text { surfaces (e.g., streets, } \\
\text { walkways, parking lots, } \\
\text { urban green spaces), } \\
\text { reducing the potential for } \\
\text { resuspension is potentially } \\
\text { important. Through prior } \\
\text { research and experience, it } \\
\text { is known that applying a } \\
\text { sprayable polymer coating } \\
\text { (the consistency of wet } \\
\text { paint; e.g., outdoor paints } \\
\text { and epoxies such as those } \\
\text { designed for roadways or } \\
\text { other paved surfaces to } \\
\text { the surfaces effectively } \\
\text { ties down contaminated } \\
\text { particles. How would } \\
\text { polymer coating material } \\
\text { be distributed over an } \\
\text { area of several square } \\
\text { blocks? How would it be } \\
\text { distributed over an area } \\
\text { of several square miles? } \\
\text { How do methods change if } \\
\text { the coating needs to be } \\
\text { distributed over vertical } \\
\text { surfaces such as the } \\
\text { facades of buildings? }\end{array}$} & \multirow{5}{*}{$\begin{array}{l}\text { There are systems } \\
\text { that can be mounted } \\
\text { to trucks to spray } \\
\text { aerosols (e.g., for } \\
\text { mosquito control). } \\
\text { The fire department } \\
\text { has equipment that } \\
\text { can spray foam onto } \\
\text { surfaces, but it is not } \\
\text { known if they can } \\
\text { spray a polymer. } \\
\text { - For much of the } \\
\text { equipment listed, } \\
\text { compatibility with } \\
\text { polymer and the } \\
\text { application of this } \\
\text { coating (coverage } \\
\text { rate and thickness) } \\
\text { are unknowns. } \\
\text { - Sprayer nozzle size } \\
\text { and viscosity of the } \\
\text { coating are } \\
\text { interdependent and } \\
\text { need to be tested to } \\
\text { ensure that the } \\
\text { sprayer nozzzle or } \\
\text { hose will not clog. }\end{array}$} & EQUIPMENT & $\begin{array}{l}\text { Remote sprayer } \\
\text { platform. }\end{array}$ & $\begin{array}{l}\text { Handheld } \\
\text { sprayer. }\end{array}$ & $\begin{array}{l}\text { Medium- } \\
\text { area sprayer. }\end{array}$ & $\begin{array}{l}\text { Large-area } \\
\text { sprayer. }\end{array}$ & $\begin{array}{l}\text { Emulsion } \\
\text { sprayer (liquid } \\
\text { asphalt) } \\
\text { distributors. }\end{array}$ & $\begin{array}{l}\text { Conventiona } \\
1 \text { paint. }\end{array}$ & $\begin{array}{l}\text { Highway } \\
\text { paint. }\end{array}$ & Bambi buckets. & $\begin{array}{l}\text { Pothole patch } \\
\text { trucks. }\end{array}$ & Paver trucks. & $\begin{array}{l}\text { Water tankers } \\
\text { and hoses. }\end{array}$ & $\begin{array}{l}\text { Firefighting } \\
\text { aircraft } \\
\text { dumpers. }\end{array}$ & $\begin{array}{l}\text { Agricultural } \\
\text { sprayers. }\end{array}$ & $\begin{array}{l}\text { IBC } \\
\text { pump/sprayer, } \\
\text { truck } \\
\text { mounted. }\end{array}$ \\
\hline & & DESCRIPTION & 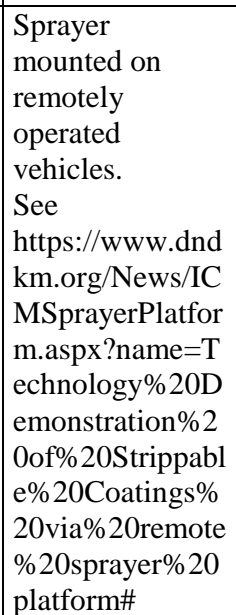 & $\begin{array}{l}\text { Latex and } \\
\text { oil-based } \\
\text { applicators. }\end{array}$ & $\begin{array}{l}\text { Latex and } \\
\text { oil-based } \\
\text { applicators. }\end{array}$ & $\begin{array}{l}\text { Aqueous } \\
\text { sprayer such } \\
\text { as an } \\
\text { industrial } \\
\text { water truck }\end{array}$ & $\begin{array}{l}\text { Sprays asphalt } \\
\text { and with chip } \\
\text { spreader can be } \\
\text { used to seal-in- } \\
\text { place the } \\
\text { contamination. }\end{array}$ & $\begin{array}{l}\text { Provides } \\
\text { rapid cover } \\
\text { of surfaces. }\end{array}$ & $\begin{array}{l}\text { Provides } \\
\text { rapid cover } \\
\text { of surfaces. } \\
\end{array}$ & $\begin{array}{l}\text { Designed to } \\
\text { fight fires by } \\
\text { deluging an } \\
\text { area with water } \\
\text { from a nearby } \\
\text { water source. }\end{array}$ & $\begin{array}{l}\text { Designed for } \\
\text { rapid cover of } \\
\text { small } \\
\text { potholes. }\end{array}$ & $\begin{array}{l}\text { Covers single } \\
\text { lane of } \\
\text { roadway in } \\
\text { one pass with } \\
\text { variable depth } \\
\text { of asphalt. }\end{array}$ & $\begin{array}{l}\text { Provides large } \\
\text { supply of } \\
\text { fluid for } \\
\text { spraying on } \\
\text { surfaces. }\end{array}$ & $\begin{array}{l}\text { Designed to } \\
\text { fight fires by } \\
\text { deluging an } \\
\text { area with } \\
\text { water from a } \\
\text { nearby water } \\
\text { source. }\end{array}$ & $\begin{array}{l}\text { Designed for } \\
\text { distributing } \\
\text { pesticide, } \\
\text { herbicide, or } \\
\text { fertilizer on } \\
\text { fields. } \\
\text { They can } \\
\text { similarly } \\
\text { spread water- } \\
\text { based } \\
\text { solutions. }\end{array}$ & $\begin{array}{l}\text { Versatile } \\
\text { design } \\
\text { mounted on } \\
\text { available } \\
\text { trucks." }\end{array}$ \\
\hline & & ADVANTAGES & $\begin{array}{l}\text { Remote } \\
\text { operation. }\end{array}$ & $\begin{array}{l}\text { Large } \\
\text { quantities } \\
\text { available } \\
\text { for small } \\
\text { applications } \\
\text { (<5-liter } \\
\text { capacity). } \\
\text { Minimal } \\
\text { waste. }\end{array}$ & $\begin{array}{l}\text { Large } \\
\text { quantities } \\
\text { available for } \\
\text { small } \\
\text { applications } \\
\text { ( 20-liter } \\
\text { capacity). } \\
\text { Minimal } \\
\text { waste. }\end{array}$ & $\begin{array}{l}\text { Large-area } \\
\text { applicator } \\
\text { (2,000--iter } \\
\text { capacity). }\end{array}$ & $\begin{array}{l}\text { Sprays liquid } \\
\text { asphalt for } \\
\text { complete } \\
\text { coverage of } \\
\text { roadways. May } \\
\text { spray polymer } \\
\text { coating. }\end{array}$ & $\begin{array}{l}\text { Large } \\
\text { quantities } \\
\text { available } \\
\text { COTS. }\end{array}$ & $\begin{array}{l}\text { Available } \\
\text { COTS. } \\
\text { Designed for } \\
\text { outdoor } \\
\text { paved } \\
\text { surfaces and } \\
\text { resistant to } \\
\text { vehicle } \\
\text { traffic. }\end{array}$ & $\begin{array}{l}\text { Large capacity } \\
\text { (up to } \\
2,600 \text { gallons). }\end{array}$ & $\begin{array}{l}\text { Manual or } \\
\text { automated } \\
\text { trucks, large } \\
\text { quantities } \\
\text { available } \\
\text { COTS. }\end{array}$ & $\begin{array}{l}\text { Large } \\
\text { quantities } \\
\text { available } \\
\text { COTS. }\end{array}$ & $\begin{array}{l}\text { Large } \\
\text { quantities } \\
\text { available } \\
\text { COTS, } \\
\text { variable } \\
\text { capacity } \\
\text { tanks. }\end{array}$ & $\begin{array}{l}\text { Can treat } \\
\text { large areas, } \\
\text { large volumes } \\
\text { (800-20,000 } \\
\text { gallons). }\end{array}$ & $\begin{array}{l}\text { Large } \\
\text { coverage rate. }\end{array}$ & $\begin{array}{l}\text { Versatile for } \\
\text { existing } \\
\text { trucks, large } \\
\text { coverage } \\
\text { rates, on- } \\
\text { vehicle water } \\
\text { supply. }\end{array}$ \\
\hline & & LIMITATIONS & $\begin{array}{l}\text { Compatibility } \\
\text { with polymer, } \\
\text { availability. } \\
\text { Scaled-up } \\
\text { designs not } \\
\text { known. }\end{array}$ & $\begin{array}{l}\text { Small } \\
\text { coverage } \\
\text { rate. }\end{array}$ & $\begin{array}{l}\text { Small } \\
\text { coverage } \\
\text { rate. }\end{array}$ & $\begin{array}{l}\text { Compatibility } \\
\text { with polymer, } \\
\text { availability. }\end{array}$ & $\begin{array}{l}\text { Compatibility } \\
\text { with polymer, } \\
\text { availability. }\end{array}$ & $\begin{array}{l}\text { Ruggedness, } \\
\text { coverage } \\
\text { rate }\end{array}$ & $\begin{array}{l}\text { Coverage } \\
\text { rate and } \\
\text { supply for } \\
\text { gross } \\
\text { coverage of } \\
\text { paved areas. }\end{array}$ & $\begin{array}{l}\text { Gross spreader, } \\
\text { compatibility } \\
\text { with polymer, } \\
\text { availability. } \\
\text { Requires } \\
\text { trained } \\
\text { personnel. The } \\
\text { impact of water } \\
\text { dropped at } \\
\text { elevation can } \\
\text { damage } \\
\text { structures. }\end{array}$ & $\begin{array}{l}\text { Coverage rate, } \\
\text { compatibility } \\
\text { with polymer. }\end{array}$ & $\begin{array}{l}\text { Control over } \\
\text { thickness to } \\
\text { reduce rad } \\
\text { waste upon } \\
\text { removal. }\end{array}$ & $\begin{array}{l}\text { Compatibility } \\
\text { with polymer. }\end{array}$ & $\begin{array}{l}\text { Gross } \\
\text { spreader, } \\
\text { availability, } \\
\text { compatibility } \\
\text { with polymer, } \\
\text { ability to } \\
\text { control layer } \\
\text { thickness. } \\
\text { Requires } \\
\text { trained } \\
\text { personnel. } \\
\text { The impact of } \\
\text { water dropped } \\
\text { at elevation } \\
\text { can damage } \\
\text { structures. }\end{array}$ & $\begin{array}{l}\text { Compatibly } \\
\text { with polymer. }\end{array}$ & $\begin{array}{l}\text { Compatibility } \\
\text { with polymer. }\end{array}$ \\
\hline & & R\&D NEEDS & $\begin{array}{l}\text { Survey of } \\
\text { inventory (LR). } \\
\text { Compatibility } \\
\text { with polymer } \\
\text { (RR, BE, , PE). } \\
\text { Guidance on use } \\
\text { (LR). }\end{array}$ & $\begin{array}{l}\text { Guidance } \\
\text { on use } \\
\text { (LR). }\end{array}$ & $\begin{array}{l}\text { Guidance on } \\
\text { use (LR). }\end{array}$ & $\begin{array}{l}\text { Survey of } \\
\text { inventory } \\
\text { (LR). } \\
\text { Compatibility } \\
\text { with polymer } \\
\text { (LR and/or } \\
\text { BE, PE). } \\
\text { Guidance on } \\
\text { use (LR). }\end{array}$ & $\begin{array}{l}\text { Survey of } \\
\text { inventory (LR). } \\
\text { Compatibility } \\
\text { with polymer } \\
\text { (LR, BE, PE). } \\
\text { Guidance on use } \\
\text { with polymer or } \\
\text { asphalt } \\
\text { emulsions (LR). }\end{array}$ & $\begin{array}{l}\text { Research } \\
\text { effectiveness } \\
\text { (LR, BE, } \\
\text { PE). }\end{array}$ & $\begin{array}{l}\text { Survey of } \\
\text { inventory } \\
\text { (LR). }\end{array}$ & $\begin{array}{l}\text { Survey of } \\
\text { inventory (LR). } \\
\text { Compatibility } \\
\text { with polymer } \\
\text { (LR and/or BE, } \\
\text { PE). Guidance } \\
\text { on use (LR, } \\
\text { BE, PE). }\end{array}$ & $\begin{array}{l}\text { Guidance on } \\
\text { use (LR). } \\
\text { Compatibility } \\
\text { with polymer } \\
\text { (LR and/or } \\
\text { BE, PE). }\end{array}$ & $\begin{array}{l}\text { Compatibility } \\
\text { with polymer } \\
\text { (LR and/or } \\
\text { BE, PE). }\end{array}$ & $\begin{array}{l}\text { Compatibility } \\
\text { with polymer } \\
\text { (LR and/or } \\
\text { BE, PE). }\end{array}$ & $\begin{array}{l}\text { Survey of } \\
\text { inventory } \\
\text { (LR). } \\
\text { Compatibility } \\
\text { with polymer } \\
\text { (LR). } \\
\text { Guidance on } \\
\text { use (LR, BE, } \\
\text { PE). }\end{array}$ & $\begin{array}{l}\text { Survey of } \\
\text { inventory } \\
\text { (LR). } \\
\text { Compatibility } \\
\text { with polymer } \\
\text { (LR, BE, PE). } \\
\text { Guidance on } \\
\text { use (LR, BE, } \\
\text { PE). }\end{array}$ & $\begin{array}{l}\text { Survey of } \\
\text { inventory } \\
\text { (LR). } \\
\text { Compatibility } \\
\text { with polymer } \\
\text { (LR, BE, PE). }\end{array}$ \\
\hline
\end{tabular}


TABLE 3-2-6 Support Goal: Mitigation of Received Dose to First Responders for Scenario 6

\begin{tabular}{|c|c|c|c|c|c|c|c|c|c|}
\hline \multirow{2}{*}{ Scenario Description } & Summary & Category & \multicolumn{7}{|c|}{ Column Number } \\
\hline & \multirow{6}{*}{$\begin{array}{l}\text { - Critical infrastructure } \\
\text { (e.g., power plants, } \\
\text { potable water treatment } \\
\text { plants, sewer treatment } \\
\text { plants, law enforcement } \\
\text { facilities, hospitals) } \\
\text { should be prioritized for } \\
\text { immediate gross } \\
\text { decontamination. They } \\
\text { have heavy foot traffic } \\
\text { and could easily uncover } \\
\text { previously covered } \\
\text { contamination. A short- } \\
\text { or medium-term cleanup } \\
\text { strategy would be } \\
\text { necessary for these } \\
\text { facilities to continue to } \\
\text { operate until the incident } \\
\text { stabilized. } \\
\text { - Hospitals may be } \\
\text { inherently easier to } \\
\text { decontaminate because } \\
\text { of their many hard } \\
\text { surfaces. They also may } \\
\text { already have protocols } \\
\text { in place that could be } \\
\text { implemented. } \\
\text { - Gaps vary according to } \\
\text { type of critical } \\
\text { infrastructure and the } \\
\text { requirements to prevent } \\
\text { damage to sensitive } \\
\text { equipment outside or } \\
\text { within the facility. } \\
\text { - Gaps will likely include } \\
\text { effective ways to collect } \\
\text { and contain wastewater } \\
\text { and movement of } \\
\text { airborne contamination. } \\
\text { For drinking water, } \\
\text { wastewater and } \\
\text { stormwater collection } \\
\text { systems, see McAfee } \\
\text { and Biwer. }\end{array}$} & & 1 & $\frac{2}{d \text { train } r}-x-r$ & 3 & 4 & 5 & 6 & 7 \\
\hline \multirow{5}{*}{$\begin{array}{l}\text { Scenario 6: } \\
\text { How would the } \\
\text { equipment and } \\
\text { methods in Scenarios } \\
1-3 \text { (gravel, mulch, } \\
\text { sand, fresh water, salt } \\
\text { water) and } 5 \\
\text { (sprayable polymer } \\
\text { coating) differ if the } \\
\text { contamination } \\
\text { occurred at critical } \\
\text { infrastructure such } \\
\text { as a hospital, water } \\
\text { reclamation facility, } \\
\text { drinking water plant, } \\
\text { airport, or } \\
\text { communications } \\
\text { center? }\end{array}$} & & EQUIPMENT & Mobile wash units. & $\begin{array}{l}\text { tunnel wash cars. } \\
\text { tors }\end{array}$ & Jet engine blowers. & Vacuum trains. & Easier-to-clean surfaces. & Bridges. & Airport sprayer systems. \\
\hline & & DESCRIPTION & $\begin{array}{l}\text { Supplies wash waters } \\
\text { dispensed by manned- } \\
\text { hose over small to } \\
\text { medium areas. }\end{array}$ & $\begin{array}{l}\text { Spray jets clean the rail } \\
\text { lines and scrub or wash } \\
\text { tunnels. }\end{array}$ & $\begin{array}{l}\text { Can remove debris from } \\
\text { tracks with powerful } \\
\text { airflow. }^{\text {a }}\end{array}$ & $\begin{array}{l}\text { Trains designed to remove } \\
\text { debris from rail lines. }\end{array}$ & $\begin{array}{l}\text { Hospitals and other } \\
\text { infrastructure with smooth, } \\
\text { hard surfaces are more } \\
\text { easily decontaminated. }\end{array}$ & $\begin{array}{l}\text { Can use existing standpipes } \\
\text { or build ad hoc systems to } \\
\text { spray fixatives or } \\
\text { decontaminants onto vehicles } \\
\text { (e.g., cars, trucks, rail stock, } \\
\text { planes) and equipment (e.g., } \\
\text { emergency response } \\
\text { equipment, cargo } \\
\text { containers).c }\end{array}$ & $\begin{array}{l}\text { Deicing equipment and } \\
\text { aircraft washing capabilities } \\
\text { can decontaminate surfaces. } \\
\text { Military airbases often have } \\
\text { built-in wash ramps on the } \\
\text { runway. }\end{array}$ \\
\hline & & ADVANTAGES & Mobile. & $\begin{array}{l}\text { Specialized to infra- } \\
\text { structure. }\end{array}$ & $\begin{array}{l}\text { Powerful, large coverage } \\
\text { rates, specialized to } \\
\text { infrastructure. }\end{array}$ & $\begin{array}{l}\text { Large coverage rate, } \\
\text { specialized to } \\
\text { infrastructure. }\end{array}$ & $\begin{array}{l}\text { Current techniques for } \\
\text { cleaning and washing may } \\
\text { be sufficient. }\end{array}$ & $\begin{array}{l}\text { Large coverage rate, } \\
\text { specialized to infrastructure. }\end{array}$ & $\begin{array}{l}\text { Specialized to infrastructure. } \\
\text { Current techniques for } \\
\text { cleaning and washing may } \\
\text { be sufficient. }\end{array}$ \\
\hline & & LIMITATIONS & Local prevalence. & $\begin{array}{l}\text { Water collection, } \\
\text { effectiveness. }\end{array}$ & $\begin{array}{l}\text { Limited numbers, } \\
\text { uncontrolled movement } \\
\text { of material. }\end{array}$ & $\begin{array}{l}\text { Local prevalence, } \\
\text { filtration efficiency. }\end{array}$ & $\begin{array}{l}\text { Guidance specific to } \\
\text { critical infra-structure. }\end{array}$ & $\begin{array}{l}\text { Water collection, guidance } \\
\text { specific to critical infra- } \\
\text { structure. }\end{array}$ & $\begin{array}{l}\text { Water collection, guidance } \\
\text { specific to critical } \\
\text { infrastructure. }\end{array}$ \\
\hline & & R\&D NEEDS & $\begin{array}{l}\text { Survey of inventory } \\
\text { (LR). }\end{array}$ & $\begin{array}{l}\text { Water collection/ } \\
\text { containment methods } \\
\text { (LR). Dose reduction } \\
\text { assessment (LR, PE). }\end{array}$ & $\begin{array}{l}\text { Survey of inventory } \\
\text { (LR). Collection of } \\
\text { material (LR, PE). }\end{array}$ & $\begin{array}{l}\text { Filtration efficacy (LR). } \\
\text { Survey of inventory (LR). }\end{array}$ & $\begin{array}{l}\text { Best practice guidance } \\
\text { from power plants, } \\
\text { hospitals, etc. (LR). }\end{array}$ & Best practice guidance. & Best practice guidance. \\
\hline
\end{tabular}

All images are courtesy of Shutterstock, except: (a) Metro Transit Authority New York Creative Commons and permission granted from (b) NEU, Inc., and (c) New York Department of Transportation.

McFee, J., Krishnan, R., Piao, H., \& Randall, P. (2008). Decontamination Methods for Drinking Water Treatment and Distribution Systems. Wiley Handbook of Science and Technology for Homeland Security, 1-24.

Biwer, B. M., Chen, S. Y., Monette, F. A., MacKinney, J., \& Janke, R. (2008). Decontamination Methods for Wastewater and Stormwater Collection and Treatment Systems. Wiley Handbook of Science and Technology for Homeland Security, 1-26. 
TABLE 3-2-7 Support Goal: Mitigation of Received Dose to First Responders for Scenario 7

\begin{tabular}{|c|c|c|c|c|c|c|c|c|}
\hline \multirow{2}{*}{$\begin{array}{c}\text { Scenario } \\
\text { Description }\end{array}$} & \multirow{2}{*}{ Summary } & \multirow{2}{*}{ Category } & \multicolumn{6}{|c|}{ Column Number } \\
\hline & & & 1 & 2 & 3 & 4 & 5 & 6 \\
\hline \multirow{5}{*}{$\begin{array}{l}\text { Scenario 7: } \\
\text { Airborne contamination is a } \\
\text { significant source of } \\
\text { radiation dose to } \\
\text { unprotected people. Because } \\
\text { contamination after settling } \\
\text { is primarily found on } \\
\text { horizontal surfaces (e.g., } \\
\text { streets, walkways, parking } \\
\text { lots, urban green spaces), } \\
\text { reducing the potential for } \\
\text { resuspension is potentially } \\
\text { important. Areas of concern } \\
\text { would be covered in } \\
\text { contaminated debris and } \\
\text { loose particles. In some } \\
\text { areas, the debris would be } \\
\text { substantial and could be } \\
\text { removed by bulldozer-type } \\
\text { equipment. In other areas, } \\
\text { the debris material could be } \\
\text { swept and vacuumed. How } \\
\text { can contaminated debris } \\
\text { be physically moved or } \\
\text { removed? }\end{array}$} & \multirow{5}{*}{$\begin{array}{l}\text { - In small areas, portable containment } \\
\text { tents could be erected for dry } \\
\text { cleanup work using HEPA } \\
\text { vacuums, shovels, etc., and air } \\
\text { handling systems with HEPA filters } \\
\text { may be constructed. } \\
\text { - Large inflatable tents could isolate } \\
\text { and contain dust over large areas. } \\
\text { Additional hazards must be } \\
\text { assessed (e.g., wind). Portable } \\
\text { containment tents may not be an } \\
\text { option where heavy lifting } \\
\text { equipment or tools are in use. } \\
\text { - Airborne hazards could be } \\
\text { controlled using misting } \\
\text { technology, ranging from cheap } \\
\text { misting hoses (for gardens) to } \\
\text { industrial misting systems set up } \\
\text { over larger areas. Misting } \\
\text { suppresses airborne contamination, } \\
\text { but wastewater containment would } \\
\text { be necessary because contamination } \\
\text { is more mobile when dissolved or } \\
\text { suspended in liquid. } \\
\text { - Street sweepers use both wet and } \\
\text { dry technologies and would be } \\
\text { suitable for use in damp } \\
\text { environments. } \\
\text { - Common concerns with the items } \\
\text { used to remove debris (e.g., a street } \\
\text { sweeper or HEPA vacuum) include } \\
\text { removal efficiency based on } \\
\text { particle characteristics, dust control, } \\
\text { and worker protection. }\end{array}$} & EQUIPMENT & $\begin{array}{l}\text { Portable } \\
\text { decontamination } \\
\text { tent. }\end{array}$ & Large tent. & $\begin{array}{l}\text { Water spray or } \\
\text { misting equipment } \\
\text { (see Scenario } 2 \\
\text { equipment). }\end{array}$ & $\begin{array}{l}\text { Household-type } \\
\text { HEPA } \\
\text { vacuums. }\end{array}$ & Road sweepers. & $\begin{array}{l}\text { Bulldozers, } \\
\text { backhoes, front } \\
\text { loaders, bobcats, } \\
\text { etc. }\end{array}$ \\
\hline & & DESCRIPTION & $\begin{array}{l}\begin{array}{l}\text { Could contain } \\
\text { small hotspots. }\end{array} \\
\end{array}$ & $\begin{array}{l}\text { Could isolate and } \\
\text { contain dust over } \\
\text { larger plots. }\end{array}$ & $\begin{array}{l}\text { Reduces dust } \\
\text { generation from } \\
\text { vehicle movement. }\end{array}$ & $\begin{array}{l}\text { Smaller area } \\
\text { removal of dust } \\
\text { and small debris } \\
\text { plus HEPA } \\
\text { filters. }\end{array}$ & $\begin{array}{l}\text { Provides mist of } \\
\text { water to control dust. }\end{array}$ & $\begin{array}{l}\text { Moves debris into } \\
\text { piles or into } \\
\text { hauling vehicles. }\end{array}$ \\
\hline & & ADVANTAGES & $\begin{array}{l}\text { Custom and ad hoc } \\
\text { systems available. }\end{array}$ & $\begin{array}{l}\text { Large area } \\
\text { containment. }\end{array}$ & $\begin{array}{l}\text { See Scenario } 2 \\
\text { equipment. }\end{array}$ & $\begin{array}{l}\text { Nationwide } \\
\text { distributors. } \\
\text { Recent efficacy } \\
\text { data is } \\
\text { available. }\end{array}$ & $\begin{array}{l}\text { Large quantities } \\
\text { available COTS, } \\
\text { large coverage rates. } \\
\text { Suppresses dust. } \\
\text { Collects water. }\end{array}$ & $\begin{array}{l}\text { Large coverage } \\
\text { rates, large } \\
\text { quantities available } \\
\text { for use. }\end{array}$ \\
\hline & & LIMITATIONS & Coverage rate. & Availability. & $\begin{array}{l}\text { See Scenario } 2 \\
\text { equipment. May not } \\
\text { provide enough } \\
\text { volume or force to } \\
\text { physically move } \\
\text { debris. }\end{array}$ & $\begin{array}{l}\text { Effectiveness a } \\
\text { function of } \\
\text { particle } \\
\text { characteristics, } \\
\text { small coverage } \\
\text { rate. }\end{array}$ & $\begin{array}{l}\text { May be ineffective on } \\
\text { small particles. } \\
\text { Exhaust could spread } \\
\text { contamination. }\end{array}$ & $\begin{array}{l}\text { Hermetically } \\
\text { sealed cabs not } \\
\text { COTS. }\end{array}$ \\
\hline & & R\&D NEEDS & None identified. & $\begin{array}{l}\text { Survey of } \\
\text { inventory (LR). }\end{array}$ & $\begin{array}{l}\text { Survey of inventory } \\
\text { (LR). Practicality } \\
\text { (LR). Guidance on } \\
\text { use (BE, PE) }\end{array}$ & $\begin{array}{l}\text { Research } \\
\text { removal } \\
\text { efficiency (LR). }\end{array}$ & $\begin{array}{l}\text { Research removal } \\
\text { efficiency (LR and } \\
\text { BE, PE). Guidance on } \\
\text { use (LR, BE, PE). } \\
\text { Methods to control } \\
\text { dust and emissions } \\
\text { (LR). } \\
\text { Decontamination } \\
\text { guidance (LR). }\end{array}$ & $\begin{array}{l}\text { PPE needs to be } \\
\text { addressed (LR). } \\
\text { Cab design or } \\
\text { retrofit for } \\
\text { contaminated } \\
\text { environments (LR } \\
\text { and/or BE, PE). } \\
\text { Dose to driver on } \\
\text { routes (LR). } \\
\text { Decontamination } \\
\text { guidance (LR). }\end{array}$ \\
\hline
\end{tabular}

All images are courtesy of Shutterstock. 
TABLE 3-2-8 Support Goal: Mitigation of Received Dose to First Responders for Scenario 8

\begin{tabular}{|c|c|c|c|c|c|}
\hline \multirow{2}{*}{$\begin{array}{c}\text { Scenario } \\
\text { Description }\end{array}$} & \multirow[b]{2}{*}{ Summary } & \multirow[b]{2}{*}{ Category } & \multicolumn{3}{|c|}{ Column Number } \\
\hline & & & 1 & 2 & 3 \\
\hline \multirow{5}{*}{$\begin{array}{l}\text { Scenario 8: } \\
\text { Regardless of scenario, how } \\
\text { would the above equipment and } \\
\text { methods differ if the } \\
\text { contamination occurred along } \\
\text { egress routes that are necessary } \\
\text { to transport people away from the } \\
\text { affected area (for example, major } \\
\text { highways)? }\end{array}$} & \multirow{5}{*}{$\begin{array}{l}\text { - On key egress routes, gross decontamination } \\
\text { would be deployed rather than coverings for } \\
\text { dose mitigation or refined decontamination } \\
\text { techniques. } \\
\text { - Pressure washing would be a key way to } \\
\text { remove as much primary contamination as } \\
\text { possible and prevent resuspension of dry } \\
\text { particulates from passing vehicles. } \\
\text { - After pressure washing, paint or other } \\
\text { durable surface coatings could fix residual } \\
\text { contamination in place until egress or } \\
\text { evacuation was complete and access more } \\
\text { controlled. The whole surface could then be } \\
\text { lifted for disposal in necessary. } \\
\text { - Common issues include capturing and } \\
\text { containing large amounts of contaminated } \\
\text { water and finding effective coatings to cover } \\
\text { contamination. }\end{array}$} & EQUIPMENT & Water wash-down activities. & Post-cleaning stabilization. & Fireboats and bridges. \\
\hline & & DESCRIPTION & $\begin{array}{l}\text { Spraying water will only move } \\
\text { activity to drainage near the roads. }\end{array}$ & $\begin{array}{l}\text { Following pressure washing, paint or } \\
\text { other durable surface coatings can } \\
\text { stabilize contamination. }\end{array}$ & $\begin{array}{l}\text { Use of fireboats can decontaminate } \\
\text { bridges and passing cars. }\end{array}$ \\
\hline & & ADVANTAGES & Availability. & Availability. & Capacity. \\
\hline & & LIMITATIONS & $\begin{array}{l}\text { It is difficult to capture and contain } \\
\text { large amounts of contaminated } \\
\text { water. }\end{array}$ & $\begin{array}{l}\text { Coating effective for radiation. Waste } \\
\text { may be generated if coated material is } \\
\text { disposed later. }\end{array}$ & $\begin{array}{l}\text { It is difficult to capture and contain } \\
\text { contaminated water. }\end{array}$ \\
\hline & & R\&D NEEDS & $\begin{array}{l}\text { Water collection and containment } \\
\text { methods (LR). Dose reduction } \\
\text { estimates (LR, PE). }\end{array}$ & Coating effectiveness (LR, BE, PE). & $\begin{array}{l}\text { Contain contaminated wastewater on } \\
\text { bridge }(\mathrm{LR}) \text {. }\end{array}$ \\
\hline
\end{tabular}

All images are courtesy of Shutterstock.

TABLE 3-2-9 Support Goal: Mitigation of Received Dose to First Responders for Scenario 9

\begin{tabular}{|c|c|c|c|c|c|c|c|c|}
\hline Scenario 9 & \multicolumn{8}{|c|}{ Responses } \\
\hline \begin{tabular}{|l|} 
Question to participants: \\
Do you have other \\
thoughts or specific \\
questions based on your \\
experiences in your \\
geographical area related to \\
this topic?
\end{tabular} & $\begin{array}{l}\text { Washing down surfaces } \\
\text { or covering surfaces, } \\
\text { with whatever agent, } \\
\text { generates very } \\
\text { significant amounts of } \\
\text { secondary waste in the } \\
\text { form of contaminated } \\
\text { liquids and solids so tie- } \\
\text { down coatings and paints } \\
\text { are best option. }\end{array}$ & $\begin{array}{l}\text { The use of salt addition } \\
\text { requires very careful } \\
\text { consideration. Surface } \\
\text { water usually goes into } \\
\text { rivers and streams after } \\
\text { passing through water } \\
\text { treatment works, but } \\
\text { without any salt removal. } \\
\text { Hence, there is a } \\
\text { significant environmental } \\
\text { detriment. }\end{array}$ & $\begin{array}{l}\text { Sprayer nozzle size and } \\
\text { viscosity of coating are } \\
\text { interdependent and hence } \\
\text { a high risk exists that } \\
\text { improvised sprayers may } \\
\text { become clogged. } \\
\text { Therefore, any wide-area } \\
\text { spraying devices need to } \\
\text { be identified in advance } \\
\text { for the materials to be } \\
\text { used. }\end{array}$ & $\begin{array}{l}\text { Not all polymer coatings } \\
\text { are equal, and some may } \\
\text { fragment rather than } \\
\text { adhere strongly after } \\
\text { application. }\end{array}$ & $\begin{array}{l}\text { May need to consider that } \\
\text { remediation personnel may be } \\
\text { present in same area as public. } \\
\text { Such staff are likely to be wearing } \\
\text { PPE and possibly respiratory } \\
\text { protective equipment that may not } \\
\text { have been provided to the public } \\
\text { in the same location. } \\
\text { This may cause confusion and } \\
\text { concern. Issuing everyone a dust } \\
\text { mask and plastic gloves, as } \\
\text { obtained from all DIY stores, will } \\
\text { provide basic protection. }\end{array}$ & $\begin{array}{l}\text { Any areas where waste } \\
\text { accumulates may need to } \\
\text { be shielded in advance or } \\
\text { selected to minimize } \\
\text { rediation to the general } \\
\text { environment. }\end{array}$ & $\begin{array}{l}\text { Under as low as reasonably } \\
\text { achievable (ALARA) } \\
\text { principles, early consideration } \\
\text { needs to be given to waste } \\
\text { transport or final disposal } \\
\text { containers to reduce the dose } \\
\text { associated with multiple } \\
\text { repacking operations. }\end{array}$ & $\begin{array}{l}\text { Rain frequency. The potential } \\
\text { effects of rain frequency on } \\
\text { mitigation will need to be } \\
\text { understood and considered. }\end{array}$ \\
\hline
\end{tabular}


TABLE 3-2-10 Support Goal Training: Mitigation of Received Dose to First Responders for Scenarios 1-9

\begin{tabular}{|c|c|c|c|c|c|c|}
\hline Support Goal Training: Scenarios 1-9 & Summary & \multicolumn{5}{|c|}{ Responses } \\
\hline $\begin{array}{l}\text { Question to participants: } \\
\text { What are your thoughts and } \\
\text { recommendations on availability of } \\
\text { trained human assets and training } \\
\text { of additional assets that will likely be } \\
\text { needed in order to accomplish the } \\
\text { scenarios under this Goal? (It is } \\
\text { understood that training will be a } \\
\text { significant effort and an additional } \\
\text { limiting factor in any response } \\
\text { scenario. Further, it may need to be } \\
\text { addressed more thoroughly in the } \\
\text { future, but input is needed to help } \\
\text { guide how training guidance should be } \\
\text { developed.) }\end{array}$ & $\begin{array}{l}\text { Wide-area radiological } \\
\text { contamination incidents } \\
\text { are rare, and a response to } \\
\text { such an incident will } \\
\text { require tremendous } \\
\text { human assets. Lessons } \\
\text { learned from the cleanup } \\
\text { efforts in Japan show that } \\
\text { many thousands of } \\
\text { individuals each day are } \\
\text { engaged in clean-up } \\
\text { activities. }\end{array}$ & $\begin{array}{l}\text { Contamination control and } \\
\text { radiation safety training would } \\
\text { need to be given to all } \\
\text { personnel working within the } \\
\text { remediation area to ensure that } \\
\text { operations progress efficiently } \\
\text { and dose to individuals is } \\
\text { minimized. This will need to be } \\
\text { regularly refreshed, and } \\
\text { individuals will need to be } \\
\text { supervised until they gain } \\
\text { sufficient experience. }\end{array}$ & $\begin{array}{l}\text { A common training program and } \\
\text { procedure could be developed for } \\
\text { rollout to the public and agencies } \\
\text { to ensure a consistent approach by } \\
\text { all parties and to reduce risk of } \\
\text { confusion. }\end{array}$ & $\begin{array}{l}\text { Local areas where remediation } \\
\text { operations are underway are controlled } \\
\text { to prevent public access as this would } \\
\text { otherwise lead to increased risks and } \\
\text { reduce the effectiveness of remediation } \\
\text { work (e.g., due to cross-contamination } \\
\text { risks). }\end{array}$ & $\begin{array}{l}\text { Limited companies offer NVQ Levels } 2 / 3 \text { for } \\
\text { Radiation Protection and Nuclear } \\
\text { Decommissioning and a Level } 2 \text { for Process } \\
\text { Industry Operations. Sections of a typical } \\
\text { NVQ syllabus could be extracted to meet the } \\
\text { requirements of different roles. }\end{array}$ & $\begin{array}{l}\text { The general approach to grade the level } \\
\text { of training required could be considered, } \\
\text { as per the Survey \& Monitoring Goal. If } \\
\text { the contamination is truly at this real } \\
\text { scale, then the first major resource is } \\
\text { likely to come from the military, } \\
\text { supported by fire and rescue services and } \\
\text { civilian experts. Basic training in } \\
\text { radiological protection can then be given } \\
\text { to pertinent personnel. Their training } \\
\text { will focus on operator safety. }\end{array}$ \\
\hline
\end{tabular}




\subsection{SUPPORT GOAL: DECONTAMINATION (GROSS AND FINAL)}

Decontamination methods can be more effective if implemented within days of a release rather than waiting months or years. Waiting can allow the contamination to evolve chemically and physically, rendering it more difficult to remove. What types of municipal and commercial equipment can carry out gross or final decontamination of contaminated surfaces? Examples include asphalt-milling machines to remove the top layer of road surfaces, bobcats to remove the top layer of vegetation or soil, and tillers to turn over contaminated soil. Note that "gross decontamination" is a type of decontamination conducted with the goal of reducing contamination levels. This reduction may not be sufficient for final cleanup levels, but it may be useful to mitigate some public hazard or to contain contamination. Tables 3-3-1 to 3-3-7 present various scenarios related to decontamination, possible responses to the scenarios, and possible equipment/technology used in responding, including its advantages, limitations, and R\&D needs. 
TABLE 3-3-1 Support Goal: Decontamination (gross and final) for Scenario

\begin{tabular}{|c|c|c|c|c|c|c|c|c|c|c|c|c|c|c|}
\hline \multirow{2}{*}{$\begin{array}{c}\text { Scenario } \\
\text { Description }\end{array}$} & \multirow{2}{*}{ Summary } & \multirow{2}{*}{ Category } & \multicolumn{12}{|c|}{ Column Number } \\
\hline & & & 1 & 2 & 3 & 4 & 5 & 6 & 7 & 8 & 9 & 10 & 11 & 12 \\
\hline \multirow{5}{*}{$\begin{array}{l}\text { Scenario 1: } \\
\text { EPA studies } \\
\text { identified that soil } \\
\text { is a major source } \\
\text { of contaminated } \\
\text { material in } \\
\text { decontamination } \\
\text { efforts in an urban } \\
\text { environment. Prior } \\
\text { research and } \\
\text { experience suggest } \\
\text { that nearly all } \\
\text { contamination can } \\
\text { be removed by } \\
\text { removing less than } \\
5 \text { cm (2 inches) of } \\
\text { soil. This } \\
\text { equipment would } \\
\text { be useful in } \\
\text { removing a very } \\
\text { shallow layer of } \\
\text { soil, grass, or } \\
\text { foliage from large } \\
\text { urban green } \\
\text { spaces such as } \\
\text { playgrounds and } \\
\text { open grass fields. }\end{array}$} & \multirow{5}{*}{ 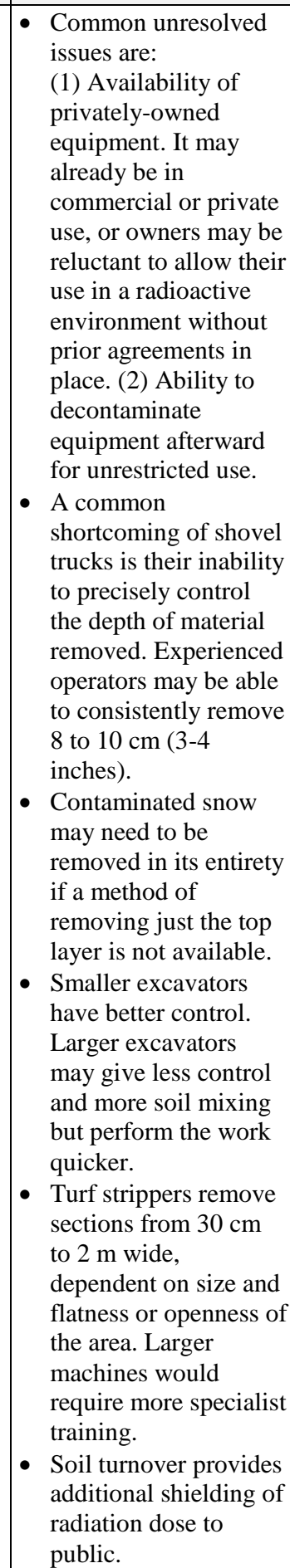 } & EQUIPMENT & Bobcats. & $\begin{array}{l}\text { Small } \\
\text { mechanical } \\
\text { digares }\end{array}$ & $\begin{array}{l}\text { Large } \\
\text { excavators. }\end{array}$ & Turf stripper. & $\begin{array}{l}\text { Vacuum } \\
\text { trucks. }\end{array}$ & Bulldozer. & $\begin{array}{l}\text { Conveyor } \\
\text { segmented gate } \\
\text { systems }\end{array}$ & $\begin{array}{l}\text { Crop duster or } \\
\text { mobile spray for } \\
\text { defoliation }\end{array}$ & $\begin{array}{l}\text { Soil stabilizer } \\
\text { sprays. }\end{array}$ & $\begin{array}{l}\text { Soil turnover, } \\
\text { medium scale. }\end{array}$ & $\begin{array}{l}\text { Soil turnover, larger } \\
\text { scale. }\end{array}$ & Deep ploughing. \\
\hline & & DESCRIPTION & $\begin{array}{l}\begin{array}{l}\text { Small } \\
\text { earthmover to } \\
\text { clear debris or } \\
\text { move } \\
\text { contaminated } \\
\text { solid waste. }\end{array} \\
\end{array}$ & $\begin{array}{l}\text { Small } \\
\text { earthmover to } \\
\text { clear debris, } \\
\text { move } \\
\text { contaminated } \\
\text { solid waste, or } \\
\text { dig a trench or } \\
\text { hole. }\end{array}$ & $\begin{array}{l}\text { Large } \\
\text { earthmover to } \\
\text { clear debris, } \\
\text { move } \\
\text { contaminated } \\
\text { solid waste, } \\
\text { or dig a } \\
\text { trench or } \\
\text { hole. }\end{array}$ & $\begin{array}{l}\text { Removes a } \\
\text { layer of grass } \\
\text { for disposal. }\end{array}$ & $\begin{array}{l}\text { Can remove } \\
\text { loose soil, } \\
\text { gravel, dust, } \\
\text { leaves, or } \\
\text { small debris } \\
\text { into on-board } \\
\text { storage tank. }\end{array}$ & $\begin{array}{l}\text { Common } \\
\text { construction } \\
\text { equipment for } \\
\text { pushing earth. } \\
\text { Can be used to } \\
\text { scrape top layer } \\
\text { of earth for } \\
\text { removal. }\end{array}$ & $\begin{array}{l}\text { Used to reduce the } \\
\text { amount of solid } \\
\text { waste by assaying } \\
100 \% \text { of soil or } \\
\text { debris. }\end{array}$ & $\begin{array}{l}\text { Can defoliate a } \\
\text { wide or medium } \\
\text { area of } \\
\text { contaminated } \\
\text { leaves for } \\
\text { collection. }\end{array}$ & $\begin{array}{l}\text { Hardens top layer of } \\
\text { soil to reduce dust } \\
\text { and/or facilitate its } \\
\text { removal as a } \\
\text { hardened layer. }\end{array}$ & $\begin{array}{l}\text { Small plows } \\
\text { attached to small or } \\
\text { large vehicles can } \\
\text { turn over the soil } \\
\text { down to a depth of } \\
\text { approximately } 15 \\
\text { cm ( } 6 \text { inches) to } \\
\text { bury radioactivity } \\
\text { and shield against } \\
\text { radiation. }\end{array}$ & $\begin{array}{l}\text { Tractor-towed } \\
\text { plows ( } 6 \text { to } 10 \\
\text { furrows) can turn } \\
\text { over the soil up to } \\
25 \text { to } 30 \mathrm{~cm} \text { (10 to } \\
12 \text { inches) deep to } \\
\text { bury radioactivity } \\
\text { and shield against } \\
\text { radiation. }\end{array}$ & $\begin{array}{l}\text { Largest plows } \\
\text { pulled by } \\
\text { specialty tractors } \\
\text { can reach } 50 \mathrm{~cm} \\
(21 \text { inches }) \text { in } \\
\text { depth or more to } \\
\text { bury radioactivity } \\
\text { and shield against } \\
\text { radiation. }\end{array}$ \\
\hline & & ADVANTAGES & $\begin{array}{l}\text { Fast, efficient } \\
\text { with a range } \\
\text { of bucket } \\
\text { sizes. }\end{array}$ & $\begin{array}{l}\text { Combination } \\
\text { excavator and } \\
\text { grading plane } \\
\text { useful for } \\
\text { breaking up } \\
\text { and removing } \\
\text { layers. }\end{array}$ & $\begin{array}{l}\text { Greater than } \\
\text { 1-ton bucket, } \\
\text { heavy lifting. }\end{array}$ & $\begin{array}{l}\text { Large area turf } \\
\text { stripper with } \\
\text { controlled } \\
\text { depth. } \\
\text { Removing grass } \\
\text { in early } \\
\text { response can be } \\
\text { very effective at } \\
\text { removing } \\
\text { contamination. }\end{array}$ & $\begin{array}{l}\text { Better control } \\
\text { than shovel } \\
\text { trucks for } \\
\text { removing } \\
\text { loose debris. }\end{array}$ & $\begin{array}{l}\text { Larger coverage } \\
\text { with less-precise } \\
\text { control than } \\
\text { smaller shovel } \\
\text { trucks. }\end{array}$ & $\begin{array}{l}\text { Assay 100\% of } \\
\text { material, } \\
\text { radionuclide } \\
\text { discrimination, high } \\
\text { throughput. } \\
\text { Specifically } \\
\text { developed and } \\
\text { proven in } \\
\text { radioactive soil } \\
\text { applications. }\end{array}$ & $\begin{array}{l}\text { Used in some } \\
\text { cities. Large } \\
\text { coverage rates. }\end{array}$ & $\begin{array}{l}\text { COTS, large } \\
\text { coverage rate, } \\
\text { rugged. }\end{array}$ & $\begin{array}{l}\text { Large quantities } \\
\text { available COTS, } \\
\text { good control for } \\
\text { medium plots, DIY. }\end{array}$ & $\begin{array}{l}\text { Large coverage rate, } \\
\text { large quantities } \\
\text { available COTS in } \\
\text { rural areas. }\end{array}$ & $\begin{array}{l}\text { Deep turnover } \\
\text { provides highest } \\
\text { dose reduction. } \\
\text { Prior efficacy } \\
\text { data available. }\end{array}$ \\
\hline & & LIMITATIONS & Depth control. & Depth control. & $\begin{array}{l}\text { Depth } \\
\text { control. }\end{array}$ & Removal rate. & $\begin{array}{l}\text { Limited } \\
\text { capacity (less } \\
\text { than } \\
5000 \text { gallons), } \\
\text { availability in } \\
\text { numbers. } \\
\end{array}$ & Depth control. & $\begin{array}{l}\text { Cost-benefit, } \\
\text { availability. }\end{array}$ & $\begin{array}{l}\text { Availability, public } \\
\text { acceptance. } \\
\text { Requires trained } \\
\text { personnel. }\end{array}$ & $\begin{array}{l}\text { Little data on } \\
\text { effectiveness. }\end{array}$ & $\begin{array}{l}\text { Dust control, does } \\
\text { not remove } \\
\text { contamination, } \\
\text { sufficient depth to } \\
\text { reduce exposure. }\end{array}$ & $\begin{array}{l}\text { Availability, dust } \\
\text { control, sufficient } \\
\text { depth to reduce } \\
\text { exposure. Does not } \\
\text { remove } \\
\text { contamination. }\end{array}$ & $\begin{array}{l}\text { Availability, dust } \\
\text { control. Does not } \\
\text { remove } \\
\text { contamination. }\end{array}$ \\
\hline & & $R \& D$ NEEDS & $\begin{array}{l}\text { Best practice } \\
\text { guidance } \\
\text { (LR). }\end{array}$ & $\begin{array}{l}\text { Best practice } \\
\text { guidance } \\
\text { (LR). }\end{array}$ & $\begin{array}{l}\text { Best practice } \\
\text { guidance } \\
\text { (LR). }\end{array}$ & $\begin{array}{l}\text { Best practice } \\
\text { guidance (LR). }\end{array}$ & $\begin{array}{l}\text { Best practice } \\
\text { guidance } \\
\text { (LR). Survey } \\
\text { of inventory } \\
\text { (LR). Clarify } \\
\text { transport } \\
\text { regulations } \\
\text { (LR). }\end{array}$ & $\begin{array}{l}\text { Best practice } \\
\text { guidance (LR). }\end{array}$ & $\begin{array}{l}\text { Cost benefit analysis } \\
\text { compared to other } \\
\text { options (LR). } \\
\text { Availability (LR). }\end{array}$ & $\begin{array}{l}\text { Survey of } \\
\text { inventory (LR). }\end{array}$ & $\begin{array}{l}\text { Data from } \\
\text { Fukushima } \\
\text { experience (LR). } \\
\text { Efficacy for } \\
\text { radionuclide } \\
\text { removal } \\
\text { (formulation, depth, } \\
\text { and spray } \\
\text { parameters) (LR, } \\
\text { BE). }\end{array}$ & $\begin{array}{l}\text { Exposure reduction } \\
\text { estimates (LR). } \\
\text { Expected uptake of } \\
\text { radionuclides into } \\
\text { grasses, plants, and } \\
\text { trees (LR). Best } \\
\text { practice guidance } \\
\text { (LR). }\end{array}$ & $\begin{array}{l}\text { Exposure reduction } \\
\text { estimates (LR). } \\
\text { Expected uptake of } \\
\text { radionuclides into } \\
\text { grasses, plants, and } \\
\text { trees (LR). Survey } \\
\text { of inventory (LR). } \\
\text { Best practice } \\
\text { guidance (LR). }\end{array}$ & $\begin{array}{l}\text { Survey of } \\
\text { inventory (LR). } \\
\text { Best practice } \\
\text { guidance (LR). }\end{array}$ \\
\hline
\end{tabular}


TABLE 3-3-1 (Cont.)

\begin{tabular}{|c|c|c|c|c|c|c|c|c|c|c|}
\hline \multirow{2}{*}{$\begin{array}{c}\text { Scenario } \\
\text { Description } \\
\end{array}$} & \multirow{2}{*}{ Summary } & \multirow{2}{*}{ Category } & \multicolumn{8}{|c|}{ Column Number } \\
\hline & & & 13 & 14 & 15 & 16 & 17 & 18 & 19 & 20 \\
\hline & & EQUIPMENT & $\begin{array}{l}\text { Beach cleaning } \\
\text { machines. }\end{array}$ & Snow plows. & $\begin{array}{l}\text { Current city } \\
\text { resources for fallen } \\
\text { tree removal. }\end{array}$ & Booms. & $\begin{array}{l}\text { Residential } \\
\text { mowers. }\end{array}$ & Tractor mower. & Hedge cutters. & $\begin{array}{l}\text { Lawn sweeper/ } \\
\text { vacuum. }\end{array}$ \\
\hline & & DESCRIPTION & $\begin{array}{l}\text { Equipment } \\
\text { removes the top } \\
\text { layer of sand and } \\
\text { sifts it to remove } \\
\text { large objects. } \\
\text { May be adapted } \\
\text { to remove sand } \\
\text { layer. }\end{array}$ & $\begin{array}{l}\text { Can remove } \\
\text { contaminated snow } \\
\text { or be repurposed to } \\
\text { move soil and } \\
\text { vegetation. }\end{array}$ & $\begin{array}{l}\text { Available assets to } \\
\text { lift, trim, cut, } \\
\text { remove, mulch, and } \\
\text { haul foliage and } \\
\text { trees. }\end{array}$ & $\begin{array}{l}\text { Elevated lift to } \\
\text { facilitate } \\
\text { collection, } \\
\text { washing of } \\
\text { trees and } \\
\text { buildings, etc. }\end{array}$ & $\begin{array}{l}\text { Lawn mowers of } \\
\text { various size can } \\
\text { cut and remove } \\
\text { contaminated } \\
\text { grass. }\end{array}$ & $\begin{array}{l}\text { Large mowers } \\
\text { can cut and } \\
\text { remove } \\
\text { contaminated } \\
\text { grass. }\end{array}$ & $\begin{array}{l}\text { Hand tools like } \\
\text { hedge cutters and } \\
\text { shears can remove } \\
\text { contaminated } \\
\text { foliage. }\end{array}$ & $\begin{array}{l}\text { Designed to } \\
\text { remove leaves and } \\
\text { mulch from short } \\
\text { grass. }\end{array}$ \\
\hline & & ADVANTAGES & $\begin{array}{l}\text { Large coverage } \\
\text { rate, specialty } \\
\text { equipment. } \\
\text { Efficacy data } \\
\text { available. }\end{array}$ & $\begin{array}{l}\text { Large quantities } \\
\text { available COTS in } \\
\text { colder climates, } \\
\text { large coverage rate. }\end{array}$ & $\begin{array}{l}\text { City resources, } \\
\text { specialized and } \\
\text { trained staff, variety } \\
\text { of tasks. }\end{array}$ & $\begin{array}{l}\text { Large } \\
\text { quantities } \\
\text { available } \\
\text { COTS. }\end{array}$ & $\begin{array}{l}\text { Large quantities } \\
\text { available, DIY, } \\
\text { collects } \\
\text { clippings. }\end{array}$ & $\begin{array}{l}\text { Existing } \\
\text { network, regular } \\
\text { operating } \\
\text { schedule, trained } \\
\text { operators, large } \\
\text { coverage rate. }\end{array}$ & $\begin{array}{l}\text { Large quantities } \\
\text { available, DIY. }\end{array}$ & $\begin{array}{l}\text { DIY, good } \\
\text { coverage rate. } \\
\text { Designed to handle } \\
\text { leaves, twigs, and } \\
\text { other small plant } \\
\text { debris. }\end{array}$ \\
\hline & & LIMITATIONS & $\begin{array}{l}\text { Availability, dust } \\
\text { control. Requires } \\
\text { modification } \\
\text { (binder) to } \\
\text { remove } \\
\text { contaminated top } \\
\text { layer. }\end{array}$ & $\begin{array}{l}\text { Availability in } \\
\text { warmer climates, } \\
\text { depth control. }\end{array}$ & Dust control. & $\begin{array}{l}\text { None } \\
\text { identified. }\end{array}$ & $\begin{array}{l}\text { Dust control, } \\
\text { operator } \\
\text { exposure, only } \\
\text { removes } \\
\text { contamination on } \\
\text { grass blades. } \\
\end{array}$ & $\begin{array}{l}\text { Drops clippings. } \\
\text { Dust control, } \\
\text { operator } \\
\text { exposure, only } \\
\text { removes } \\
\text { contamination on } \\
\text { grass blades. } \\
\end{array}$ & Collecting clippings. & $\begin{array}{l}\text { May not be } \\
\text { effective on grass } \\
\text { clippings. }\end{array}$ \\
\hline & & R\&D NEEDS & $\begin{array}{l}\text { Binder materials } \\
\text { to create } \\
\text { separable surface } \\
\text { layer (LRS, BE). } \\
\text { Survey of } \\
\text { inventory (LR). }\end{array}$ & $\begin{array}{l}\text { Disposal plan } \\
\text { options and } \\
\text { guidance (LR). }\end{array}$ & $\begin{array}{l}\text { Best practice } \\
\text { guidance (LR). }\end{array}$ & $\begin{array}{l}\text { None } \\
\text { identified. }\end{array}$ & $\begin{array}{l}\text { Best practice } \\
\text { guidance (LR). }\end{array}$ & $\begin{array}{l}\text { Best practice } \\
\text { guidance (LR). }\end{array}$ & $\begin{array}{l}\text { Best practice } \\
\text { guidance (LR). }\end{array}$ & $\begin{array}{l}\text { Best practice } \\
\text { guidance (LR). }\end{array}$ \\
\hline
\end{tabular}

All images are courtesy of Shutterstock, except: (a) permission granted from ANTECH. 
TABLE 3-3-2 Support Goal: Decontamination (gross and final) for Scenario 2

\begin{tabular}{|c|c|c|c|c|c|c|c|c|c|c|c|c|c|c|c|c|}
\hline \multirow{2}{*}{$\begin{array}{c}\text { Scenario } \\
\text { Description } \\
\end{array}$} & \multirow{2}{*}{ Summary } & \multirow[b]{2}{*}{ Category } & \multicolumn{14}{|c|}{ Column Number } \\
\hline & & & 1 & 2 & 3 & 4 & 5 & 6 & 7 & 8 & 9 & 10 & 11 & 12 & 13 & 14 \\
\hline \multirow{5}{*}{\begin{tabular}{|l} 
Scenario 2: \\
Same as \\
Scenario 1, \\
except that the \\
intent is to \\
identify \\
equipment that \\
would be \\
useful in \\
removing a \\
very shallow \\
layer of soil, \\
grass, or \\
foliage from \\
smaller, \\
discontinuous \\
plots o orban \\
green spaces \\
such as front \\
and back yards \\
and garden \\
areas.
\end{tabular}} & \multirow{5}{*}{$\begin{array}{l}\text { - Common unresolved } \\
\text { issues are: (1) } \\
\text { Availability of } \\
\text { privately owned } \\
\text { equipment. It may } \\
\text { already be in } \\
\text { commercial or private } \\
\text { use, or owners may be } \\
\text { reluctant to allow use in } \\
\text { a radioactive } \\
\text { environment without } \\
\text { prior agreements in } \\
\text { place. (2) Ability to } \\
\text { decontaminate } \\
\text { equipment afterward } \\
\text { for unrestricted use. } \\
\text { - Where specialist } \\
\text { remediation support is } \\
\text { not required, DIY } \\
\text { options may be } \\
\text { important. Shovels, } \\
\text { hoses, spray washers, } \\
\text { and wipes supplied to } \\
\text { landowners are cost- } \\
\text { effective methods of } \\
\text { remediation. For DIY } \\
\text { equipment, a best } \\
\text { practices guidance is } \\
\text { not available and would } \\
\text { need to be published to } \\
\text { reduce risk. } \\
\text { - Power washers can } \\
\text { remove contamination } \\
\text { from hard surfaces like } \\
\text { rock gardens, rock } \\
\text { lawns, pavers, } \\
\text { driveways, siding, and } \\
\text { roofs. Guidance is } \\
\text { needed regarding } \\
\text { manner of use (llow } \\
\text { rates, pressure, } \\
\text { coverage rate, water } \\
\text { collection and storage). }\end{array}$} & EQUIPMENT & Shovel. & Bobcats. & $\begin{array}{l}\text { Small } \\
\text { mechanical } \\
\text { diggers. }\end{array}$ & Turf stripper. & $\begin{array}{l}\text { Soil turnover, } \\
\text { small scale. }\end{array}$ & $\begin{array}{l}\text { Soil turnover, } \\
\text { medium scale. }\end{array}$ & Vacuum trucks. & Hand wiping. & $\begin{array}{l}\text { Pressure } \\
\text { washing. }\end{array}$ & $\begin{array}{l}\text { Brushing/ } \\
\text { scrubbing. }\end{array}$ & $\begin{array}{l}\text { Conveyor } \\
\text { segmented } \\
\text { gate systems. }\end{array}$ & $\begin{array}{l}\text { Residential } \\
\text { mowers. }\end{array}$ & $\begin{array}{l}\text { Hedge } \\
\text { cutters. }\end{array}$ & $\begin{array}{l}\text { Lawn } \\
\text { sweeper/ } \\
\text { vacuum. }\end{array}$ \\
\hline & & DESCRIPTION & $\begin{array}{l}\text { Manual } \\
\text { removal of } \\
\text { contamin- } \\
\text { ated solids. }\end{array}$ & $\begin{array}{l}\text { Small } \\
\text { earthmover to } \\
\text { clear debris or } \\
\text { move } \\
\text { contaminated } \\
\text { solid waste. }\end{array}$ & $\begin{array}{l}\text { Small } \\
\text { earthmover to } \\
\text { clear debris or } \\
\text { move } \\
\text { contaminated } \\
\text { solid waste, or } \\
\text { dig a trench or } \\
\text { hole. }\end{array}$ & $\begin{array}{l}\text { Removes a } \\
\text { layer of grass } \\
\text { for disposal. }\end{array}$ & $\begin{array}{l}\text { Hand tools like a } \\
\text { shovel or fork can } \\
\text { turn over soil in } \\
\text { small plots to bury } \\
\text { radioactivity and } \\
\text { shield against } \\
\text { radiation. }\end{array}$ & $\begin{array}{l}\text { Small plows } \\
\text { attached to small or } \\
\text { large vehicles can } \\
\text { turn over the soil } \\
\text { down to a depth of } \\
\text { approximately } \\
6 \text { inches to bury } \\
\text { radioactivity and } \\
\text { shield against } \\
\text { radiation. }\end{array}$ & $\begin{array}{l}\text { Can remove } \\
\text { loose } \\
\text { contaminated } \\
\text { soil, gravel, } \\
\text { dust, leaves, or } \\
\text { small debris into } \\
\text { on-board storage } \\
\text { tank }\end{array}$ & $\begin{array}{l}\text { Contaminated } \\
\text { dust can be } \\
\text { removed by } \\
\text { wiping with } \\
\text { wet towels. }\end{array}$ & $\begin{array}{l}\text { Removes } \\
\text { contaminated } \\
\text { dust on or } \\
\text { shallow layer } \\
\text { of surfaces of } \\
\text { rock gardens, } \\
\text { trees/foliage, } \\
\text { etc. }\end{array}$ & $\begin{array}{l}\text { Removes } \\
\text { contaminated } \\
\text { dust on } \\
\text { surfaces of } \\
\text { rock gardens, } \\
\text { patios, roofs, } \\
\text { gutters, } \\
\text { windows, } \\
\text { walls, and } \\
\text { other hard } \\
\text { materials. }\end{array}$ & $\begin{array}{l}\text { Used to } \\
\text { reduce the } \\
\text { amount of } \\
\text { low-level } \\
\text { waste by } \\
\text { assaying } \\
100 \% \text { of soil } \\
\text { or debris." }\end{array}$ & $\begin{array}{l}\text { Lawn mowers } \\
\text { of various size } \\
\text { can cut and } \\
\text { remove } \\
\text { contaminated } \\
\text { grass. }\end{array}$ & $\begin{array}{l}\text { Hand tools } \\
\text { like hedge } \\
\text { cutters and } \\
\text { shears can } \\
\text { remove } \\
\text { contam- } \\
\text { inated } \\
\text { foliage. }\end{array}$ & $\begin{array}{l}\text { Designed to } \\
\text { remove leaves } \\
\text { and mulch } \\
\text { from short } \\
\text { grass. }\end{array}$ \\
\hline & & ADVANTAGES & $\begin{array}{l}\text { Cheap. } \\
\text { Requires } \\
\text { minimal } \\
\text { training. }\end{array}$ & $\begin{array}{l}\text { Fast, efficient } \\
\text { with a range } \\
\text { of bucket } \\
\text { sizes, very } \\
\text { effective. }\end{array}$ & $\begin{array}{l}\text { Combination } \\
\text { excavator and } \\
\text { grading plane } \\
\text { useful for } \\
\text { breaking up } \\
\text { and removing } \\
\text { layers. }\end{array}$ & $\begin{array}{l}\text { Large area } \\
\text { turf stripper } \\
\text { with } \\
\text { controlled } \\
\text { depth. } \\
\text { Removing } \\
\text { grass in early } \\
\text { response can } \\
\text { be very } \\
\text { effective at } \\
\text { removing } \\
\text { contamination }\end{array}$ & $\begin{array}{l}\text { Large quantitites } \\
\text { available COTS, } \\
\text { good control for } \\
\text { small plots, DIY. }\end{array}$ & $\begin{array}{l}\text { Large quantities } \\
\text { available COTS, } \\
\text { good control for } \\
\text { medium plots, DIY. }\end{array}$ & $\begin{array}{l}\text { Better control } \\
\text { than shovel } \\
\text { trucks to remove } \\
\text { loose debris. }\end{array}$ & $\begin{array}{l}\text { Large } \\
\text { quantities } \\
\text { available } \\
\text { COTS, } \\
\text { effective, } \\
\text { minimizes } \\
\text { waste, DIY. }\end{array}$ & $\begin{array}{l}\text { Large } \\
\text { quantities } \\
\text { available } \\
\text { COTS, } \\
\text { minimizes } \\
\text { water use, } \\
\text { DIY, } \\
\text { Fukushima } \\
\text { experience. }\end{array}$ & $\begin{array}{l}\text { Large } \\
\text { quantities } \\
\text { available } \\
\text { COTS, } \\
\text { minimizes } \\
\text { water use, } \\
\text { DIY. }\end{array}$ & $\begin{array}{l}\text { Assay } 100 \% \\
\text { of material, } \\
\text { radionuclide } \\
\text { discrim., } \\
\text { high } \\
\text { throughput. }\end{array}$ & $\begin{array}{l}\text { Large } \\
\text { quantities } \\
\text { available, DIY, } \\
\text { collects } \\
\text { clippings. }\end{array}$ & $\begin{array}{l}\text { Large } \\
\text { quantitites } \\
\text { available } \\
\text { DIY. }\end{array}$ & $\begin{array}{l}\text { DIY, good } \\
\text { coverage rate. } \\
\text { Designed to } \\
\text { handle leaves, } \\
\text { twigs, and } \\
\text { other small } \\
\text { plant debris. }\end{array}$ \\
\hline & & LIMITATIONS & \begin{tabular}{|l} 
Low \\
removal \\
rate.
\end{tabular} & Depth control. & Depth control. & Removal rate. & $\begin{array}{l}\text { Dust control, } \\
\text { sufficient depth to } \\
\text { reduce exposure, } \\
\text { best practice } \\
\text { procedures. }\end{array}$ & $\begin{array}{l}\text { Dust control, } \\
\text { sufficient depth to } \\
\text { reduce exposure. }\end{array}$ & $\begin{array}{l}\text { Limited capacity } \\
\text { (less than } \\
5000 \text { gallons), } \\
\text { availability in } \\
\text { numbers. }\end{array}$ & $\begin{array}{l}\text { Exposure, } \\
\text { manpower, } \\
\text { consistency of } \\
\text { results, best } \\
\text { practice } \\
\text { procedures. }\end{array}$ & $\begin{array}{l}\text { Water } \\
\text { collection, } \\
\text { manpower, } \\
\text { aerosol } \\
\text { exposure, best } \\
\text { practice } \\
\text { procedure. }\end{array}$ & $\begin{array}{l}\text { Water } \\
\text { collection, } \\
\text { manpower, } \\
\text { aerosol } \\
\text { exposure, best } \\
\text { practice } \\
\text { procedure. }\end{array}$ & $\begin{array}{l}\text { Cost-benefit, } \\
\text { availability. }\end{array}$ & $\begin{array}{l}\text { Dust control, } \\
\text { operator } \\
\text { exposure, only } \\
\text { removes } \\
\text { contamination } \\
\text { on grass } \\
\text { blades. }\end{array}$ & $\begin{array}{l}\text { Collecting } \\
\text { clippings. }\end{array}$ & $\begin{array}{l}\text { May not be } \\
\text { effective on } \\
\text { grass } \\
\text { clippings. }\end{array}$ \\
\hline & & R\&D NEEDS & $\begin{array}{l}\text { Best } \\
\text { practice } \\
\text { guidance } \\
\text { (LR). }\end{array}$ & $\begin{array}{l}\text { Best practice } \\
\text { guidance } \\
\text { (LR). }\end{array}$ & $\begin{array}{l}\text { Best practice } \\
\text { guidance } \\
\text { (LR). }\end{array}$ & $\begin{array}{l}\text { Best practice } \\
\text { guidance } \\
\text { (LR). }\end{array}$ & $\begin{array}{l}\text { Exposure reduction } \\
\text { estimates (LR). } \\
\text { Expected uptake of } \\
\text { radionuclides into } \\
\text { grasses, plants, and } \\
\text { trees (LR). Best } \\
\text { practice guidance } \\
\text { (LR). }\end{array}$ & $\begin{array}{l}\text { Exposure reduction } \\
\text { estimates (LR). } \\
\text { Expected uptake of } \\
\text { radionuclides into } \\
\text { grasses, plants, and } \\
\text { trees (LR). Best } \\
\text { practice guidance } \\
\text { (LR). }\end{array}$ & $\begin{array}{l}\text { Survey of } \\
\text { inventory (LR). } \\
\text { Clarify transport } \\
\text { regulations } \\
\text { (LR). } \\
\text { Decontaminatio } \\
\text { n guidance (LR). } \\
\text { Best practice } \\
\text { guidance (LR). }\end{array}$ & $\begin{array}{l}\text { Data from } \\
\text { Fukushima } \\
\text { experience } \\
\text { (LR). Best } \\
\text { practice } \\
\text { guidance } \\
\text { (LR). }\end{array}$ & $\begin{array}{l}\text { Data from } \\
\text { Fukushima } \\
\text { experience } \\
\text { (LR). Best } \\
\text { practice } \\
\text { guidance } \\
\text { (LR). }\end{array}$ & $\begin{array}{l}\text { Data from } \\
\text { Fukushima } \\
\text { experience } \\
\text { (LR). Best } \\
\text { practice } \\
\text { guidance } \\
\text { (LR). }\end{array}$ & $\begin{array}{l}\text { Cost-benefit } \\
\text { analysis } \\
\text { (LR). Survey } \\
\text { of inventory } \\
\text { (LR). }\end{array}$ & $\begin{array}{l}\text { Best practice } \\
\text { guidance (LR). }\end{array}$ & $\begin{array}{l}\text { Best } \\
\text { practice } \\
\text { guidance } \\
\text { (LR). }\end{array}$ & $\begin{array}{l}\text { Best practice } \\
\text { guidance } \\
\text { (LR). }\end{array}$ \\
\hline
\end{tabular}


TABLE 3-3-3 Support Goal: Decontamination (gross and final) for Scenario 3

\begin{tabular}{|c|c|c|c|c|c|c|c|c|c|c|c|c|c|c|c|c|c|c|c|}
\hline \multirow{2}{*}{$\begin{array}{c}\text { Scenario } \\
\text { Description } \\
\end{array}$} & \multirow{2}{*}{ Summary } & \multirow{2}{*}{ ategory } & \multicolumn{17}{|c|}{ Column Number } \\
\hline & & & 1 & 2 & 3 & 4 & 5 & 6 & 7 & 8 & 8 & 10 & 11 & 12 & 13 & 14 & 15 & 16 & 17 \\
\hline \multirow{5}{*}{$\begin{array}{l}\text { Scenario 3: } \\
\text { Hard, } \\
\text { horizontal } \\
\text { surfaces such } \\
\text { as roads, } \\
\text { walkways, and } \\
\text { parking lots } \\
\text { can trap } \\
\text { radioactive } \\
\text { contamination. } \\
\text { Prior studies } \\
\text { and experience } \\
\text { show that the } \\
\text { contamination } \\
\text { resides at or } \\
\text { very near the } \\
\text { surface of such } \\
\text { hard materials } \\
\text { (less than } 1 \mathrm{~cm} \\
\text { or less than } \\
0 \text {.5 inches). } \\
\text { What } \\
\text { equipment } \\
\text { would be } \\
\text { useful for } \\
\text { removing } \\
\text { contamination } \\
\text { at the surface? } \\
\text { What } \\
\text { equipment } \\
\text { would be } \\
\text { useful in } \\
\text { removing a } \\
\text { very shallow } \\
\text { depth of } \\
\text { paving } \\
\text { material? How } \\
\text { does the choice } \\
\text { of equipment } \\
\text { and method } \\
\text { change if many } \\
\text { linear miles of } \\
\text { surfaces need } \\
\text { such treatment? }\end{array}$} & \multirow{5}{*}{$\begin{array}{l}\text { Common } \\
\text { unresolved } \\
\text { issues: } \\
\text { (1) Availability } \\
\text { of privately- } \\
\text { owned } \\
\text { equipment. It } \\
\text { may already be } \\
\text { in commercial } \\
\text { or private use, } \\
\text { or owners may } \\
\text { be reluctant to } \\
\text { allowits use in } \\
\text { a radioactive } \\
\text { environment } \\
\text { without prior } \\
\text { agreements in } \\
\text { place. } \\
\text { (2) Ability to } \\
\text { decontaminate } \\
\text { equipment } \\
\text { afterward for } \\
\text { unrestricted } \\
\text { use. } \\
\text { Hard surface } \\
\text { removal is very } \\
\text { effective, but } \\
\text { common } \\
\text { unresolved } \\
\text { issues are: } \\
\text { (1) collecting } \\
\text { surface debris, } \\
\text { (2) controlling } \\
\text { dust/ } \\
\text { overspray, } \\
\text { (3) controlling } \\
\text { the depth of } \\
\text { removal to less } \\
\text { than } 1 \mathrm{~cm} \text { or } \\
\text { less, and } \\
\text { (4) small } \\
\text { amount of } \\
\text { equipment } \\
\text { available. }\end{array}$} & EQUIPMENT & $\begin{array}{l}\text { Asphalt } \\
\text { Milling } \\
\text { Machine. }\end{array}$ & $\begin{array}{l}\text { Floor } \\
\text { scabbler. }\end{array}$ & Scarifier. & $\begin{array}{l}\text { Hand-held } \\
\text { planers, } \\
\text { scabblers, } \\
\text { scraper. }\end{array}$ & $\begin{array}{l}\text { Dust } \\
\text { control. }\end{array}$ & $\begin{array}{l}\text { Vehicle } \\
\text { mounted } \\
\text { planer. }\end{array}$ & cavators. & 1lldozer. & $\begin{array}{l}\text { ssure } \\
\text { shing. }\end{array}$ & $\begin{array}{l}\text { ater jetting } \\
\text { hicles. }\end{array}$ & $\begin{array}{l}\text { Shot } \\
\text { blasting. }\end{array}$ & $\begin{array}{l}\text { Emulsion } \\
\text { sprayer } \\
\text { (liquid } \\
\text { asphalt) } \\
\text { distributors. }\end{array}$ & $\begin{array}{l}\text { tge chip } \\
\text { eader. }\end{array}$ & $\begin{array}{l}\text { Chemicals } \\
\text { to remove } \\
\text { concrete } \\
\text { coatings. }\end{array}$ & $\begin{array}{l}\text { Remove } \\
\text { paving } \\
\text { slabs. }\end{array}$ & $\begin{array}{l}\text { Other } \\
\text { specialty } \\
\text { equipment } \\
\text { for concrete } \\
\text { removal. }\end{array}$ & $\begin{array}{l}\text { Washing, } \\
\text { wiping by } \\
\text { hand or } \\
\text { machine. }\end{array}$ \\
\hline & & DESCRIPTION & \begin{tabular}{|l} 
Asphalt \\
milling \\
machines are \\
typically \\
used for \\
removing \\
tarmac \\
surfaces or \\
pavement. \\
\end{tabular} & $\begin{array}{l}\text { Multiple- } \\
\text { tipped heads } \\
\text { pound } \\
\text { surface for } \\
\text { very shallow } \\
\text { removal. }\end{array}$ & $\begin{array}{l}\text { Cutting } \\
\text { wheel } \\
\text { removes } \\
\text { shallow } \\
\text { surface. Also } \\
\text { known as } \\
\text { planers, } \\
\text { milling } \\
\text { machines, or } \\
\text { rotary } \\
\text { cutters. }\end{array}$ & $\begin{array}{l}\text { Hand tools } \\
\text { used for } \\
\text { small- to } \\
\text { medium-sized } \\
\text { areas or areas } \\
\text { that are } \\
\text { harder to } \\
\text { access. }\end{array}$ & $\begin{array}{l}\text { Necessary } \\
\text { to capture } \\
\text { dust } \\
\text { generated } \\
\text { from } \\
\text { surface } \\
\text { removal } \\
\text { machines. }\end{array}$ & $\begin{array}{l}\text { Could strip } \\
\text { the surface } \\
\text { of long } \\
\text { linear areas. }\end{array}$ & $\begin{array}{l}\text { Common } \\
\text { construction } \\
\text { equipment } \\
\text { for digging } \\
\text { and } \\
\text { handling. }\end{array}$ & $\begin{array}{l}\text { Common } \\
\text { construction } \\
\text { equipment } \\
\text { that can push } \\
\text { broken } \\
\text { pavement. }\end{array}$ & $\begin{array}{l}\text { At highest } \\
\text { pressures, can } \\
\text { ablate hard } \\
\text { porous } \\
\text { surfaces (e.g., } \\
\text { asphalt, } \\
\text { concrete, } \\
\text { ceramics, } \\
\text { natural stone) }\end{array}$ & $\begin{array}{l}\text { Cuts the top } \\
\text { layers of } \\
\text { hard surfaces } \\
\text { for removal. }\end{array}$ & $\begin{array}{l}\text { Iron shot or } \\
\text { dry ice } \\
\text { scarifies } \\
\text { paved } \\
\text { surface } \\
\text { layer.. }\end{array}$ & $\begin{array}{l}\text { Sprays } \\
\text { asphalt and } \\
\text { with chip } \\
\text { spreader can } \\
\text { be used to } \\
\text { seal-in-place } \\
\text { the } \\
\text { contamin- } \\
\text { ation. }\end{array}$ & $\begin{array}{l}\text { Spreads } \\
\text { stone chips. } \\
\text { Used with } \\
\text { sealer } \\
\text { distributor to } \\
\text { repave road } \\
\text { and seal-in- } \\
\text { place } \\
\text { contamin- } \\
\text { ation. }\end{array}$ & $\begin{array}{l}\text { Use of } \\
\text { chemicals } \\
\text { to remove } \\
\text { coatings } \\
\text { and top } \\
\text { layers } \\
\text { from } \\
\text { concrete. }\end{array}$ & $\begin{array}{l}\text { If } \\
\text { contamin- } \\
\text { ation of } \\
\text { pavers is } \\
\text { high, may } \\
\text { want to } \\
\text { remove } \\
\text { pavers } \\
\text { without } \\
\text { decontam- } \\
\text { ination. }\end{array}$ & 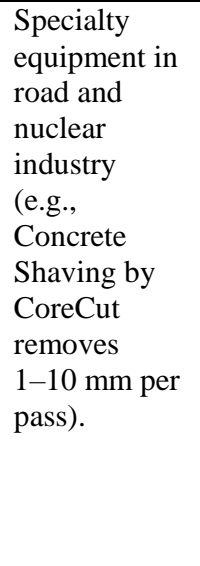 & $\begin{array}{l}\text { DIY methods } \\
\text { using towels, } \\
\text { mops, scrub } \\
\text { brushes. } \\
\text { Mechanical } \\
\text { floor cleaners } \\
\text { may be used } \\
\text { on interior } \\
\text { surfaces to } \\
\text { remove } \\
\text { contaminated } \\
\text { dust. }\end{array}$ \\
\hline & & ADVANTAGES & $\begin{array}{l}\text { Variable } \\
>50 \mathrm{~mm}, \\
\text { small to } \\
\text { medium } 500 \\
\text { to } 1200 \mathrm{~mm} .\end{array}$ & $\begin{array}{l}\text { 5-mm } \\
\text { surface } \\
\text { removal, 20- } \\
30 \text { square } \\
\text { meters per } \\
\text { hour. }\end{array}$ & $\begin{array}{l}5 \text {-mm } \\
\text { surface } \\
\text { removal, 30- } \\
150 \text { square } \\
\text { meters per } \\
\text { hour. }\end{array}$ & $\begin{array}{l}\text { 5-mm surface } \\
\text { removal, } \\
\text { small area } \\
\text { hotspot } \\
\text { removal. } \\
\text { Removes } \\
\text { contaminated } \\
\text { paint. }\end{array}$ & $\begin{array}{l}\text { 60-liter } \\
\text { capacity, } \\
\text { HEPA } \\
\text { filtration } \\
\text { and liquid } \\
\text { collecting } \\
\text { capacity. }\end{array}$ & $\begin{array}{l}\text { Larger } \\
\text { coverage } \\
\text { rate than } \\
\text { hand units, } \\
\text { shallow } \\
\text { removal } \\
\text { depth. }\end{array}$ & $\begin{array}{l}\text { Variable } \\
\text { capacity, } \\
\text { Large } \\
\text { quantities } \\
\text { available }\end{array}$ & $\begin{array}{l}\text { Larger } \\
\text { coverage } \\
\text { with less } \\
\text { precise } \\
\text { control than } \\
\text { smaller } \\
\text { shovel } \\
\text { trucks, very } \\
\text { effective. }\end{array}$ & $\begin{array}{l}\text { Large } \\
\text { quantities } \\
\text { available } \\
\text { COTS, DIY, } \\
\text { Fukushima } \\
\text { experience. } \\
\text { Minimizes } \\
\text { water use. }\end{array}$ & $\begin{array}{l}\text { Very } \\
\text { effective, } \\
\text { Fukushima } \\
\text { experience, } \\
\text { water } \\
\text { collection on } \\
\text { some units. } \\
\text { Removes } \\
\text { surface with } \\
\text { depth } \\
\text { control. } \\
\end{array}$ & $\begin{array}{l}\text { Very } \\
\text { effective, } \\
\text { good } \\
\text { coverage } \\
\text { rate, } \\
\text { Fukushima } \\
\text { experience. } \\
\text { Often has } \\
\text { dust control } \\
\text { unit, }\end{array}$ & $\begin{array}{l}\text { Large rate of } \\
\text { coverage. } \\
\text { Fully } \\
\text { automated. }\end{array}$ & $\begin{array}{l}\text { Large rate of } \\
\text { coverage. } \\
\text { Fully } \\
\text { automated } \\
\text { with } \\
\text { conveyors to } \\
\text { spread } \\
\text { aggregate. }\end{array}$ & $\begin{array}{l}\text { COTS. } \\
\text { Designed } \\
\text { to remove } \\
\text { tough } \\
\text { coatings. }\end{array}$ & $\begin{array}{l}\text { Effective, } \\
\text { DIY. }\end{array}$ & $\begin{array}{l}\text { Specialized } \\
\text { equipment, } \\
\text { very } \\
\text { effective. }\end{array}$ & $\begin{array}{l}\text { Can be } \\
\text { effective on } \\
\text { hard,smooth } \\
\text { surfaces, large } \\
\text { quantities } \\
\text { available } \\
\text { COTS, DIY, } \\
\text { variety of } \\
\text { techniques. }\end{array}$ \\
\hline & & LIMITATIONS & Availability. & $\begin{array}{l}\text { Coverage } \\
\text { rate, dust } \\
\text { control, } \\
\text { availability } \\
\text { in the } \\
\text { numbers } \\
\text { needed. } \\
\text { Defaces } \\
\text { structures. }\end{array}$ & $\begin{array}{l}\text { Dust control, } \\
\text { availability } \\
\text { in the } \\
\text { numbers } \\
\text { needed. }\end{array}$ & $\begin{array}{l}\text { Dust control, } \\
\text { coverage rate, } \\
\text { availability in } \\
\text { the numbers } \\
\text { needed for } \\
\text { roadway } \\
\text { surfaces, DIY } \\
\text { guidance. }\end{array}$ & $\begin{array}{l}\text { Availability } \\
\text { at HEPA } \\
\text { rating. }\end{array}$ & $\begin{array}{l}\text { Dust } \\
\text { control, } \\
\text { availability. }\end{array}$ & $\begin{array}{l}\text { Depth } \\
\text { control. }\end{array}$ & $\begin{array}{l}\text { Depth } \\
\text { control. }\end{array}$ & $\begin{array}{l}\text { Water } \\
\text { collection, } \\
\text { manpower, } \\
\text { aerosol } \\
\text { exposure, best } \\
\text { practice } \\
\text { procedure. }\end{array}$ & $\begin{array}{l}\text { Very } \\
\text { speceialized, } \\
\text { availability, } \\
\text { water } \\
\text { collection. }\end{array}$ & $\begin{array}{l}\text { Very } \\
\text { specialized, } \\
\text { availability, } \\
\text { dust control. }\end{array}$ & $\begin{array}{l}\text { Secondary } \\
\text { waste from } \\
\text { removal of } \\
\text { layer, } \\
\text { availability. }\end{array}$ & Availability. & $\begin{array}{l}\text { Limited to } \\
\text { removal } \\
\text { of } \\
\text { coatings. }\end{array}$ & $\begin{array}{l}\text { Guidance } \\
\text { for use. } \\
\text { May also } \\
\text { need to } \\
\text { remove } \\
\text { grout or } \\
\text { sand under } \\
\text { or between } \\
\text { pavers. }\end{array}$ & $\begin{array}{l}\text { Availability, } \\
\text { types and } \\
\text { variety needs } \\
\text { inventory. }\end{array}$ & $\begin{array}{l}\text { No guidance } \\
\text { available, best } \\
\text { practices } \\
\text { undefined. }\end{array}$ \\
\hline & & R\&D NEEDS & $\begin{array}{l}\text { Survey of } \\
\text { inventory } \\
\text { (LR). }\end{array}$ & $\begin{array}{l}\text { Survey of } \\
\text { inventory } \\
\text { (LR). } \\
\text { Compatible } \\
\text { dust control } \\
\text { options } \\
\text { (LR). }\end{array}$ & $\begin{array}{l}\text { Survey of } \\
\text { inventory } \\
\text { (LR). } \\
\text { Compatible } \\
\text { dust control } \\
\text { options } \\
\text { (LR). }\end{array}$ & $\begin{array}{l}\text { Compatible } \\
\text { dust control } \\
\text { options (LR). } \\
\text { Best practice } \\
\text { guidance } \\
\text { (LR). Survey } \\
\text { of inventory } \\
\text { (LR). }\end{array}$ & $\begin{array}{l}\text { Best } \\
\text { practice } \\
\text { guidance } \\
\text { (LR). } \\
\text { Survey of } \\
\text { inventory } \\
\text { (LR). }\end{array}$ & $\begin{array}{l}\text { Survey of } \\
\text { inventory } \\
\text { (LR). } \\
\text { Compatible } \\
\text { dust control } \\
\text { options } \\
\text { (LR). }\end{array}$ & $\begin{array}{l}\text { Best practice } \\
\text { guidance } \\
\text { (LR). }\end{array}$ & $\begin{array}{l}\text { Best practice } \\
\text { guidance } \\
\text { (LR). }\end{array}$ & $\begin{array}{l}\text { Data from } \\
\text { Fukushima } \\
\text { experience } \\
\text { (LR). Best } \\
\text { practice } \\
\text { guidance } \\
\text { (LR). }\end{array}$ & $\begin{array}{l}\text { Survey of } \\
\text { inventory } \\
\text { (LR). } \\
\text { Compatible } \\
\text { dust control } \\
\text { options } \\
\text { (LR). Best } \\
\text { practice } \\
\text { guidance } \\
\text { (LR). }\end{array}$ & $\begin{array}{l}\text { Survey of } \\
\text { inventory } \\
\text { (LR). } \\
\text { Compatible } \\
\text { dust control } \\
\text { options } \\
\text { (LR). Best } \\
\text { practice } \\
\text { guidance } \\
\text { (LR). }\end{array}$ & $\begin{array}{l}\text { Survey of } \\
\text { inventory } \\
\text { (LR). Best } \\
\text { practice } \\
\text { guidance } \\
\text { (LR). }\end{array}$ & $\begin{array}{l}\text { Survey of } \\
\text { inventory } \\
\text { (LR). Best } \\
\text { practice } \\
\text { guidance } \\
\text { (LR). }\end{array}$ & $\begin{array}{l}\text { None } \\
\text { identified. }\end{array}$ & $\begin{array}{l}\text { Best } \\
\text { practice } \\
\text { guidance } \\
\text { (LR). }\end{array}$ & $\begin{array}{l}\text { Survey of } \\
\text { inventory } \\
\text { (LR). }\end{array}$ & $\begin{array}{l}\text { Best practice } \\
\text { guidance } \\
\text { (LR). }\end{array}$ \\
\hline
\end{tabular}


TABLE 3-3-4 Support Goal: Decontamination (gross and final) for Scenario 4

\begin{tabular}{|c|c|c|c|c|c|c|c|c|c|c|c|}
\hline \multirow{2}{*}{$\begin{array}{c}\text { Scenario } \\
\text { Description }\end{array}$} & \multirow{2}{*}{ Summary } & \multirow{2}{*}{ Category } & \multicolumn{9}{|c|}{ Column Number } \\
\hline & & & 1 & 2 & 3 & 4 & 5 & 6 & 7 & 8 & 9 \\
\hline \multirow{5}{*}{$\begin{array}{l}\text { Scenario 4: } \\
\text { As in Scenario 3, } \\
\text { prior studies and } \\
\text { experience show } \\
\text { that } \\
\text { contamination on } \\
\text { paved surfaces } \\
\text { can be effectively } \\
\text { reduced by } \\
\text { washing these } \\
\text { surfaces with } \\
\text { water-based } \\
\text { solutions. What } \\
\text { type of equipment } \\
\text { can wash many } \\
\text { linear miles of } \\
\text { these paved } \\
\text { surfaces and } \\
\text { collect the } \\
\text { washings? }\end{array}$} & \multirow{5}{*}{$\begin{array}{l}\text { - Common } \\
\text { unresolved issues } \\
\text { are: (1) Availability } \\
\text { of privately-owned } \\
\text { equipment. It may } \\
\text { already be in } \\
\text { commercial or } \\
\text { private use, or } \\
\text { owners may be } \\
\text { reluctant to allow its } \\
\text { use in a radioactive } \\
\text { environment } \\
\text { without prior } \\
\text { agreements in place. } \\
\text { (2) Ability to } \\
\text { decontaminate } \\
\text { equipment } \\
\text { afterward for } \\
\text { unrestricted use. } \\
\text { Street sweepers are } \\
\text { attractive options, } \\
\text { but they were not } \\
\text { designed to wash } \\
\text { the street or collect } \\
\text { very small particles. } \\
\text { This makes their } \\
\text { utility dubious. } \\
\text { Studies are } \\
\text { necessary to } \\
\text { determine efficacy } \\
\text { for urban } \\
\text { decontamination. } \\
\text { With any method } \\
\text { that employs water, } \\
\text { controlling runoff } \\
\text { (by diverting it from } \\
\text { sewer inlets or } \\
\text { covering grates) is } \\
\text { important and } \\
\text { difficult, especially } \\
\text { when dumping } \\
\text { thousands of gallons } \\
\text { of water on an area. }\end{array}$} & EQUIPMENT & Mini sweeper. & Street sweeper. & $\begin{array}{l}\text { Large area } \\
\text { pressure } \\
\text { washing. }\end{array}$ & Pressure washing. & Spill barriers/bunds/berms. & $\begin{array}{l}\text { Drain covers or } \\
\text { diverters. }\end{array}$ & Agricultural aircraft. & $\begin{array}{l}\text { Firefighting aircraft } \\
\text { dumpers. }\end{array}$ & Bambi buckets. \\
\hline & & DESCRIPTION & $\begin{array}{l}\text { Smaller scale } \\
\text { street sweeper. }\end{array}$ & $\begin{array}{l}\text { Used to clean dirt, } \\
\text { mud, gravel, and } \\
\text { small debris from } \\
\text { roadways. }\end{array}$ & $\begin{array}{l}\text { Designed to } \\
\text { clean paved } \\
\text { areas. }\end{array}$ & $\begin{array}{l}\text { Common mobile } \\
\text { sprayer (less than } \\
4000 \text { psi), gas or } \\
\text { electric powered, can } \\
\text { provide chemical and } \\
\text { physical removal } \\
\text { mechanisms for } \\
\text { contamination. }\end{array}$ & $\begin{array}{l}\text { Flexible temporary berms to } \\
\text { protect drains, building } \\
\text { entryways, and equipment } \\
\text { from shallow water. }\end{array}$ & $\begin{array}{l}\text { Specialty or ad hoc } \\
\text { covers can protect } \\
\text { intakes or } \\
\text { penetrations from } \\
\text { contaminated water. }\end{array}$ & $\begin{array}{l}\text { Crop dusters or top } \\
\text { spreaders spread } \\
\text { pesticides or } \\
\text { fertilizers over large } \\
\text { tracts of land. } \\
\text { They can similarly } \\
\text { spread water-based } \\
\text { solutions. }\end{array}$ & $\begin{array}{l}\text { Designed to fight } \\
\text { fires by deluging an } \\
\text { area with water from } \\
\text { a nearby water } \\
\text { source. }\end{array}$ & $\begin{array}{l}\text { Designed to fight } \\
\text { fires by deluging an } \\
\text { area with water from } \\
\text { a nearby water } \\
\text { source. }\end{array}$ \\
\hline & & ADVANTAGES & $\begin{array}{l}\text { Water or } \\
\text { waterless } \\
\text { vacuum } \\
\text { sweeper. }\end{array}$ & $\begin{array}{l}\text { Water or waterless } \\
\text { vacuum sweeper. }\end{array}$ & $\begin{array}{l}50 \text { liters per } \\
\text { minute, } 1,650- \\
\text { liter tank. }\end{array}$ & $\begin{array}{l}\text { Large quantities } \\
\text { available COTS, } \\
\text { variable pressure (about } \\
10 \text { liters per minute). }\end{array}$ & $\begin{array}{l}\text { Large quantities available } \\
\text { COTS, flexible material for } \\
\text { custom applications. }\end{array}$ & $\begin{array}{l}\text { COTS (plugs) or } \\
\text { existing infrastructure } \\
\text { (diverters), ad hoc } \\
\text { materials (wood, } \\
\text { polymer sheeting) } \\
\text { likely available. }\end{array}$ & $\begin{array}{l}\text { Large coverage rate, } \\
\text { versatile aircraft. }\end{array}$ & $\begin{array}{l}\text { Can treat large areas, } \\
\text { large volumes } \\
\text { (supertanker: } 20,000 \\
\text { U.S. gallons). }\end{array}$ & $\begin{array}{l}\text { Large volume } \\
\text { capacity (up to } 2600 \\
\text { gallons). }\end{array}$ \\
\hline & & LIMITATIONS & $\begin{array}{l}\text { Designed for } \\
\text { sand and grit } \\
\text { type debris. } \\
\text { Some units do } \\
\text { not have HEPA } \\
\text { filters. }\end{array}$ & $\begin{array}{l}\text { Designed for sand } \\
\text { and grit type } \\
\text { debris. Some units } \\
\text { do not have } \\
\text { HEPA filters. }\end{array}$ & $\begin{array}{l}\text { Secondary waste, } \\
\text { water recycle cab } \\
\text { needed. }\end{array}$ & $\begin{array}{l}\text { Secondary waste, } \\
\text { higher pounds per } \\
\text { square inch units are } \\
\text { limited quantity, small } \\
\text { coverage rate. }\end{array}$ & Shallow waters. & $\begin{array}{l}\text { Large number of } \\
\text { intakes or } \\
\text { penetrations may } \\
\text { require custom } \\
\text { covers. }\end{array}$ & $\begin{array}{l}\text { Amount of water may } \\
\text { be insufficient for } \\
\text { goal. Requires trained } \\
\text { personnel. } \\
\text { Containment of } \\
\text { water, access to urban } \\
\text { canyons, availability }\end{array}$ & $\begin{array}{l}\text { Gross spreader, } \\
\text { availability. Requires } \\
\text { trained personnel. } \\
\text { The impact of water } \\
\text { dropped at elevation } \\
\text { can damage } \\
\text { structures. }\end{array}$ & $\begin{array}{l}\text { Gross spreader, } \\
\text { availability. Requires } \\
\text { trained personnel. } \\
\text { The impact of water } \\
\text { dropped at elevation } \\
\text { can damage } \\
\text { structures. }\end{array}$ \\
\hline & & R\&D NEEDS & $\begin{array}{l}\text { Effectiveness } \\
\text { on smaller } \\
\text { fallout particles } \\
\text { (BE, PE). Water } \\
\text { recycle options } \\
\text { (LR, BE, PE). }\end{array}$ & $\begin{array}{l}\text { Effectiveness on } \\
\text { smaller fallout } \\
\text { particles (BE, PE). } \\
\text { Water recycle } \\
\text { options (LR, BE, } \\
\text { PE). }\end{array}$ & $\begin{array}{l}\text { Survey of } \\
\text { inventory (LR). } \\
\text { Water collection } \\
\text { options (LR). }\end{array}$ & $\begin{array}{l}\text { Data from Fukushima } \\
\text { experience (LR). Best } \\
\text { practice guidance (LR). }\end{array}$ & Best practice guidance (LR). & $\begin{array}{l}\text { Best practice } \\
\text { guidance (LR). }\end{array}$ & $\begin{array}{l}\text { Survey of inventory } \\
\text { (LR). } \\
\text { Guidance on use (LR, } \\
\text { BE, PE). }\end{array}$ & $\begin{array}{l}\text { Survey of inventory } \\
\text { (LR). Guidance on } \\
\text { use (LR, BE, PE). }\end{array}$ & $\begin{array}{l}\text { Guidance on use (LR, } \\
\text { BE, PE). } \\
\text { Survey of inventory } \\
\text { (LR). }\end{array}$ \\
\hline
\end{tabular}

All images are courtesy of Shutterstock, except: (a) permission granted from Ultratech. 
TABLE 3-3-5 Support Goal: Decontamination (gross and final) for Scenario 5

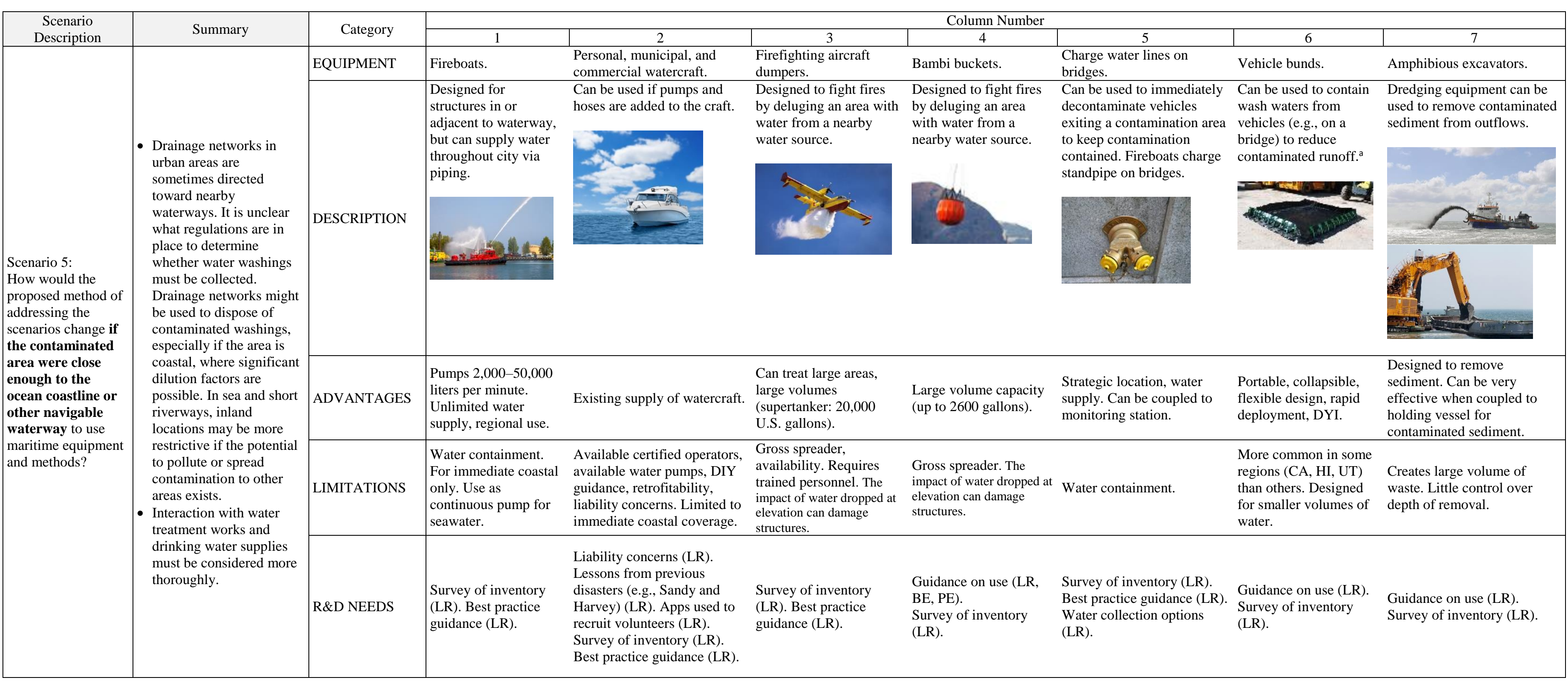


TABLE 3-3-6 Support Goal: Decontamination (gross and final) for Scenario 6

\begin{tabular}{|c|c|c|c|c|c|c|c|}
\hline Scenario 6 & \multicolumn{7}{|c|}{ Responses } \\
\hline $\begin{array}{l}\text { Question to participants: } \\
\text { Do you have other } \\
\text { thoughts or specific } \\
\text { questions based on your } \\
\text { experiences in your } \\
\text { geographical area related } \\
\text { to this topic? }\end{array}$ & $\begin{array}{l}\text { Top-down approach where } \\
\text { workers clean building sides } \\
\text { and roofs first, if needed, using } \\
\text { water for large structures but } \\
\text { with aerosol cross- } \\
\text { contamination in mind. }\end{array}$ & $\begin{array}{l}\text { Not all contaminants are easily } \\
\text { removed using water. The use } \\
\text { of water, with its associated } \\
\text { generation of significant } \\
\text { volumes of secondary waste, } \\
\text { may not be the best option in } \\
\text { many situations. Collection of } \\
\text { water washings is often very } \\
\text { difficult despite best efforts. }\end{array}$ & $\begin{array}{l}\text { The weather could play a } \\
\text { major factor in determining } \\
\text { where the activity goes. In } \\
\text { addition, access restrictions } \\
\text { due to abandoned vehicles } \\
\text { could cause problems. }\end{array}$ & $\begin{array}{l}\text { Public outcry from } \\
\text { defoliation. Need } \\
\text { information campaign to } \\
\text { inform public of activity. }\end{array}$ & $\begin{array}{l}\text { How do we start decontamin- } \\
\text { ation, and where? Liberty } \\
\text { RadEx reported importance } \\
\text { of stakeholder involvement in } \\
\text { decontamination strategy. }\end{array}$ & $\begin{array}{l}\text { Obtaining permission to } \\
\text { enter private residences. } \\
\text { May need to track the } \\
\text { evacuees and obtain } \\
\text { permission to enter their } \\
\text { premises to monitor or } \\
\text { perform decontamination. }\end{array}$ & $\begin{array}{l}\text { Timing is important. If you have } \\
\text { broken communities, not coming } \\
\text { back after } 6 \text { months or so, } \\
\text { consider rebuilding vs. cleaning. } \\
\text { Assess whether it might be better } \\
\text { to turn over the land to developers } \\
\text { and let them redevelop into green } \\
\text { space, for example. However, this } \\
\text { will generate increased amounts } \\
\text { of waste. }\end{array}$ \\
\hline
\end{tabular}

TABLE 3-3-7 Support Goal Training: Decontamination (gross and final) for Scenarios 1-6

\begin{tabular}{|c|c|c|c|c|c|c|c|}
\hline $\begin{array}{l}\text { Support Goal Training: } \\
\text { Scenarios 1-6 }\end{array}$ & Summary & & & Respons & & & \\
\hline $\begin{array}{l}\text { Question to participants: } \\
\text { What are your thoughts and } \\
\text { recommendations on the } \\
\text { availability of trained } \\
\text { human assets and training } \\
\text { of the additional assets } \\
\text { likely needed to accomplish } \\
\text { the scenarios under this goal? } \\
\text { (It is understood that training } \\
\text { will be a significant effort } \\
\text { and an additional limiting } \\
\text { factor in any response } \\
\text { scenario. Further, it may need } \\
\text { to be addressed more } \\
\text { thoroughly in the future, but } \\
\text { input is needed to help guide } \\
\text { how training guidance should } \\
\text { be developed.) }\end{array}$ & $\begin{array}{l}\text { Wide-area radiological } \\
\text { contamination incidents are } \\
\text { rare, and a response to such } \\
\text { an incident will require } \\
\text { tremendous human assets. } \\
\text { Lessons learned from the } \\
\text { cleanup efforts in Japan } \\
\text { showed that many thousands } \\
\text { of individuals each day are } \\
\text { engaged in clean-up } \\
\text { activities. }\end{array}$ & $\begin{array}{l}\text { Such decontamination } \\
\text { responses require a unified } \\
\text { effort with a central chain of } \\
\text { command. This is essential to } \\
\text { ensure safe decontamination of } \\
\text { adjacent areas, and to prevent } \\
\text { re-contamination of previously } \\
\text { decontaminated areas. }\end{array}$ & $\begin{array}{l}\text { It is also essential that clear } \\
\text { objectives be set; a defined end } \\
\text { point or "clean-down level" must } \\
\text { be decided upon for each location, } \\
\text { surface, etc., prior to the start of } \\
\text { decontamination. Any } \\
\text { remediation or decontamination } \\
\text { would need to be legally } \\
\text { defensible by the supervising } \\
\text { authority. }\end{array}$ & $\begin{array}{l}\text { The Nuclear Regulatory } \\
\text { Commission and DOE have } \\
\text { training programs for work with } \\
\text { radioactive materials. The } \\
\text { Environmental Protection Agency } \\
\text { has Hazardous Waste Operations } \\
\text { and Emergency Response Standard } \\
\text { (HAZ-WOPER) for contractors } \\
\text { and workers to receive a 40-hour } \\
\text { OSHA course that will cover PPE, } \\
\text { hazard, and working in a } \\
\text { contaminated environment. Then, } \\
\text { they receive basic and advanced } \\
\text { radiation safety training (as } \\
\text { appropriate), and respirator use and } \\
\text { care if they use one. }\end{array}$ & $\begin{array}{l}\text { Decontamination of small } \\
\text { plots of land may be more } \\
\text { cost effective and achievable } \\
\text { if undertaken by landowners. } \\
\text { Minimal training is required } \\
\text { to use a shovel, although } \\
\text { respiratory protection against } \\
\text { resuspension of material and } \\
\text { suitable waste disposal } \\
\text { measures would clearly need } \\
\text { to be considered as part of } \\
\text { any training. }\end{array}$ & $\begin{array}{l}\text { SQEP individuals with } \\
\text { radiological experience would } \\
\text { be in short supply. Add in the } \\
\text { complexities of remediation in } \\
\text { unfamiliar working } \\
\text { environments, and there are } \\
\text { significant conventional and } \\
\text { radiological hazards to be } \\
\text { aware. }\end{array}$ & $\begin{array}{l}\text { Providing basic radiological } \\
\text { training for specialist operators } \\
\text { with no radiological experience } \\
\text { is likely to be the most cost } \\
\text { effective and timely option for } \\
\text { ensuring SQEP workers are used } \\
\text { on site. Teams would include } \\
\text { trained health physicists or } \\
\text { monitors to ensure radiological } \\
\text { safety of all staff on site. This is } \\
\text { common practice on non-nuclear } \\
\text { sites for remediation work. }\end{array}$ \\
\hline
\end{tabular}




\subsection{SUPPORT GOAL: WASTE MANAGEMENT}

Businesses and residences over a wide area will generate contaminated solid waste in varying sizes and container types. Solid radioactive waste should be collected for staging and disposal. What types of municipal and commercial equipment can stabilize, contain, store, and transport the radioactive solid waste generated during mitigation and decontamination operations? Examples include using municipal waste garbage trucks and current routes to pick up garbage associated with small-scale (local) operations, and using existing software and procedures developed for the transport of radioactive material to identify preferred routes. Tables 3-4-1 to 3-4-5 present various scenarios related to waste management, possible responses to the scenarios, and possible equipment/technology used in responding, including its advantages, limitations, and $\mathrm{R} \& \mathrm{D}$ needs. 
TABLE 3-4-1 Support Goal: Waste Management for Scenario 1

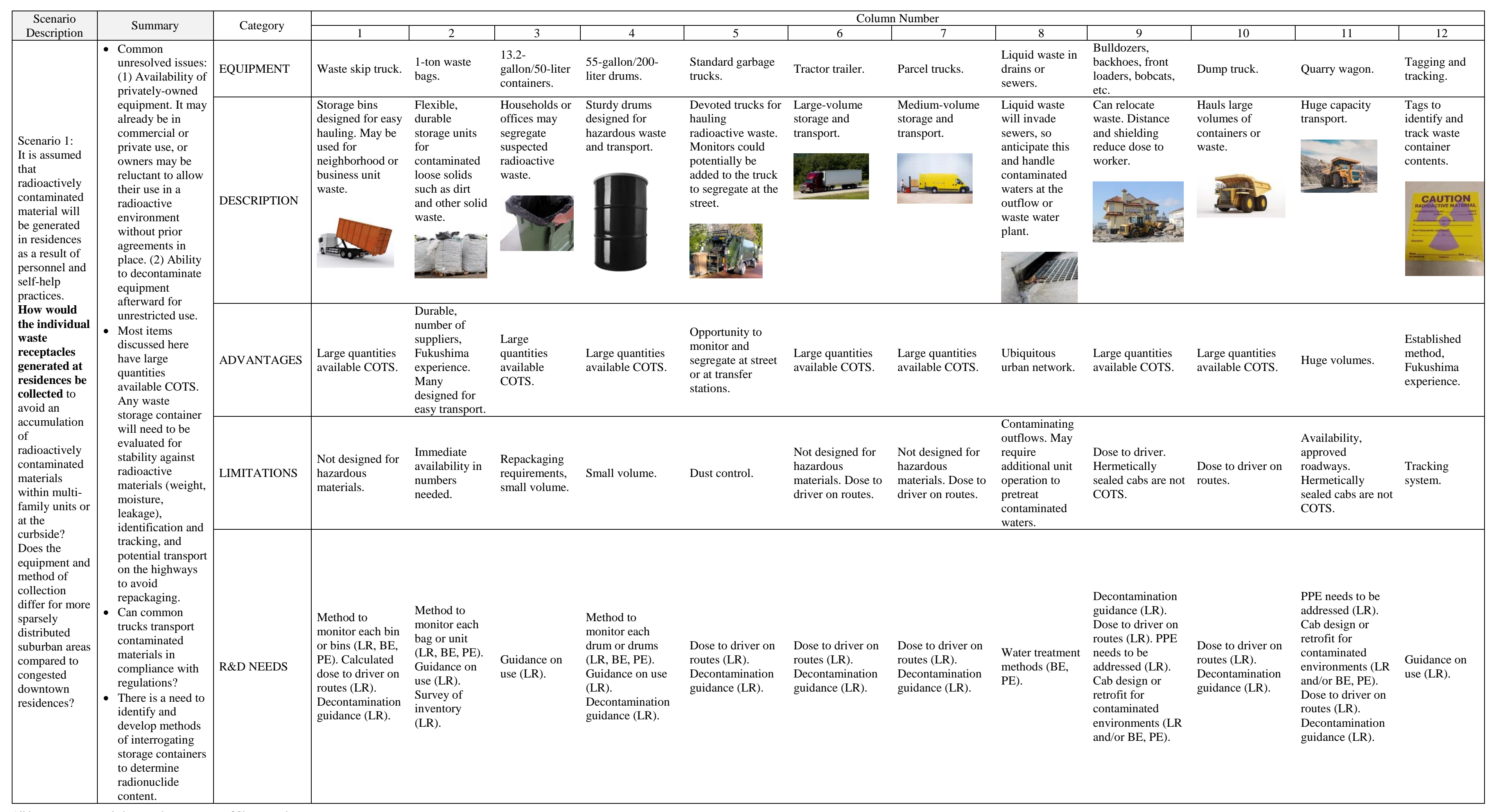

All imges are persont photegne or courtesy of Shuterstock 
TABLE 3-4-2 Support Goal: Waste Management for Scenario 2

\begin{tabular}{|c|c|c|c|c|c|c|c|c|c|c|c|c|}
\hline \multirow{2}{*}{$\begin{array}{c}\text { Scenario } \\
\text { Description }\end{array}$} & \multirow{2}{*}{ Summary } & \multirow{2}{*}{ Category } & \multicolumn{10}{|c|}{ Column Number } \\
\hline & & & 1 & 2 & 3 & 4 & 5 & 6 & 7 & 8 & 9 & 10 \\
\hline \multirow{5}{*}{$\begin{array}{l}\text { Scenario 2: } \\
\text { How would the } \\
\text { response to } \\
\text { Scenario } 1 \text { differ if } \\
\text { the radiaactively } \\
\text { contaminated } \\
\text { material is } \\
\text { generated by local } \\
\text { businesses a a } \\
\text { result of self-help } \\
\text { decontamination? } \\
\text { How would the } \\
\text { individual waste } \\
\text { receptacles } \\
\text { generated by local } \\
\text { businesses be } \\
\text { collected to avoid } \\
\text { an accumulation of } \\
\text { radioactively } \\
\text { contaminated } \\
\text { materials in multi- } \\
\text { business units or } \\
\text { curbside? Do the } \\
\text { equipment and } \\
\text { method of } \\
\text { collection differ } \\
\text { for more sparsely } \\
\text { distributed } \\
\text { suburban areas } \\
\text { compared to } \\
\text { congested } \\
\text { downtown } \\
\text { businesses? }\end{array}$} & \multirow{5}{*}{$\begin{array}{l}\text { - Many items discussed } \\
\text { in Scenario } 1 \text { also } \\
\text { apply here } \\
\text { (availability, } \\
\text { unrestricted use after } \\
\text { event, transport } \\
\text { regulations, monitoring } \\
\text { package). } \\
\text { - There may be better } \\
\text { opportunity for } \\
\text { businesses to follow } \\
\text { recommended practices } \\
\text { to segregate and } \\
\text { package waste to avoid } \\
\text { additional effort. That } \\
\text { is, businesses can be } \\
\text { more easily } \\
\text { incentivized and held } \\
\text { accountable for correct } \\
\text { disposal of such waste } \\
\text { than individuals. } \\
\text { However, it is also } \\
\text { more likely that } \\
\text { businesses will hire } \\
\text { contractors to do the } \\
\text { work. Businesses tend } \\
\text { to have large waste } \\
\text { containers that could } \\
\text { be used with the } \\
\text { frequency of collection } \\
\text { increased. } \\
\text { - Need to consider } \\
\text { approach of collecting } \\
\text { all garbage as usual } \\
\text { and monitoring or } \\
\text { segregating } \\
\text { contaminated garbage } \\
\text { per existing procedures } \\
\text { at transfer stations and } \\
\text { waste sites. } \\
\text { - There is a need to } \\
\text { identify and develop } \\
\text { methods of } \\
\text { interrogating storage } \\
\text { containers to determine } \\
\text { radionuclide content. }\end{array}$} & EQUIPMENT & $\begin{array}{l}\text { Waste skip } \\
\text { truck. }\end{array}$ & $\begin{array}{l}\text { 1-ton waste } \\
\text { bags. }\end{array}$ & $\begin{array}{l}\text { 13.2-gallon/ } \\
\text { 50-liter } \\
\text { containers. }\end{array}$ & $\begin{array}{l}\text { 55-gallon/ } \\
200 \text {-liter drums. }\end{array}$ & $\begin{array}{l}\text { Standard } \\
\text { garbage trucks. }\end{array}$ & Tractor trailer. & Parcel trucks. & $\begin{array}{l}\text { Liquid waste } \\
\text { in drains or } \\
\text { sewers. }\end{array}$ & Dump truck. & Quarry wagon. \\
\hline & & DESCRIPTION & $\begin{array}{l}\text { Storage bins } \\
\text { designed for } \\
\text { easy hauling. } \\
\text { May be used for } \\
\text { neighborhood or } \\
\text { business unit } \\
\text { waste. }\end{array}$ & $\begin{array}{l}\text { Flexible, durable } \\
\text { storage units for } \\
\text { contaminated } \\
\text { loose solids such } \\
\text { as dirt and other } \\
\text { solid waste. }\end{array}$ & $\begin{array}{l}\text { Households or } \\
\text { offices may } \\
\text { segregate } \\
\text { suspected } \\
\text { radioactive } \\
\text { waste. }\end{array}$ & $\begin{array}{l}\text { Sturdy drums } \\
\text { designed for } \\
\text { hazardous waste } \\
\text { and transport. }\end{array}$ & $\begin{array}{l}\text { Devoted trucks } \\
\text { for hauling } \\
\text { radioactive } \\
\text { waste. Monitors } \\
\text { could potentially } \\
\text { be added to the } \\
\text { truck to } \\
\text { segregate at the } \\
\text { street. }\end{array}$ & $\begin{array}{l}\text { Large-volume } \\
\text { storage and } \\
\text { transport. }\end{array}$ & $\begin{array}{l}\text { Medium-volume } \\
\text { storage and } \\
\text { transport. }\end{array}$ & $\begin{array}{l}\text { Liquid waste } \\
\text { will get into } \\
\text { sewers, so } \\
\text { perhaps design } \\
\text { unit operation } \\
\text { to anticipate } \\
\text { this and } \\
\text { handle } \\
\text { contaminated } \\
\text { waters at the } \\
\text { plant. }\end{array}$ & $\begin{array}{l}\text { Hauls large } \\
\text { volumes of } \\
\text { containers or } \\
\text { waste. }\end{array}$ & $\begin{array}{l}\text { Huge capacity } \\
\text { transport. }\end{array}$ \\
\hline & & ADVANTAGES & $\begin{array}{l}\text { Large quantities } \\
\text { available COTS. }\end{array}$ & $\begin{array}{l}\text { Durable, number } \\
\text { of suppliers, } \\
\text { Fukushima } \\
\text { experience. } \\
\text { Many designed } \\
\text { for easy } \\
\text { transport. }\end{array}$ & $\begin{array}{l}\text { Large quantities } \\
\text { available } \\
\text { COTS. }\end{array}$ & $\begin{array}{l}\text { Large quantities } \\
\text { available COTS. }\end{array}$ & $\begin{array}{l}\text { Opportunity to } \\
\text { monitor and } \\
\text { segregate at } \\
\text { street or at } \\
\text { transfer stations. }\end{array}$ & $\begin{array}{l}\text { Large quantities } \\
\text { available COTS. }\end{array}$ & $\begin{array}{l}\text { Large quantities } \\
\text { available COTS. }\end{array}$ & $\begin{array}{l}\text { Ubiquitous } \\
\text { urban } \\
\text { network. }\end{array}$ & $\begin{array}{l}\text { Large quantities } \\
\text { available COTS. }\end{array}$ & Huge volumes. \\
\hline & & LIMITATIONS & $\begin{array}{l}\text { Not designed for } \\
\text { hazardous } \\
\text { materials. }\end{array}$ & $\begin{array}{l}\text { Immediate } \\
\text { availability in } \\
\text { numbers needed. }\end{array}$ & $\begin{array}{l}\text { Repackaging } \\
\text { requirements, } \\
\text { small volume. }\end{array}$ & Small volume. & Dust control. & $\begin{array}{l}\text { Not designed for } \\
\text { hazardous } \\
\text { materials. Dose to } \\
\text { driver on routes. }\end{array}$ & $\begin{array}{l}\text { Not designed for } \\
\text { hazardous } \\
\text { materials. Dose to } \\
\text { driver on routes. }\end{array}$ & $\begin{array}{l}\text { Contaminating } \\
\text { outflows. }\end{array}$ & $\begin{array}{l}\text { Dose to driver } \\
\text { on routes. }\end{array}$ & $\begin{array}{l}\text { Availability, } \\
\text { approved } \\
\text { roadways. } \\
\text { Hermetically } \\
\text { sealed cabs are } \\
\text { not COTS. } \\
\end{array}$ \\
\hline & & R\&D NEEDS & $\begin{array}{l}\text { Method to } \\
\text { monitor each bin } \\
\text { or bins (LR, BE, } \\
\text { PE). Calculated } \\
\text { dose to driver on } \\
\text { routes (LR). } \\
\text { Decontamination } \\
\text { guidance (LR). }\end{array}$ & $\begin{array}{l}\text { Method to } \\
\text { monitor each bag } \\
\text { or unit (LR, BE, } \\
\text { PE). Guidance } \\
\text { on use (LR). } \\
\text { Survey of } \\
\text { inventory (LR). }\end{array}$ & $\begin{array}{l}\text { Guidance on } \\
\text { use (LR). }\end{array}$ & $\begin{array}{l}\text { Method to } \\
\text { monitor each } \\
\text { drum or drums } \\
\text { (LR, BE, PE). } \\
\text { Guidance on use } \\
\text { (LR). } \\
\text { Decontamination } \\
\text { guidance (LR). }\end{array}$ & $\begin{array}{l}\text { Dose to driver } \\
\text { on routes (LR). } \\
\text { Decontamination } \\
\text { guidance (LR). }\end{array}$ & $\begin{array}{l}\text { Dose to driver } \\
\text { on routes (LR). } \\
\text { Decontamination } \\
\text { guidance (LR). }\end{array}$ & $\begin{array}{l}\text { Dose to driver } \\
\text { on routes (LR). } \\
\text { Decontamination } \\
\text { guidance (LR). }\end{array}$ & $\begin{array}{l}\text { Water } \\
\text { treatment } \\
\text { methods (BE, } \\
\text { PE). }\end{array}$ & $\begin{array}{l}\text { Dose to driver } \\
\text { on routes (LR). } \\
\text { Decontamination } \\
\text { guidance (LR). }\end{array}$ & $\begin{array}{l}\text { PPE needs to be } \\
\text { addressed (LR). } \\
\text { Cab design or } \\
\text { retrofit for } \\
\text { contaminated } \\
\text { environments } \\
\text { (LR and/or BE, } \\
\text { PE). Dose to } \\
\text { driver on routes } \\
\text { (LR). } \\
\text { Decontamination } \\
\text { guidance (LR). }\end{array}$ \\
\hline
\end{tabular}

All images are courtesy of Shutterstock. 
TABLE 3-4-3 Support Goal: Waste Management for Scenario 3

\begin{tabular}{|c|c|c|c|c|c|c|c|c|c|c|c|c|c|}
\hline \multirow{2}{*}{$\begin{array}{c}\text { Scenario } \\
\text { Description }\end{array}$} & \multirow{2}{*}{ Summary } & \multirow{2}{*}{ Category } & \multicolumn{11}{|c|}{ Column Number } \\
\hline & & & 1 & 2 & 3 & 4 & 5 & 6 & 7 & 8 & 9 & 10 & 11 \\
\hline \multirow{5}{*}{$\begin{array}{l}\text { Scenario 3: } \\
\text { If accumulated } \\
\text { radioactively } \\
\text { contaminated } \\
\text { waste is being } \\
\text { collected at } \\
\text { staging locations } \\
\text { (e.g., parks and } \\
\text { common } \\
\text { grounds), what } \\
\text { types of containers } \\
\text { can be used to } \\
\text { collect the waste } \\
\text { receptacles and } \\
\text { protect the public } \\
\text { from transport of } \\
\text { contaminated } \\
\text { particles (e.g., } \\
\text { through } \\
\text { resuspension of } \\
\text { breached } \\
\text { receptacles)? }\end{array}$} & \multirow{5}{*}{$\begin{array}{l}\text { - Common unresolved } \\
\text { issues: (1) } \\
\text { Availability of } \\
\text { privately-owned } \\
\text { equipment. It may } \\
\text { already be in } \\
\text { commercial or private } \\
\text { use, or owners may be } \\
\text { reluctant to allow their } \\
\text { use in a radioactive } \\
\text { environment without } \\
\text { prior agreements in } \\
\text { place. (2) Ability to } \\
\text { decontaminate } \\
\text { equipment afterward } \\
\text { for unrestricted use. } \\
\text { - Estimates of dose to } \\
\text { the public from } \\
\text { nearby staging } \\
\text { locations are needed. } \\
\text { - Security and } \\
\text { ruggedness of } \\
\text { containers to weather } \\
\text { (rain, snow, heat, } \\
\text { ultraviolet light) at } \\
\text { staging locations is an } \\
\text { issue. } \\
\text { - Guidance on how to } \\
\text { predetermine potential } \\
\text { staging locations is } \\
\text { needed. }\end{array}$} & EQUIPMENT & $\begin{array}{l}\text { Freight } \\
\text { containers } \\
\text { (lockable). }\end{array}$ & $\begin{array}{l}\text { Roll-on/roll-off } \\
\text { containers. }\end{array}$ & $\begin{array}{l}55 \text {-gallon/ } \\
200 \text {-liter drums. }\end{array}$ & Large tanks. & Cargo ships. & $\begin{array}{l}\text { 1-ton waste } \\
\text { bags. }\end{array}$ & $\begin{array}{l}\text { Local } \\
\text { premises. }\end{array}$ & $\begin{array}{l}\text { Intermediate } \\
\text { bulk containers } \\
\text { (IBCs). }\end{array}$ & Barges. & $\begin{array}{l}\text { Cardboard } \\
\text { boxes. }\end{array}$ & $\begin{array}{l}\text { Temporary } \\
\text { lagoons. }\end{array}$ \\
\hline & & DESCRIPTION & $\begin{array}{l}\text { Covered and } \\
\text { transportable } \\
\text { containers. }\end{array}$ & $\begin{array}{l}\text { Covered and } \\
\text { transportable } \\
\text { containers. }\end{array}$ & $\begin{array}{l}\text { Sturdy drums } \\
\text { designed for } \\
\text { hazardous waste } \\
\text { and transport. }\end{array}$ & $\begin{array}{l}\text { Any number } \\
\text { of tank sizes } \\
\text { available or } \\
\text { in use for } \\
\text { storage. }\end{array}$ & Huge capacity & $\begin{array}{l}\text { Flexible, } \\
\text { durable } \\
\text { storage units } \\
\text { for } \\
\text { contaminated } \\
\text { loose solids } \\
\text { such as dirt } \\
\text { and other } \\
\text { solid waste. }\end{array}$ & $\begin{array}{l}\text { Town halls, } \\
\text { sports } \\
\text { stadiums, } \\
\text { postal depots, } \\
\text { etc., may be } \\
\text { used to stage } \\
\text { waste. }\end{array}$ & $\begin{array}{l}\text { Medium-sized } \\
\text { container for } \\
\text { liquids. }\end{array}$ & $\begin{array}{l}\text { Huge } \\
\text { capacity for } \\
\text { storage and } \\
\text { transport. }\end{array}$ & $\begin{array}{l}\text { Affordable } \\
\text { container for } \\
\text { dry, blunt, } \\
\text { rigid objects. }\end{array}$ & $\begin{array}{l}\text { Huge capacity } \\
\text { for liquid } \\
\text { storage. }\end{array}$ \\
\hline & & ADVANTAGES & $\begin{array}{l}\text { Large quantities } \\
\text { available COTS. }\end{array}$ & $\begin{array}{l}\text { Large } \\
\text { quantities } \\
\text { available } \\
\text { COTS. }\end{array}$ & $\begin{array}{l}\text { Large quantities } \\
\text { available COTS. }\end{array}$ & $\begin{array}{l}\text { Huge } \\
\text { capacity. }\end{array}$ & $\begin{array}{l}\text { Huge } \\
\text { capacity. }\end{array}$ & $\begin{array}{l}\text { Durable, a } \\
\text { number of } \\
\text { suppliers, } \\
\text { Fukushima } \\
\text { experience. } \\
\text { Many } \\
\text { designed for } \\
\text { easy } \\
\text { transport. }\end{array}$ & $\begin{array}{l}\text { Local use, } \\
\text { large } \\
\text { quantities } \\
\text { available, } \\
\text { network. }\end{array}$ & $\begin{array}{l}\text { Mass produced, } \\
\text { easily } \\
\text { transported, } \\
\text { easily stacked. } \\
\text { Compatible } \\
\text { with pumps and } \\
\text { mixers. } \\
\text { Fukushima } \\
\text { experience. }\end{array}$ & $\begin{array}{l}\text { Huge } \\
\text { capacity. }\end{array}$ & $\begin{array}{l}\text { Large } \\
\text { quantities } \\
\text { available } \\
\text { COTS. }\end{array}$ & $\begin{array}{l}\text { Common to } \\
\text { hydraulic } \\
\text { fracturing, } \\
\text { evaporation. }\end{array}$ \\
\hline & & LIMITATIONS & $\begin{array}{l}\text { Not designed for } \\
\text { hazardous } \\
\text { materials. }\end{array}$ & $\begin{array}{l}\text { Not designed } \\
\text { for hazardous } \\
\text { materials. }\end{array}$ & Small volume. & $\begin{array}{l}\text { Availability, } \\
\text { onsite } \\
\text { construction } \\
\text { period. }\end{array}$ & Availability. & $\begin{array}{l}\text { Immediate } \\
\text { availability } \\
\text { in numbers } \\
\text { needed. }\end{array}$ & $\begin{array}{l}\text { Security, } \\
\text { public } \\
\text { perception. }\end{array}$ & $\begin{array}{l}\text { Designed for } \\
\text { liquid waste. }\end{array}$ & $\begin{array}{l}\text { Few plans on } \\
\text { eventual } \\
\text { destination. }\end{array}$ & $\begin{array}{l}\text { Not rugged or } \\
\text { secure. }\end{array}$ & Attract wildlife. \\
\hline & & R\&D NEEDS & $\begin{array}{l}\text { Method to } \\
\text { monitor each bin } \\
\text { or container (LR, } \\
\text { BE, PE). } \\
\text { Calculated dose } \\
\text { to driver on } \\
\text { routes (LR). } \\
\text { Decontamination } \\
\text { guidance (LR). }\end{array}$ & $\begin{array}{l}\text { Method to } \\
\text { monitor each } \\
\text { bin or } \\
\text { container (LR, } \\
\text { BE, PE). } \\
\text { Calculated } \\
\text { dose to driver } \\
\text { on routes (LR). } \\
\text { Decontaminati } \\
\text { on guidance } \\
\text { (LR). }\end{array}$ & $\begin{array}{l}\text { Method to } \\
\text { monitor each } \\
\text { drum or drums } \\
\text { (LR, BE, PE). } \\
\text { Guidance on use } \\
\text { (LR). } \\
\text { Decontamination } \\
\text { guidance (LR). }\end{array}$ & $\begin{array}{l}\text { Survey of } \\
\text { inventory } \\
\text { (LR). }\end{array}$ & $\begin{array}{l}\text { Survey of } \\
\text { inventory } \\
\text { (LR). }\end{array}$ & $\begin{array}{l}\text { Method to } \\
\text { monitor each } \\
\text { bin or bins } \\
\text { (LR, BE, } \\
\text { PE). } \\
\text { Guidance on } \\
\text { use (LR). }\end{array}$ & $\begin{array}{l}\text { Guidance on } \\
\text { use (LR). }\end{array}$ & $\begin{array}{l}\text { Method to } \\
\text { monitor each } \\
\text { bin or bins (LR, } \\
\text { BE, PE). } \\
\text { Guidance on } \\
\text { use (LR). }\end{array}$ & $\begin{array}{l}\text { Survey of } \\
\text { inventory } \\
\text { (LR). }\end{array}$ & $\begin{array}{l}\text { Guidance on } \\
\text { use (LR). }\end{array}$ & $\begin{array}{l}\text { Survey of } \\
\text { inventory (LR). } \\
\text { Guidance on } \\
\text { use (LR). }\end{array}$ \\
\hline
\end{tabular}

All images are courtesy of Shutterstock, except: (a) permission granted from ModuTank, Inc. 
TABLE 3-4-4 Support Goal: Waste Management for Scenario 4

\begin{tabular}{|c|c|c|c|c|c|c|c|}
\hline Scenario 4 & \multicolumn{7}{|c|}{ Responses } \\
\hline $\begin{array}{l}\text { Question to participants: } \\
\text { Do you have other } \\
\text { thoughts or specific } \\
\text { questions based on your } \\
\text { experiences in your } \\
\text { geographical area related } \\
\text { to this topic? }\end{array}$ & $\begin{array}{l}\text { It is difficult to } \\
\text { envision a scenario, } \\
\text { where a family or the } \\
\text { public will be present } \\
\text { in an area, if the } \\
\text { radiation or } \\
\text { contamination levels } \\
\text { are such that their } \\
\text { waste will generate a } \\
\text { significant secondary } \\
\text { hazard to others. }\end{array}$ & $\begin{array}{l}\text { Use normal waste } \\
\text { bins, and then } \\
\text { monitor each } \\
\text { truckload for gross } \\
\text { contamination and } \\
\text { manage that. }\end{array}$ & $\begin{array}{l}\text { Apartment and multi- } \\
\text { family unit residents } \\
\text { would probably be more } \\
\text { reluctant to participate in } \\
\text { cleanup work, and would } \\
\text { instead rely on apartment } \\
\text { managers and property } \\
\text { owners to perform the } \\
\text { work. }\end{array}$ & $\begin{array}{l}\text { Monitor garbage at landfill } \\
\text { entry or transfer site entry } \\
\text { like current garbage and } \\
\text { recycled material. }\end{array}$ & $\begin{array}{l}\text { Not understanding the } \\
\text { waste container } \\
\text { requirements during } \\
\text { generation of waste leads } \\
\text { to an inevitable re-sizing } \\
\text { process to make waste fit } \\
\text { new containers. }\end{array}$ & $\begin{array}{l}\text { Regular garbage or parcel } \\
\text { delivery teams would know the } \\
\text { area, the routes that are } \\
\text { accessible by truck are SQEP } \\
\text { for manual handling, and with } \\
\text { health physics support would } \\
\text { be aware of the radiological } \\
\text { hazards. }\end{array}$ & $\begin{array}{l}\text { We need to understand DOT } \\
\text { regulations for transport } \\
\text { (considering everything } \\
\text { might be contaminated). If } \\
\text { transport occurs within the } \\
\text { contaminated area, does } \\
\text { DOT regulations matter? }\end{array}$ \\
\hline
\end{tabular}

TABLE 3-4-5 Support Goal Training: Scenarios 1-4

\begin{tabular}{|c|c|c|c|c|c|c|c|}
\hline $\begin{array}{l}\text { Support Goal Training: } \\
\text { Scenarios 1-4 }\end{array}$ & Summary & & & Respo & nses & & \\
\hline $\begin{array}{l}\text { Question to participants: } \\
\text { What are your thoughts } \\
\text { and recommendations } \\
\text { about the availability of } \\
\text { trained human assets } \\
\text { and training additional } \\
\text { assets to accomplish the } \\
\text { scenarios under this } \\
\text { goal? (Training will be a } \\
\text { significant effort and an } \\
\text { additional limiting factor } \\
\text { in any response. It may } \\
\text { need to be addressed } \\
\text { more thoroughly in the } \\
\text { future, but input is } \\
\text { needed to help guide } \\
\text { how training guidance } \\
\text { should be developed.) }\end{array}$ & $\begin{array}{l}\text { Wide-area } \\
\text { radiological } \\
\text { contamination } \\
\text { incidents are rare, } \\
\text { and a response to } \\
\text { such an incident will } \\
\text { require tremendous } \\
\text { human assets. } \\
\text { Lessons learned from } \\
\text { the cleanup efforts in } \\
\text { Japan showed that } \\
\text { many thousands of } \\
\text { individuals each day } \\
\text { are engaged in } \\
\text { cleanup activities. }\end{array}$ & $\begin{array}{l}\text { Legislation requires } \\
\text { employers to ensure } \\
\text { their workforce is } \\
\text { suitably trained for } \\
\text { any tasks they are } \\
\text { required to perform. }\end{array}$ & $\begin{array}{l}\text { Training on waste } \\
\text { minimization and } \\
\text { radiation safety } \\
\text { would allow } \\
\text { members of the } \\
\text { public to make an } \\
\text { effective contribution } \\
\text { to the cleanup } \\
\text { operations. }\end{array}$ & $\begin{array}{l}\text { The Nuclear Regulatory } \\
\text { Commission and DOE have } \\
\text { training programs for work with } \\
\text { radioactive materials. The } \\
\text { Environmental Protection Agency } \\
\text { has HAZWOPER for contractors } \\
\text { and workers to receive a 40-hour } \\
\text { OSHA course that will cover PPE, } \\
\text { hazard, and working in a } \\
\text { contaminated environment. Then, } \\
\text { they receive basic and advanced } \\
\text { training on radiation safety (as } \\
\text { appropriate), and respirator use and } \\
\text { care if they use one. }\end{array}$ & $\begin{array}{l}\text { Waste facilities are } \\
\text { well regulated and } \\
\text { controlled in } \\
\text { comparison to hands- } \\
\text { on decontamination } \\
\text { work. With the right } \\
\text { technical experts and } \\
\text { SQEP operators, a } \\
\text { waste facility is } \\
\text { relatively } \\
\text { straightforward to } \\
\text { operate and involves } \\
\text { limited training of new } \\
\text { staff in unfamiliar } \\
\text { roles. }\end{array}$ & $\begin{array}{l}\text { Running a waste facility is } \\
\text { a complex task involving } \\
\text { multiple experts including } \\
\text { a site manager, radioactive } \\
\text { waste adviser, forklift } \\
\text { operators, HP monitors, a } \\
\text { Dangerous Goods Adviser, } \\
\text { and an Environmental } \\
\text { Consultant. It should be } \\
\text { given equal consideration } \\
\text { to the actual cleanup. }\end{array}$ & $\begin{array}{l}\text { In Nevada, they specifically } \\
\text { registered and marked waste } \\
\text { trucks would haul } \\
\text { contaminated waste to } \\
\text { Nevada National Security } \\
\text { Site Area } 5 \text { for sorting and } \\
\text { disposal. Those drivers and } \\
\text { workers would need basic } \\
\text { radiation protection training, } \\
\text { dosimetry, and PPE. }\end{array}$ \\
\hline
\end{tabular}




\subsection{SUPPORT GOAL: CONTAINMENT OF WASTEWATER AND OTHER WASTES}

First responders will likely use water to extinguish fires that may be generated during a radioactive release. Water may also be used to reduce radiation levels to early responders and subsequent response teams. Ideally, the water could be collected and treated at the point of use. However, we may need to collect, divert, and store generated radioactively contaminated waters for proper treatment and disposal. What types of municipal and commercial equipment can collect, contain, and transport liquid wastes and other wastes generated after a radioactive release? Examples include portable tanks and storage bladders, barges, tanker trucks, railroad tank cars, fixed tank farms such as those at refineries, the storm sewer, and sewer water storage tunnels and reservoirs. Tables 3-5-1 to 3-5-8 present various scenarios related to containment of wastewater and other wastes, possible responses to the scenarios, and possible equipment/technology used in responding, including its advantages, limitations, and R\&D needs. 
TABLE 3-5-1 Support Goal: Containment of Wastewater and Other Wastes for Scenario 1

\begin{tabular}{|c|c|c|c|c|c|c|c|c|c|c|}
\hline \multirow[b]{2}{*}{ Scenario Description } & \multirow[b]{2}{*}{ Summary } & \multirow[b]{2}{*}{ Category } & \multicolumn{8}{|c|}{ Column Number } \\
\hline & & & 1 & 2 & 3 & 4 & 5 & 6 & 7 & 8 \\
\hline \multirow{5}{*}{$\begin{array}{l}\text { Scenario 1: } \\
\text { What types of barriers } \\
\text { (e.g., sandbags and } \\
\text { berms) are available } \\
\text { to collect water } \\
\text { generated during its } \\
\text { large-scale use } \\
\text { (hundreds of thousands } \\
\text { to millions of gallons) } \\
\text { over a small urban } \\
\text { footprint (for example, } \\
\text { a square city block)? }\end{array}$} & \multirow{5}{*}{$\begin{array}{l}\text { - Inevitable leakage of some } \\
\text { waters is a common } \\
\text { problem for these } \\
\text { technologies. } \\
\text { - Many of the items listed } \\
\text { here have large quantities } \\
\text { available COTS. } \\
\text { - Knowledge of the } \\
\text { topography of the terrain } \\
\text { will be essential to contain } \\
\text { large volumes of water. } \\
\text { - Guidance on their specific } \\
\text { use is lacking. } \\
\text { - Although many cities have } \\
\text { GIS sewer maps, guidance } \\
\text { is needed on how to best } \\
\text { apply these in a } \\
\text { radiological contamination } \\
\text { incident to achieve the } \\
\text { goals of containment. } \\
\text { - Knowledge of whether } \\
\text { sewer lines can be } \\
\text { plugged to hold water in } \\
\text { an individual } \\
\text { neighborhood or block is } \\
\text { lacking. }\end{array}$} & EQUIPMENT & $\begin{array}{l}\begin{array}{l}\text { Spill } \\
\text { barriers/bunds/berms. }\end{array} \\
\end{array}$ & $\begin{array}{l}\text { Drain covers or } \\
\text { diverters. }\end{array}$ & Sandbags. & $\begin{array}{l}\text { Flood control } \\
\text { barriers. }\end{array}$ & $\begin{array}{l}\text { Inflatable tube } \\
\text { barriers. }\end{array}$ & $\begin{array}{l}\text { Inflatable pipe and } \\
\text { sewer plugs. }\end{array}$ & $\begin{array}{l}\text { Pumps and } \\
\text { piping. }\end{array}$ & Sewer lines. \\
\hline & & DESCRIPTION & $\begin{array}{l}\text { Flexible temporary } \\
\text { berms to protect } \\
\text { drains, building } \\
\text { entryways, and } \\
\text { equipment from } \\
\text { shallow water. }\end{array}$ & $\begin{array}{l}\text { Specialty or ad } \\
\text { hoc covers can } \\
\text { protect intakes or } \\
\text { penetrations } \\
\text { from } \\
\text { contaminated } \\
\text { water. }^{\text {a }}\end{array}$ & $\begin{array}{l}\text { Effective flood } \\
\text { control achieved by } \\
\text { building a wall of } \\
\text { stacked bags. }\end{array}$ & $\begin{array}{l}\text { Emergency barriers } \\
\text { supplied worldwide } \\
\text { during emergencies } \\
\text { to control and } \\
\text { contain water. }\end{array}$ & $\begin{array}{l}\text { Inflatable PVC tubes } \\
\text { to contain/divert } \\
\text { water. }\end{array}$ & $\begin{array}{l}\text { Inflatable plugs for } \\
\text { plugging sewer } \\
\text { lines. }\end{array}$ & $\begin{array}{l}\text { System of } \\
\text { pumps and } \\
\text { piping to move } \\
\text { water from one } \\
\text { location to } \\
\text { another for } \\
\text { collection or } \\
\text { storage. }^{c}\end{array}$ & $\begin{array}{l}\text { Sewers can be } \\
\text { plugged to isolate } \\
\text { contaminated } \\
\text { waters in a specific }^{\text {location. }}{ }^{\mathrm{d}}\end{array}$ \\
\hline & & ADVANTAGES & $\begin{array}{l}\text { Large quantities } \\
\text { available COTS, } \\
\text { flexible material for } \\
\text { custom applications, } \\
\text { DIY }\end{array}$ & $\begin{array}{l}\text { COTS (plugs) or } \\
\text { existing } \\
\text { infrastructure } \\
\text { (diverters), DIY. } \\
\text { Ad hoc materials } \\
\text { (wood, polymer } \\
\text { sheeting) likely } \\
\text { available. }\end{array}$ & $\begin{array}{l}\text { Large quantitites } \\
\text { available COTS, } \\
\text { DIY. }\end{array}$ & $\begin{array}{l}\text { Several suppliers } \\
\text { (e.g., HESCO, } \\
\text { Hydro-Response, } \\
\text { Lester Solutions, } \\
\text { BigBags USA), } \\
\text { rapid setup. } \\
\text { Designed for } \\
\text { emergency } \\
\text { deployment. Proven } \\
\text { for flood control. }\end{array}$ & Rapid deployment. & $\begin{array}{l}\text { Large quantities } \\
\text { available COTS, } \\
\text { individual control } \\
\text { of piping. Isolates } \\
\text { water. }\end{array}$ & $\begin{array}{l}\text { Large quantities } \\
\text { available } \\
\text { COTS, various } \\
\text { sizes of pumps } \\
\text { and piping. }\end{array}$ & $\begin{array}{l}\text { Could potentially } \\
\text { isolate water for } \\
\text { subsequent } \\
\text { treatment or } \\
\text { transport. }\end{array}$ \\
\hline & & LIMITATIONS & $\begin{array}{l}\text { For shallow waters, } \\
\text { requires flat surface to } \\
\text { limit water intrusion. }\end{array}$ & $\begin{array}{l}\text { Large number of } \\
\text { intakes or } \\
\text { penetrations may } \\
\text { require custom } \\
\text { covers. }\end{array}$ & $\begin{array}{l}\text { Labor intensive. } \\
\text { Need to haul } \\
\text { necessary fill } \\
\text { material. }\end{array}$ & $\begin{array}{l}\text { Need to haul } \\
\text { necessary fill } \\
\text { material. }\end{array}$ & Availability. & $\begin{array}{l}\text { Knowledge of } \\
\text { piping system for } \\
\text { effective water } \\
\text { diversion. }\end{array}$ & $\begin{array}{l}\text { Availability of } \\
\text { larger pumps. }\end{array}$ & $\begin{array}{l}\text { Unsure of extent of } \\
\text { capability (trunk } \\
\text { lines, mains, sub- } \\
\text { main, branches). }\end{array}$ \\
\hline & & R\&D NEEDS & $\begin{array}{l}\text { Best practice } \\
\text { guidance (LR). }\end{array}$ & $\begin{array}{l}\text { Best practice } \\
\text { guidance (LR). }\end{array}$ & $\begin{array}{l}\text { Guidance on use } \\
\text { (LR). }\end{array}$ & $\begin{array}{l}\text { Guidance on use } \\
\text { (LR). }\end{array}$ & $\begin{array}{l}\text { Survey of inventory } \\
\text { (LR). Guidance on } \\
\text { use (LR). }\end{array}$ & $\begin{array}{l}\text { Strategy for use } \\
\text { (LR). Existence of } \\
\text { sewer and } \\
\text { topography maps } \\
\text { (LR). }\end{array}$ & $\begin{array}{l}\text { Guidance on } \\
\text { use (LR). } \\
\text { Survey of } \\
\text { inventory (LR). }\end{array}$ & $\begin{array}{l}\text { Survey of sewer } \\
\text { network mapping } \\
\text { and capabilities } \\
\text { (LR). }\end{array}$ \\
\hline
\end{tabular}

All images are personal photographs or courtesy of Shutterstock, except: (a) permission granted from Ultratech], (b) permission granted from Detroit Tarp, (c) New York Mass Transit Authority Creative Commons, (d) permission granted from Environmental Protection Agency. 
TABLE 3-5-2 Support Goal: Containment of Wastewater and Other Wastes for Scenario 2

\begin{tabular}{|c|c|c|c|c|c|c|c|c|c|c|c|c|}
\hline \multirow{2}{*}{ Scenario Description } & \multirow{2}{*}{ Summary } & \multirow{2}{*}{ Category } & \multicolumn{10}{|c|}{ Column Number } \\
\hline & & & 1 & 2 & 3 & 4 & $=$ & 6 & 7 & 8 & 9 & 10 \\
\hline \multirow{5}{*}{$\begin{array}{l}\text { Scenario 2: } \\
\text { What types of barriers } \\
\text { (for example, } \\
\text { sandbags and berms) } \\
\text { are available to collect } \\
\text { water generated during } \\
\text { its large-scale use } \\
\text { (millions of gallons) } \\
\text { over a large urban } \\
\text { footprint (for example, } \\
10 \text { square city blocks)? }\end{array}$} & \multirow{5}{*}{$\begin{array}{l}\text { - Inevitable leakage of } \\
\text { some waters is a } \\
\text { common problem for } \\
\text { these technologies. } \\
\text { - Many of the items } \\
\text { listed here are largely } \\
\text { available as COTS. } \\
\text { - Knowledge of the } \\
\text { topography of the } \\
\text { terrain will be } \\
\text { essential to contain } \\
\text { large volumes of } \\
\text { water. } \\
\text { - Guidance on their } \\
\text { specific use is lacking. } \\
\text { - Whether all major } \\
\text { cities have GIS sewer } \\
\text { maps is unknown. } \\
\text { - Knowledge of } \\
\text { whether sewer lines } \\
\text { can be plugged to hold } \\
\text { water in an individual } \\
\text { neighborhood or block } \\
\text { is lacking. }\end{array}$} & EQUIPMENT & $\begin{array}{l}\begin{array}{l}\text { Spill } \\
\text { barriers/bunds/berms. }\end{array} \\
\end{array}$ & $\begin{array}{l}\text { Drain covers or } \\
\text { diverters. }\end{array}$ & Sandbags. & $\begin{array}{l}\text { Flood control } \\
\text { barriers. }\end{array}$ & $\begin{array}{l}\text { Inflatable tube } \\
\text { barriers. }\end{array}$ & $\begin{array}{l}\text { Inflatable pipe } \\
\text { and sewer plugs. }\end{array}$ & $\begin{array}{l}\text { Pumps and } \\
\text { piping. }\end{array}$ & Flood barriers. & Sewer lines. & $\begin{array}{l}\text { Jersey } \\
\text { barriers. }\end{array}$ \\
\hline & & DESCRIPTION & \begin{tabular}{|l} 
Flexible temporary \\
berms to protect \\
drains, building \\
entryways, and \\
equipment from \\
shallow water.
\end{tabular} & $\begin{array}{l}\text { Specialty or ad } \\
\text { hoc covers can } \\
\text { protect intakes or } \\
\text { penetrations from } \\
\text { contaminated } \\
\text { water. }\end{array}$ & $\begin{array}{l}\text { Effective flood } \\
\text { control achieved } \\
\text { by building a wall } \\
\text { of stacked bags. }\end{array}$ & $\begin{array}{l}\text { Emergency barriers } \\
\text { supplied worldwide } \\
\text { during emergencies } \\
\text { to control and } \\
\text { contain water. }\end{array}$ & $\begin{array}{l}\text { Inflatable PVC } \\
\text { tubes to } \\
\text { contain/divert } \\
\text { water. }\end{array}$ & $\begin{array}{l}\text { Inflatable plugs } \\
\text { for plugging } \\
\text { sewer lines. }\end{array}$ & $\begin{array}{l}\text { System of } \\
\text { pumps and } \\
\text { piping to move } \\
\text { water from one } \\
\text { location to } \\
\text { another for } \\
\text { collection or } \\
\text { storage. }\end{array}$ & $\begin{array}{l}\text { Can be used to } \\
\text { limit water } \\
\text { intrusion. } \\
\end{array}$ & $\begin{array}{l}\text { Sewers can be } \\
\text { plugged to } \\
\text { isolate } \\
\text { contaminated } \\
\text { waters in a } \\
\text { specific } \\
\text { location. }^{\text {d }}\end{array}$ & $\begin{array}{l}\text { Create a } \\
\text { temporary } \\
\text { dike with } \\
\text { Jersey } \\
\text { barriers or } \\
\text { lined k-rails } \\
\text { to divert } \\
\text { water to a } \\
\text { central } \\
\text { location. }\end{array}$ \\
\hline & & ADVANTAGES & $\begin{array}{l}\text { Large quantities } \\
\text { available COTS, } \\
\text { flexible material for } \\
\text { custom applications, } \\
\text { DIY. }\end{array}$ & $\begin{array}{l}\text { COTS (plugs) or } \\
\text { existing } \\
\text { infrastructure } \\
\text { (diverters). Ad } \\
\text { hoc materials } \\
\text { (wood, polymer } \\
\text { sheeting) likely } \\
\text { available. }\end{array}$ & $\begin{array}{l}\text { Large quantities } \\
\text { available COTS, } \\
\text { DIY. }\end{array}$ & $\begin{array}{l}\text { Several suppliers } \\
\text { (e.g., HESCO, } \\
\text { Hydro-Response, } \\
\text { Lester Solutions,, } \\
\text { BigBags USA), } \\
\text { rapid setup. } \\
\text { Designed for } \\
\text { emergency } \\
\text { deployment. } \\
\text { Proven for flood } \\
\text { control. }\end{array}$ & $\begin{array}{l}\text { Rapid } \\
\text { deployment. }\end{array}$ & $\begin{array}{l}\text { Large quantities } \\
\text { available COTS, } \\
\text { individual } \\
\text { control of piping, } \\
\text { isolates water. }\end{array}$ & $\begin{array}{l}\text { Large quantities } \\
\text { available } \\
\text { COTS, various } \\
\text { sizes of pumps } \\
\text { and piping. }\end{array}$ & $\begin{array}{l}\text { Plastic portable } \\
\text { flood barrier. }\end{array}$ & $\begin{array}{l}\text { Could potentially } \\
\text { isolate water for } \\
\text { subsequent } \\
\text { treatment or } \\
\text { transport. }\end{array}$ & $\begin{array}{l}\text { Large } \\
\text { quantities } \\
\text { available } \\
\text { COTS, ad hoc } \\
\text { construction. }\end{array}$ \\
\hline & & LIMITATIONS & $\begin{array}{l}\text { For shallow waters, } \\
\text { requires flat surface } \\
\text { to limit water } \\
\text { intrusion. }\end{array}$ & $\begin{array}{l}\text { Large number of } \\
\text { intakes or } \\
\text { penetrations may } \\
\text { require custom } \\
\text { covers. }\end{array}$ & $\begin{array}{l}\text { Labor intensive. } \\
\text { Need to haul } \\
\text { necessary fill } \\
\text { material. }\end{array}$ & $\begin{array}{l}\text { Need to haul } \\
\text { necessary fill } \\
\text { material. }\end{array}$ & Availability. & $\begin{array}{l}\text { Knowledge of } \\
\text { piping system for } \\
\text { effective water } \\
\text { diversion. }\end{array}$ & $\begin{array}{l}\text { Availability of } \\
\text { larger pumps. }\end{array}$ & $\begin{array}{l}\text { Availability, for } \\
\text { shallow waters. }\end{array}$ & $\begin{array}{l}\text { Unsure of extent } \\
\text { of capability } \\
\text { (trunk lines, } \\
\text { mains, sub- } \\
\text { mains, branches) }\end{array}$ & $\begin{array}{l}\text { For shallow } \\
\text { waters. }\end{array}$ \\
\hline & & R\&D NEEDS & $\begin{array}{l}\text { Best practice } \\
\text { guidance (LR). }\end{array}$ & $\begin{array}{l}\text { Best practice } \\
\text { guidance (LR). }\end{array}$ & $\begin{array}{l}\text { Guidance on use } \\
\text { (LR). }\end{array}$ & $\begin{array}{l}\text { Guidance on use } \\
\text { (LR). }\end{array}$ & $\begin{array}{l}\text { Survey of } \\
\text { inventory (LR). } \\
\text { Guidance on use } \\
\text { (LR). }\end{array}$ & $\begin{array}{l}\text { Strategy for use } \\
\text { (LR). Existence } \\
\text { of sewer and } \\
\text { topography maps } \\
\text { (LR). }\end{array}$ & $\begin{array}{l}\text { Guidance on } \\
\text { use (LR). } \\
\text { Survey of } \\
\text { inventory (LR). }\end{array}$ & $\begin{array}{l}\text { Survey of } \\
\text { inventory (LR). } \\
\text { Guidance on use } \\
\text { (LR). }\end{array}$ & $\begin{array}{l}\text { Strategy for use } \\
\text { (LR). Survey of } \\
\text { sewer network } \\
\text { mapping and } \\
\text { capabilities (LR). }\end{array}$ & $\begin{array}{l}\text { Guidance of } \\
\text { use (LR). }\end{array}$ \\
\hline
\end{tabular}

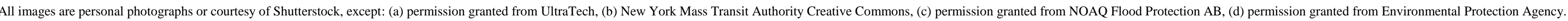


TABLE 3-5-3 Support Goal: Containment of Wastewater and Other Wastes for Scenario 3

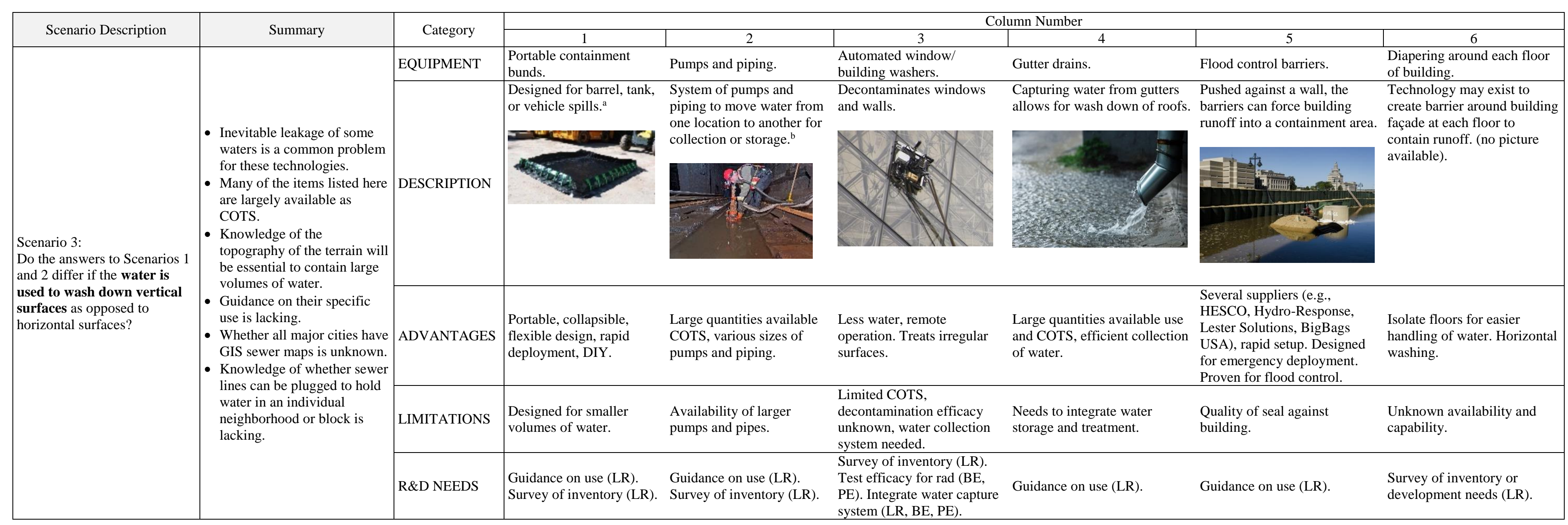

All images are personal photographs or courtesy of Shutterstock, except: (a) permission granted from Ultratech and (b) New York Mass Transit Authority Creative Commons. 
TABLE 3-5-4 Support Goal: Containment of Wastewater and Other Wastes for Scenario 4

\begin{tabular}{|c|c|c|c|c|c|c|c|c|c|c|c|}
\hline \multirow{2}{*}{ Scenario Description } & \multirow{2}{*}{ Summary } & \multirow{2}{*}{ Category } & \multicolumn{9}{|c|}{ Column Number } \\
\hline & & & 1 & 2 & 3 & 4 & 5 & 6 & 7 & 8 & 9 \\
\hline \multirow{6}{*}{$\begin{array}{l}\text { Scenario 4: } \\
\text { If the water is collected } \\
\text { at the point of use, } \\
\text { what containers, } \\
\text { vessels, or facilities are } \\
\text { available to store the } \\
\text { water generated in } \\
\text { Scenarios } 1 \text { through } 3 \\
\text { until it can be processed } \\
\text { or transported? }\end{array}$} & \multirow{6}{*}{$\begin{array}{l}\text { - Common unresolved } \\
\text { issues: (1) Availability of } \\
\text { privately-owned } \\
\text { equipment. It may already } \\
\text { be in commercial or } \\
\text { private use, or owners } \\
\text { may be reluctant to allow } \\
\text { its use in a radioactive } \\
\text { environment without prior } \\
\text { agreements in place. (2) } \\
\text { Ability to decontaminate } \\
\text { equipment afterward for } \\
\text { unrestricted use. (3) Need } \\
\text { for a sufficient available } \\
\text { footprint to accommodate } \\
\text { storage unit and need for } \\
\text { approval for its siting, } \\
\text { construction, and } \\
\text { operation. } \\
\text { - Many of the water storage } \\
\text { options presented here are } \\
\text { explained in more detail in } \\
\text { EPA 817-B-12-002. }\end{array}$} & EQUIPMENT & Large tanks. & $\begin{array}{l}\text { Intermediate bulk } \\
\text { containers (IBCs). }\end{array}$ & Road tankers. & Baker trucks. & $\begin{array}{l}\text { Locally } \\
\text { constructed, lined } \\
\text { lagoons or pools. }\end{array}$ & Pumps and piping. & $\begin{array}{l}\text { Inflatable pipe and } \\
\text { sewer plugs. }\end{array}$ & $\begin{array}{l}\text { Fuel/water } \\
\text { bladders. }\end{array}$ & Sewer lines. \\
\hline & & & $\begin{array}{l}\text { Any number of } \\
\text { tank sizes available } \\
\text { or in use for liquid } \\
\text { storage. }\end{array}$ & $\begin{array}{l}\text { Medium-sized } \\
\text { container for liquids. }\end{array}$ & $\begin{array}{l}\text { Allow for immediate } \\
\text { transport of water } \\
\text { following filling. }\end{array}$ & $\begin{array}{l}\text { Can be used to store } \\
\text { or treat water as an } \\
\text { ad hoc filter bed. } \\
\text { https://www.bakerco }\end{array}$ & $\begin{array}{l}\text { Local structure for } \\
\text { containing water. }\end{array}$ & $\begin{array}{l}\text { System of pumps } \\
\text { and piping to move } \\
\text { water from one } \\
\text { location to another }\end{array}$ & $\begin{array}{l}\text { Using sewer flow } \\
\text { maps, plug the } \\
\text { sewer lines to } \\
\text { force water to a }\end{array}$ & $\begin{array}{l}\text { Flexible bladders } \\
\text { for holding water } \\
\text { or fuel. }\end{array}$ & $\begin{array}{l}\text { Sewers can be } \\
\text { plugged to isolate } \\
\text { contaminated } \\
\text { waters in a specific }\end{array}$ \\
\hline & & DESCRIPTION & $\stackrel{x=0}{\longrightarrow}$ & & & & & 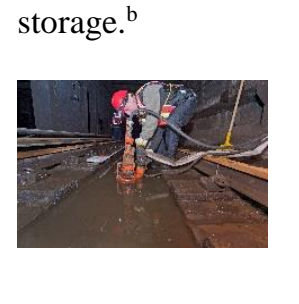 & $\begin{array}{l}\text { collection and } \\
\text { treatment. }\end{array}$ & & \\
\hline & & ADVANTAGES & $\begin{array}{l}\text { Huge capacity, } \\
\text { Fukushima } \\
\text { experience. }\end{array}$ & $\begin{array}{l}\text { Mass produced, } \\
\text { easily transported, } \\
\text { easily stacked. } \\
\text { Compatible with } \\
\text { pumps and mixers, } \\
\text { Fukushima } \\
\text { experience. }\end{array}$ & $\begin{array}{l}\text { Large quantities } \\
\text { available, network, } \\
\text { existing operators, } \\
\text { large volume (more } \\
\text { than } \\
10,000 \text { gallons). }\end{array}$ & $\begin{array}{l}\text { Commonly used, } \\
\text { large capacity. }\end{array}$ & $\begin{array}{l}\text { Huge capacity, } \\
\text { local, versatile } \\
\text { design, prior } \\
\text { knowledge in } \\
\text { chemical industry. }\end{array}$ & $\begin{array}{l}\text { Large quantities } \\
\text { available COTS, } \\
\text { various sizes of } \\
\text { pumps and piping. }\end{array}$ & $\begin{array}{l}\text { Large quantities } \\
\text { available COTS, } \\
\text { individual control } \\
\text { of piping, isolates } \\
\text { water. }\end{array}$ & $\begin{array}{l}\text { Large capacity } \\
\text { (more than } \\
\text { 200,000 gallons), } \\
\text { rapid deployment. }\end{array}$ & $\begin{array}{l}\text { Potentially isolate } \\
\text { water for } \\
\text { subsequent } \\
\text { treatment or } \\
\text { transport. }\end{array}$ \\
\hline & & LIMITATIONS & Slow construction. & $\begin{array}{l}\text { Smaller volume (up } \\
\text { to } \sim 550 \text { gallons). }\end{array}$ & $\begin{array}{l}\text { Approved routes, } \\
\text { availability. }\end{array}$ & Availability. & $\begin{array}{l}\text { Siting. Attracts } \\
\text { wildlife. }\end{array}$ & $\begin{array}{l}\text { Availability of } \\
\text { larger pumps and } \\
\text { pipes. }\end{array}$ & $\begin{array}{l}\text { Knowledge of } \\
\text { piping system for } \\
\text { effective water } \\
\text { diversion. }\end{array}$ & Availability. & $\begin{array}{l}\text { Unsure of extent of } \\
\text { capability (trunk } \\
\text { lines, mains, sub- } \\
\text { main, branches). }\end{array}$ \\
\hline & & R\&D NEEDS & $\begin{array}{l}\text { Guidance for use } \\
\text { (LR). }\end{array}$ & $\begin{array}{l}\text { Method to monitor } \\
\text { each bin or bins } \\
\text { (LR, BE, PE). } \\
\text { Guidance on use } \\
\text { (LR). }\end{array}$ & $\begin{array}{l}\text { Clarify transport } \\
\text { regulations (LR). } \\
\text { Decontamination } \\
\text { guidance (LR). }\end{array}$ & $\begin{array}{l}\text { Survey of inventory } \\
\text { (LR). Clarify } \\
\text { transport regulations } \\
\text { (LR). } \\
\text { Decontamination } \\
\text { guidance (LR). } \\
\text { Guidance for use as } \\
\text { filter bed (LR, BE, } \\
\text { PE). }\end{array}$ & $\begin{array}{l}\text { Design guidance } \\
\text { (LR). }\end{array}$ & $\begin{array}{l}\text { Guidance on use } \\
\text { (LR). Survey of } \\
\text { inventory (LR). }\end{array}$ & $\begin{array}{l}\text { Strategy for use } \\
\text { (LR). Existence of } \\
\text { sewer and } \\
\text { topography maps } \\
\text { (LR). }\end{array}$ & $\begin{array}{l}\text { Survey of } \\
\text { inventory (LR). }\end{array}$ & $\begin{array}{l}\text { Survey of sewer } \\
\text { network mapping } \\
\text { and capabilities } \\
\text { (LR). }\end{array}$ \\
\hline
\end{tabular}

All images are personal photographs or courtesy of Shutterstock, except: (a) permission granted from ModuTank, (b) New York Mass Transit Authority Creative Commons, and (c) permission granted from Environmental Protection Agency. 
TABLE 3-5-5 Support Goal: Containment of Wastewater and Other Wastes for Scenario 5

\begin{tabular}{|c|c|c|c|c|c|c|c|c|c|c|c|}
\hline \multirow{2}{*}{$\begin{array}{c}\text { Scenario } \\
\text { Description }\end{array}$} & \multirow{2}{*}{ Summary } & \multirow{2}{*}{ Category } & \multicolumn{9}{|c|}{ Column Number } \\
\hline & & & 1 & 2 & 3 & 4 & 5 & 6 & 7 & 8 & 9 \\
\hline \multirow{5}{*}{$\begin{array}{l}\text { Scenario 5: } \\
\text { If the water } \\
\text { penetrates the } \\
\text { sewer system } \\
\text { and can be } \\
\text { diverted at a } \\
\text { downstream } \\
\text { collection site } \\
\text { (e.g., just prior } \\
\text { to entering the } \\
\text { water } \\
\text { reclamation } \\
\text { district or } \\
\text { wastewater } \\
\text { treatment } \\
\text { plant), what } \\
\text { containers, } \\
\text { vessels, or } \\
\text { facilities are } \\
\text { available to } \\
\text { store the water } \\
\text { until it can be } \\
\text { processed or } \\
\text { transported? }\end{array}$} & \multirow{5}{*}{$\begin{array}{l}\text { - Common } \\
\text { unresolved } \\
\text { issues: (1) } \\
\text { Availability of } \\
\text { privately- } \\
\text { owned } \\
\text { equipment. It } \\
\text { may already be } \\
\text { in commercial } \\
\text { or private use, } \\
\text { or owners may } \\
\text { be reluctant to } \\
\text { allow its use in } \\
\text { a radioactive } \\
\text { environment } \\
\text { without prior } \\
\text { agreements in } \\
\text { place. (2) } \\
\text { Ability to } \\
\text { decontaminate } \\
\text { equipment } \\
\text { afterward for } \\
\text { unrestricted } \\
\text { use. (3) Need } \\
\text { for an approved } \\
\text { storage } \\
\text { footprint to } \\
\text { accommodate } \\
\text { storage unit and } \\
\text { need for } \\
\text { approval for its } \\
\text { siting, } \\
\text { construction, } \\
\text { and oneration }\end{array}$} & EQUIPMENT & Bund liners. & Fuel/water bladders. & Dracone barge. & Huge tanks. & $\begin{array}{l}\text { Locally constructed, } \\
\text { lined lagoons or } \\
\text { pools. }\end{array}$ & Pumps and piping. & Frack water tanks. & Baker trucks. & Railcars. \\
\hline & & DESCRIPTION & $\begin{array}{l}\text { Designed to store } \\
\text { water until it has } \\
\text { been treated or } \\
\text { evaporates. }\end{array}$ & $\begin{array}{l}\text { Flexible bladders to } \\
\text { hold water. }\end{array}$ & $\begin{array}{l}\text { United Kingdom } \\
\text { (UK) product to } \\
\text { transport liquids or } \\
\text { contain oil spills } \\
\text { and tow it away. } \\
\text { http://www.trellebo } \\
\text { rg.com/en/orr-- } \\
\text { solutions/flexible-- } \\
\text { containment-- } \\
\text { solutions }\end{array}$ & $\begin{array}{l}\text { Used to store liquids } \\
\text { for distribution or } \\
\text { treatment. }\end{array}$ & $\begin{array}{l}\text { Local structure for } \\
\text { containing water. }\end{array}$ & $\begin{array}{l}\text { System of pumps and } \\
\text { piping to move water } \\
\text { from one location to } \\
\text { another for collection or } \\
\text { storage. }\end{array}$ & $\begin{array}{l}\text { Designed to hold } \\
\text { water onsite until } \\
\text { treatment. }\end{array}$ & $\begin{array}{l}\text { Can be used to store or } \\
\text { treat water as an ad hoc } \\
\text { filter bed. } \\
\text { https://www.bakercorp. } \\
\text { com/en-us/ }\end{array}$ & $\begin{array}{l}\text { Can transport } \\
\text { contaminated water. }\end{array}$ \\
\hline & & ADVANTAGES & $\begin{array}{l}\text { Liners for a pit, } \\
\text { natural evaporation. }\end{array}$ & $\begin{array}{l}\text { Large capacity } \\
\text { (more than } \\
200,000 \text { gallons), } \\
\text { rapid deployment. }\end{array}$ & $\begin{array}{l}\text { Highly portable } \\
\text { when empty. } \\
\text { Deployed for } \\
\text { emergency spills. } \\
\text { Huge capacity, } \\
\text { rugged. }\end{array}$ & Enormous capacity. & $\begin{array}{l}\text { Huge capacity, } \\
\text { local, versatile } \\
\text { design, prior } \\
\text { experience in } \\
\text { chemical industry. }\end{array}$ & $\begin{array}{l}\text { Large quantities } \\
\text { available COTS, } \\
\text { various sizes of pumps } \\
\text { and piping. }\end{array}$ & $\begin{array}{l}\text { Huge capacity, } \\
\text { evaporation. }\end{array}$ & $\begin{array}{l}\text { Commonly used, large } \\
\text { capacity. }\end{array}$ & $\begin{array}{l}\text { Large network and } \\
\text { variety of rolling stock } \\
\text { for hauling, hazardous } \\
\text { transport ongoing. }\end{array}$ \\
\hline & & LIMITATIONS & $\begin{array}{l}\text { Siting, availability. } \\
\text { Attracts wildlife. }\end{array}$ & Availability. & Availability. & Availability. & $\begin{array}{l}\text { Siting. Attracts } \\
\text { wildlife. }\end{array}$ & $\begin{array}{l}\text { Availability of larger } \\
\text { pumps. }\end{array}$ & $\begin{array}{l}\text { Siting, availability. } \\
\text { Attracts wildlife. }\end{array}$ & $\begin{array}{l}\text { Approved routes, } \\
\text { availability. }\end{array}$ & $\begin{array}{l}\text { Agreements for use, } \\
\text { no predetermined } \\
\text { destination, } \\
\text { availability of tanker } \\
\text { cars. }\end{array}$ \\
\hline & & R\&D NEEDS & $\begin{array}{l}\text { Survey of inventory } \\
\text { (LR). }\end{array}$ & $\begin{array}{l}\text { Survey of inventory } \\
\text { (LR). }\end{array}$ & $\begin{array}{l}\text { Survey of } \\
\text { inventory (LR). }\end{array}$ & $\begin{array}{l}\text { Survey of inventory } \\
\text { (LR). }\end{array}$ & $\begin{array}{l}\text { Design guidance } \\
\text { (LR). }\end{array}$ & $\begin{array}{l}\text { Guidance on use (LR). } \\
\text { Survey of inventory } \\
\text { (LR). }\end{array}$ & $\begin{array}{l}\text { Survey of inventory } \\
\text { (LR). }\end{array}$ & $\begin{array}{l}\text { Survey of inventory } \\
\text { (LR). Clarify transport } \\
\text { regulations (LR). } \\
\text { Decontamination } \\
\text { guidance (LR). } \\
\text { Guidance for use as } \\
\text { filter bed (LR, BE, PE). }\end{array}$ & $\begin{array}{l}\text { Survey of inventory } \\
\text { (LR). Clarify transport } \\
\text { regulations (LR). } \\
\text { Decontamination } \\
\text { guidance (LR). }\end{array}$ \\
\hline
\end{tabular}

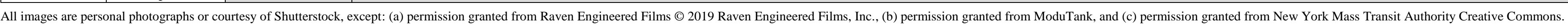


TABLE 3-5-6 Support Goal: Containment of Wastewater and Other Wastes for Scenario 6

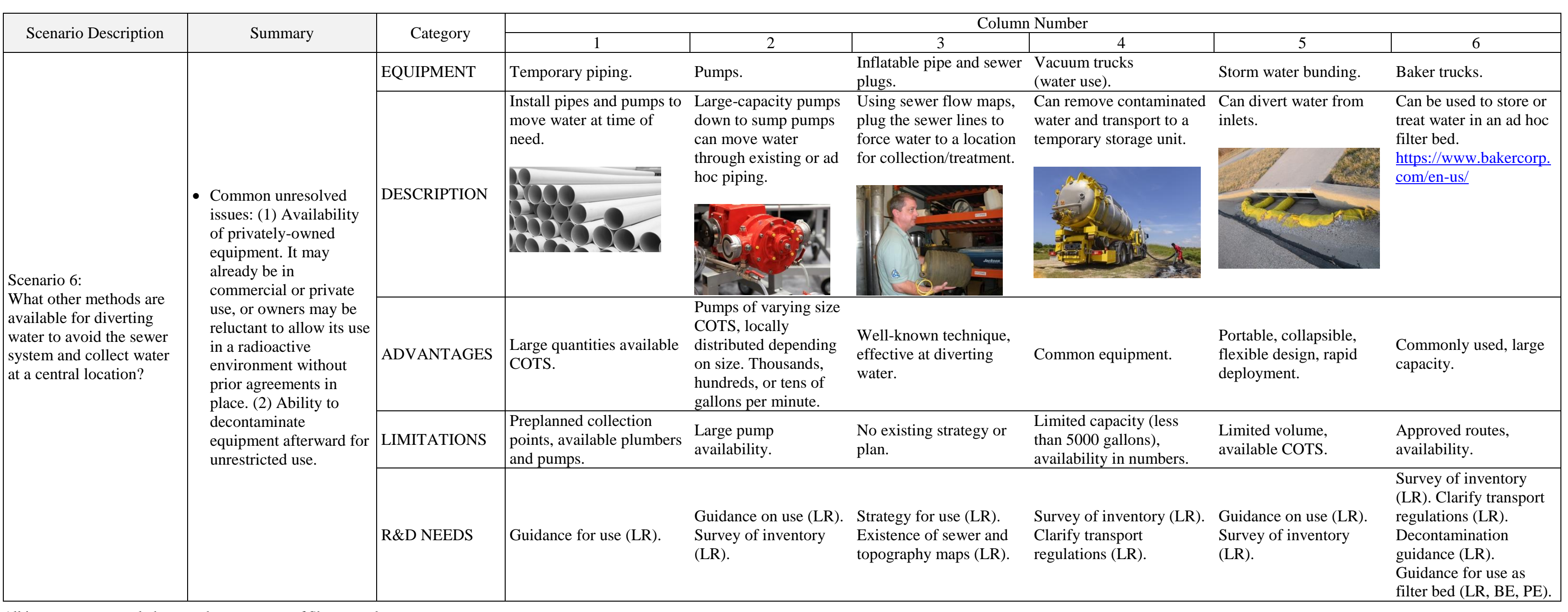


TABLE 3-5-7 Support Goal: Containment of Wastewater and Other Wastes for Scenarios 7 and 8

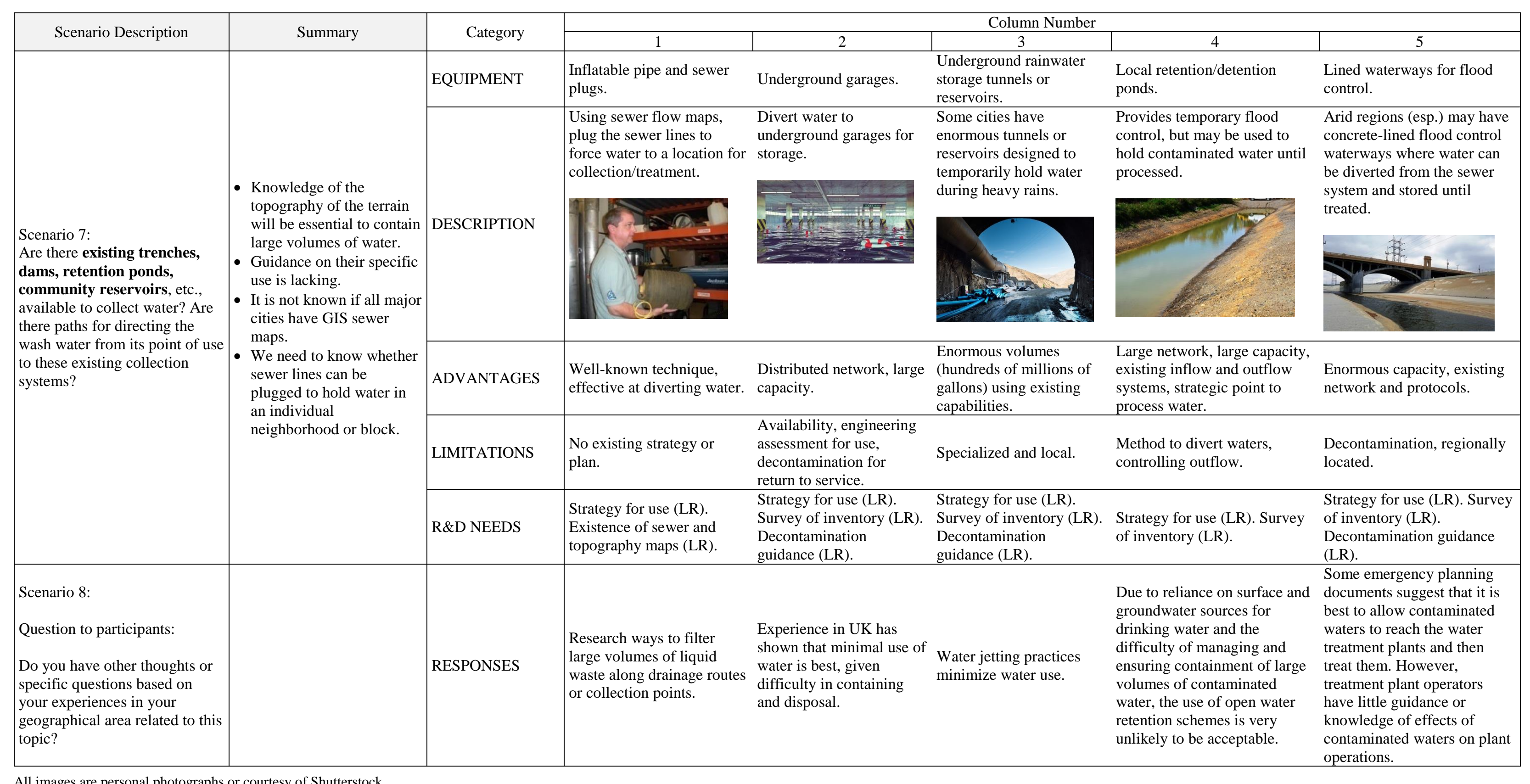


TABLE 3-5-8 Support Goal Training: Containment of Wastewater and Other Wastes for Scenarios 1-8

\begin{tabular}{|c|c|c|}
\hline Support Goal Training: Scenarios 1-8 & Summary & Responses \\
\hline $\begin{array}{l}\text { Question to participants: } \\
\text { What are your thoughts and } \\
\text { recommendations on availability of } \\
\text { trained human assets and training of } \\
\text { additional assets that will likely be } \\
\text { needed in order to accomplish the } \\
\text { scenarios under this goal? (Training } \\
\text { will be a significant effort and an } \\
\text { additional limiting factor in any } \\
\text { response. It may need to be addressed } \\
\text { more thoroughly in the future, but input } \\
\text { is needed to help guide how training } \\
\text { guidance should be developed.) }\end{array}$ & $\begin{array}{l}\text { Wide-area radiological } \\
\text { contamination incidents are rare, } \\
\text { and a response to such an incident } \\
\text { will require tremendous human } \\
\text { assets. Lessons learned from the } \\
\text { cleanup efforts in Japan showed } \\
\text { that many thousands of individuals } \\
\text { each day are engaged in clean-up } \\
\text { activities. }\end{array}$ & $\begin{array}{l}\text { Large-scale wastewater management would require } \\
\text { specialist with floodwater management expertise } \\
\text { supported by health physics monitors and } \\
\text { decommissioning specialists. }\end{array}$ \\
\hline
\end{tabular}




\section{RANKING THE EQUIPMENT}

Using Tables 3-1-1 through 3-5-8, we gathered responses from the various SMEs to rank the equipment. To do so, participants were asked to consider the listed R\&D needs and determine what equipment they would prioritize based on likely use and need for additional knowledge of its use, function, or efficacy. They ranked these items according to what would provide the most benefit from additional R\&D (rank 1) to less benefit (rank 3). Importantly, the results of the rankings may be used to prioritize future work, including best practices, operational guides, and work to adapt the equipment for radioactive contamination scenarios. Organized by Support Goal, these were the technologies ranked highest by the SMEs.

Survey and Monitoring

- Small vehicles with commercial gamma detector.

- Smart phones as radiation detectors.

- Drones equipped with gamma cameras.

- USB-type dosimeters.

- Air filters from home and vehicles for monitoring resuspended contamination.

- Radiation monitors on taxis, buses, ambulances, delivery vehicles, etc., with GPS tracking.

- Speedbump detectors to monitor vehicles.

- Portal or other stationary monitors for vehicles.

Mitigation of Received Dose to First Responders

- Street sweeper (retrofitted) with on-board detectors and GPS tracking.

- Portable water trailer to wash street.

- Soil stabilizer spray systems to control airborne dust.

- Chip sealer (emulsion) distributor to cover roads and reduce resuspension hazard.

- Intermediate bulk container with polymer (paint) sprayer to cover streets.

- Mobile spray unit to cover surfaces with films.

Support Goal: Decontamination (gross and final)

- Salt spreaders to improve decontamination.

- Pressure washer systems for decontamination.

- Mobile water filtration systems for captured waters.

- Wash units used for freight and passenger trains.

- Best practices for cleaning hospitals and commercial nuclear power plants.

- Automated window/building washers.

Waste Management

- Distributed waste skippers/freight containers for contaminated trash.

- 1-ton solid waste bags for containment and transport from residences and businesses. 
- Tagging and tracking systems for waste bags and units.

- Procedures for qualifying transport of waste via freight container.

\section{Containment of Wastewater and Other Wastes}

- Drain covers and flood control barriers to divert and collect water.

- Lined lagoons for storage of contaminated water.

- Monitors for sewer lines.

- Pumps and ad hoc piping to transfer contaminated water from

- sewer lines to container trucks or rail tankers

- reservoir to rail tankers. 


\section{EXISTING GUIDANCE}

Table 5-1 lists some existing documents and information that cover topics relevant to this report.

TABLE 5-1 Existing Guidance

\begin{tabular}{|c|c|}
\hline Topic & Resource \\
\hline $\begin{array}{l}\text { Technical } \\
\text { Documents }\end{array}$ & 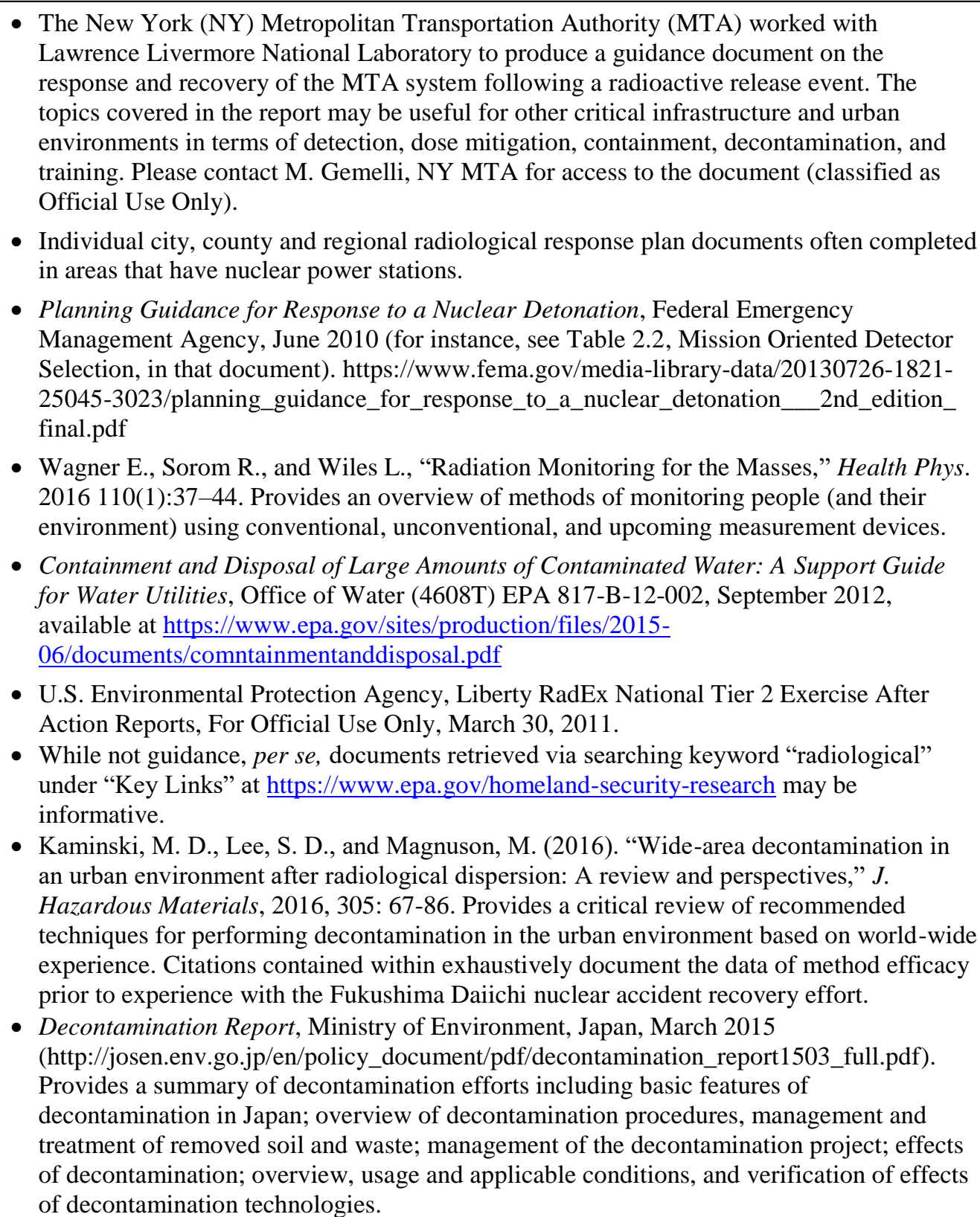 \\
\hline
\end{tabular}


TABLE 5-1 (Cont.)

\begin{tabular}{|c|c|}
\hline Topic & Resource \\
\hline $\begin{array}{l}\text { Social Media } \\
\text { and Apps }\end{array}$ & $\begin{array}{l}\text { - Applications such as Zello may be used to communicate effectively to expedite and } \\
\text { coordinate the recovery effort, especially self-help efforts. Zello is an application that } \\
\text { emulates "push-to-talk (PTT) walkie-talkies over cell phone networks. The apps are } \\
\text { available for Android, iOS, Blackberry, Windows Phone, Windows PC, rugged mobile } \\
\text { devices and two-way radios." } \\
\text { - The volunteer organization Cajun Navy_-founded in } 2005 \text { following Hurricane Katrina- } \\
\text { is mobilizing rescuers through a channel called "Texas search and rescue." The PTT app } \\
\text { lets users send voice messages to different channels. Anyone listening to the channel can } \\
\text { hear these messages. People can also talk to each other in private chats. } \\
\text { - We may want to research apps or social networking employed during Katrina, Sandy, and } \\
\text { Harvey that were used to recruit equipment for cleanup (e.g., dump trucks during Sandy) } \\
\text { or rescues (boats during Harvey). Other apps of interest may include FreeCast, Glide, } \\
\text { Mumble, and WhatsApp. } \\
\text { - Florida State University and the DOE in collaboration with other SMEs developed the } \\
\text { Deactivation \& Decommissioning Knowledge Management Information Tool (D\&D KM- } \\
\text { IT). This web-based tool's (https://www.dndkm.org/Default.aspx) objective is to "provide } \\
\text { single-point access into the collective knowledge-base of the D\&D community within and } \\
\text { outside of DOE." It provides searchable information on such areas as technology } \\
\text { description and use, best practices, lessons learned, and training related to decontamination } \\
\text { and decommissioning of facilities. } \\
\text { - The Rad Decon tool found in the RadResponder app } \\
\text { (https://www.radresponder.net/\#home) helps identify methods for decontamination based } \\
\text { on the data primarily found in the UK Recovery Handbooks } \\
\text { (https://www.gov.uk/government/publications/uk-recovery-handbooks-for-radiation- } \\
\text { incidents-2015). }\end{array}$ \\
\hline $\begin{array}{l}\text { Developing } \\
\text { Programs }\end{array}$ & $\begin{array}{l}\text { The Defense Advanced Research Projects Agency, Defense Science Office (DARPA-DSO) } \\
\text { sponsors a program called SIGMA, "Cost-effective, operationally practical, continuous city- } \\
\text { scale nuclear and radiological WMD detection capability." 10,000 personal radiation } \\
\text { detectors and more than } 200 \text { large detectors (vehicle mounted) provide continuous } \\
\text { measurements of a city. Scheduled to operationalize wide-area monitoring capability in } 2017 \\
\text { (Anne Fischer and Mark Wrobel, Program Managers). }\end{array}$ \\
\hline
\end{tabular}




\section{CRITICAL INFRASTRUCTURE EVALUATION}

The potential implementation of equipment for critical infrastructures was discussed throughout this project. This section summarizes the outcomes from these discussions and information gathering activities. Critical infrastructure is defined by DHS in terms of its relative importance to the stability of numerous sectors in the United States (https://www.dhs.gov/criticalinfrastructure-sectors, accessed April 2018): Chemical Sector, Commercial Facilities Sector, Communications Sector, Critical Manufacturing Sector, Dams Sector, Defense Industrial Base Sector, Emergency Services Sector, Energy Sector, Financial Services Sector, Food and Agriculture Sector, Government Facilities Sector, Healthcare and Public Health Sector, Information Technology Sector, Nuclear Reactors, Materials, and Waste Sector, Sector-Specific Agencies, Transportation Systems Sector, Water and Wastewater Systems Sector. The technologies described in the Section 3 tables have been primarily evaluated in the context of roadways, buildings, and open space areas found in the large urban environments of the United States. Within such, aspects of these 16 sectors are included where the needs include addressing affected paved areas, common building materials, vehicles, waterways, and green spaces. However, specific infrastructure may introduce new constraints and variables that have not been addressed. In addition, equipment and capabilities unique to the critical infrastructure may be used during the response and recovery phases to mitigate direct and indirect effects of a widearea radioactive contamination event. For completeness, a cursory description of specific infrastructure systems is presented below.

\subsection{COMMUTER RAIL}

Information regarding the public commuter rail infrastructure was presented by the Mass Transit Authority (MTA) of New York City. MTA provided a guidance document entitled "New York City Transit Grand Central Station Pre-Incident Radiological Dispersal Response and Recovery Plan" (LLNL-TM-64442, June 28, 2003). In it, a thorough evaluation of the commuter system is presented and a plan "designed to be an operationally focused, hands-on guide to help direct immediate actions to minimize the consequences of a radiological dispersal incident and to facilitate the preparation of a post-incident response and recovery plan and facility-specific strategies to support restoration of [Grand Central Station] service after a radiological dispersal incident." Decontamination strategies used the best techniques and information known to date.

\subsection{FREIGHT RAIL}

A Regional Environment Manager and Dangerous Goods/Emergency Response Preparedness officer from a Class I, intercontinental, freight rail carrier provided input into this study. Such a large rail carrier has extensive internal resources to ensure continuous operation of their fleet. Some of the techniques that are available in an emergency include: 
- Ability to retrofit rail cars and tunnels to add power washing systems for decontamination of engines and rolling stock or to use such systems to decontaminate an inventory of contaminated personal and commercial vehicles.

- Existing rail cars with vacuum units designed to remove debris from the right-of-way.

- Spill control systems for the rail system.

- 20,000-gallon portable tanks.

- Large open spaces along right-of-ways to store or set up unit operations.

- Ballast removal cars.

- Excavators mounted on rail cars.

- Power washing systems for heavily contaminated areas (designed specifically for hazardous cargo in mind).

- Expertise and equipment necessary to set up ad hoc piping systems.

- Expertise and equipment necessary to transport large volumes of liquids from rail cars to fixed storage vessels or from locations off-site into storage vessels on rail.

\subsection{WATER SUPPLY}

Meetings with experts at public water supply systems were not possible. However, basic information on the unit operations was gathered. Intake of water from fresh water sources such as lakes and rivers commonly incorporate a number of unit operations to ensure the safety of the water supply. These may include chemical treatment (e.g., with activated carbon polyphosphate, chlorine, fluoride, aluminum sulfate and polyelectrolyte), sedimentation, and filtration. Each of these steps accomplishes a different goal, such as eliminating odors, improving taste, killing bacteria, removing micro-organisms and suspended solids, or improving dental hygiene. Additional information about these and other steps can be found in EPA's "Drinking Water Treatability Database" (https://oaspub.epa.gov/tdb/pages/general/home.do).

As an example, the City of Chicago employs a process common to the industry (https://www.cityofchicago.org/city/en/depts/water/supp_info/education/water_treatment.html). The process consists of the following steps: water from Lake Michigan 1) enters the intake tunnel at depths of 20 to 30 feet, 2) passes to the purification plant through a tunnel beneath the lake bed, and 3) is filtered through eight traveling screens to catch coarse debris. Then, the water 4 ) is pumped up to the first chemical treatment and 5) flows through the chemical application channels. Afterward, 6) it flows through mixing basins where a flocculation process begins. This water 7) passes into settling basins to allow the floc to settle and 8) is filtered through graded sand and gravel beds to clarify the water. Then, the filtered water 9) flows into clear wells and is 
treated by another chemical application before the finished water 10) enters reservoirs and flows to the distribution system.

Several chemicals are used during the process: chlorine to disinfect the water; aluminum sulfate or an alum and polymer as flocculants; polyphosphates to protect pipes from leaching heavy metals such as lead; activated carbon to remove tastes and odors; and fluoride for dental hygiene. The amount of each added chemical is relatively small (approximately one teaspoon per 100 gallons or 15 parts of chemical to 1 million parts of water). ${ }^{4}$

In view of these types of unit operations, there appears to be opportunities to mitigate the effects of contamination to the water supply. Existing filtration beds may be modified to include selective sorbent material to help remove the contaminants prior to entering the downstream filtration systems. Also, contaminated particulate may be effectively eliminated with existing unit operations although the concentration of radionuclides inside the supply system will require consideration to avoid health hazards associated with elevated radiation fields.

\subsection{WASTE WATER TREATMENT PLANTS}

Meetings were held with members of the Water Environment Research Foundation (WERF, currently known as the Water Research Foundation), experts at the Metropolitan Water Reclamation District (MWRD) of Greater Chicago, and the Orange County Water District. In the meeting with WERF, it was clearly stated that the wastewater districts were not equipped to handle radioactively contaminated waters. Moreover, they were uncertain as to the effect such waters would have on unit operations, such as the health of the bacterial colonies, and were also unaware of studies predicting the fate of radioactive contaminants in the unit operations. They recommended that water be diverted as much as possible to avoid infiltration into the plant.

Afterward, meetings were held with members of MWRD to discuss Chicago plant operations and potential methods of mitigating the effects of incoming radioactively contaminated waters. With a member of the Orange County Water District, we obtained their opinion of what options might be available in their systems. These are presented below.

\section{Metropolitan Water Reclamation District of Greater Chicago}

The MWRD runs typical unit operations to improve wastewater for discharge to the local waterways (Figure 6-1). Discussions included ways to mitigate incoming radioactively contaminated waters. It is possible to divert water from the intake at the reclamation plant and store it in a suitable container for on-site treatment. Also, the MWRD staff explained that they can move incoming water into the Tunnel and Reservoir Plan System (Chicago Deep Tunnel) (https://www.mwrd.org/irj/portal/anonymous/tarp) by causing an overflow of their intake. This can be done by dumping material into the two interceptors (the upstream side of the overflow weirs) leading from the city of Chicago. Then, the water would be diverted into the tunnel system (1.6 billion-gallon capacity in tunnels) and reservoirs (current capacity of 11.4 billion gallons).

4 City of Chicago, "Water Management,"

https://www.cityofchicago.org/city/en/depts/water/supp_info/education/water_treatment.html, accessed June 2018. 


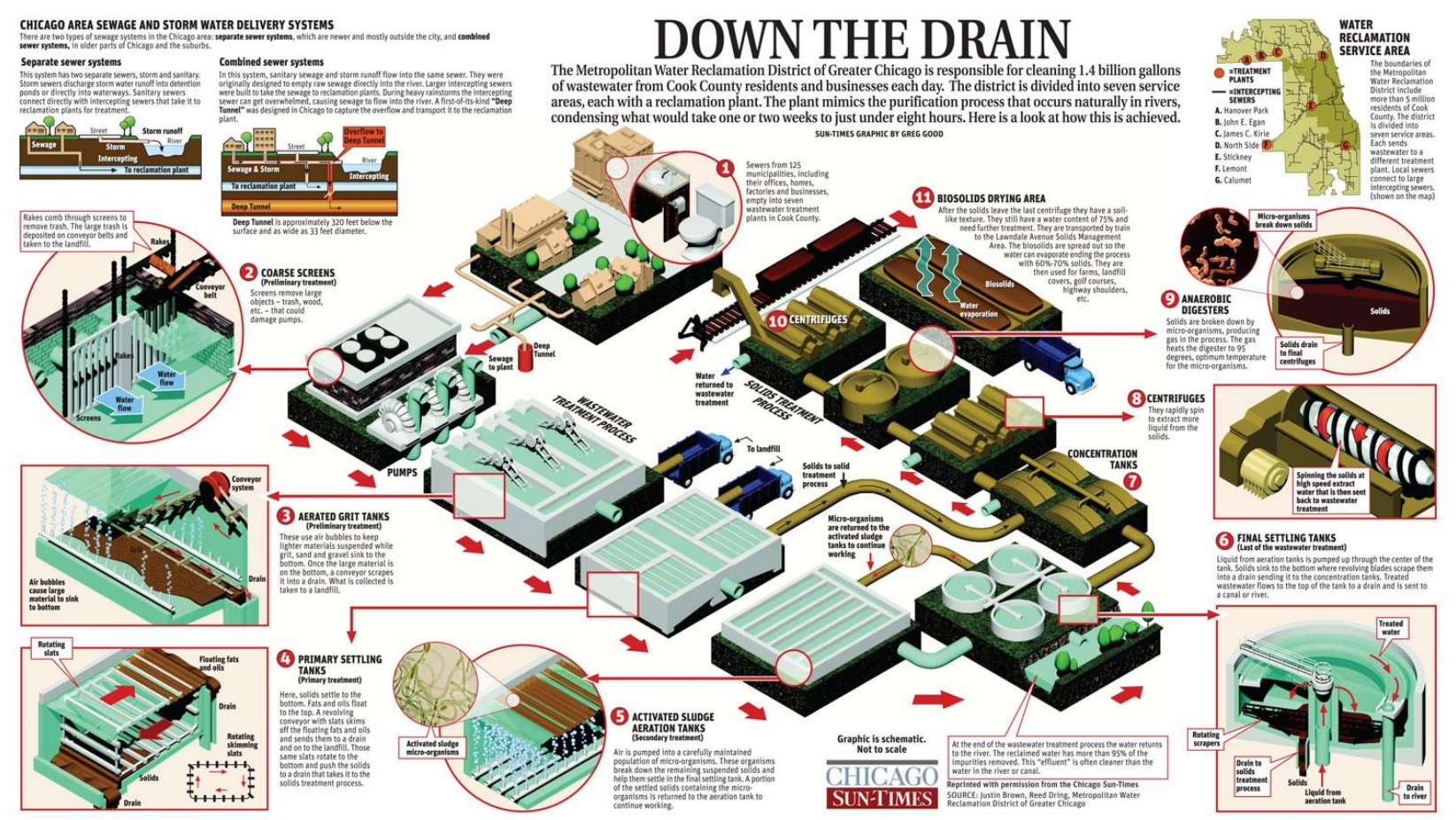

FIGURE 6-1 MWRD Stickney Plant flow diagram. The Stickney Plant is one of seven reclamation plants operated by the MWRD (image courtesy of Metropolitan Water Reclamation District of Greater Chicago).

Once the water is diverted into the tunnels, there may be options to pump and treat the water at the reservoirs or within the tunnel system itself and discharge the treated water back to the reclamation plant. However, the MWRD staff did not recommend dropping solids into the reservoirs to settle the radionuclides and then pump the treated water to the reclamation plant. Instead, to facilitate removal of the radioactive particles and solution, several possibilities were discussed. A sequestration agent (e.g., clay) could be added into the sewer system to react with the contaminated water and sorb the radioactivity onto the clay surface. Then, once the water reaches the reclamation district, the clay can be settled out in the initial unit operations at the plant.

One method might be to add the sequestration agent into sewer inlets on the streets within the contaminated zone and let the slurry mix and react as the flow travels to the reclamation plant. Then, at the plant, the solids are separated. The clean water would eventually discharge to the canal, and the solids would be stored in existing tanks to be treated and/or removed (there is a separate pump station and pipes for removal of these solids).

Another option is to add the sequestration agent directly into the preliminary tanks (settling) and use the primary settlers to be the reactor vessels. The MWRD staff noted that at maximum flow the residence time is 30 minutes, and at low flow periods the rate is 2 hours. Before the primary settler, there is a grit removal tank where some mixing action occurs that can be used to mix the sequestration agents. Adequate space for access by trucks to dump the sequestration agent would need to be established, or the MWRD may employ a conveyer system to meter in the 
sequestration agents. Figures 6-2 to 6-4 show the unit operations mentioned above for one of the treatment trains at the Stickney facility.

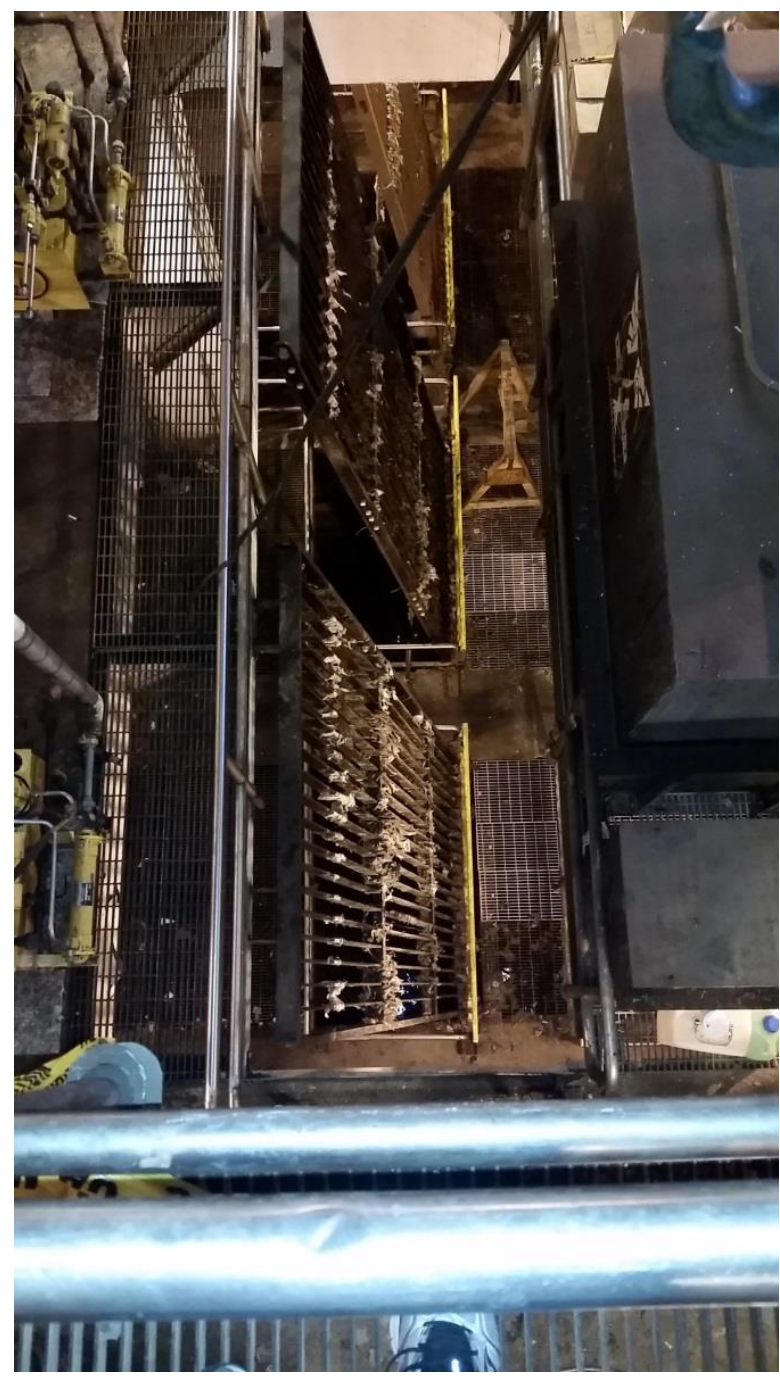

FIGURE 6-2 A look from above the coarse screening operations. Here, the water is agitated via aeration to promote the capture of large debris onto the coarse screens.

Sequestering agents can be introduced here via truck or conveyor system and mixed via aeration pumps to promote sorption of radionuclides on the sequestering agents. These finer sequestration agent solids would pass onto the aerated grit tanks.

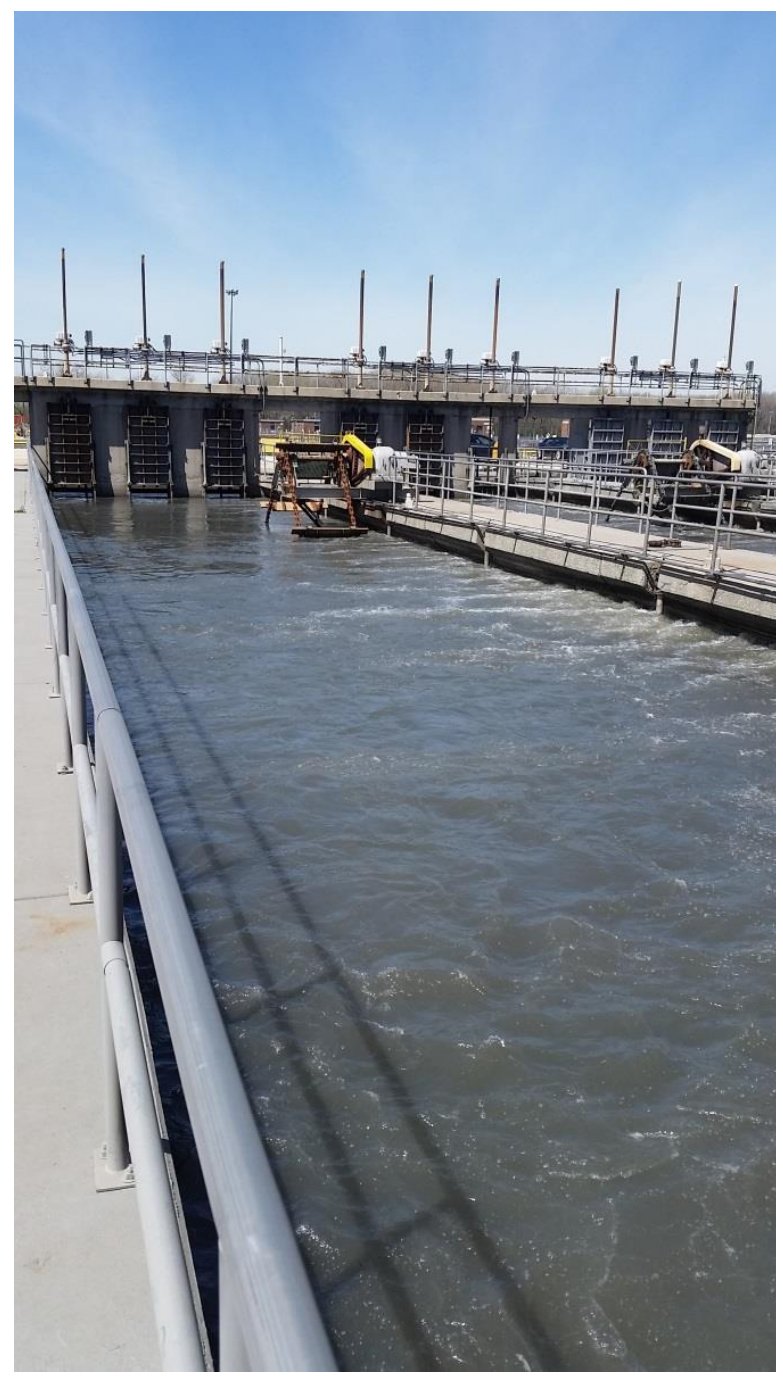

FIGURE 6-3 Water level view of the aerated grit tanks that can be used to remove the bulk of sequestering agents introduced in the sewer system or in the coarse screen houses.

Aeration pumps drive the fluid upward at the right hand side of the photo and cause the fine solids to descend at the left-hand side of the photo to the sloped bottom. The fines collect at the bottom right of the photo, below the aeration pumps. The initially clarified liquid passes to the primary settling tanks. 


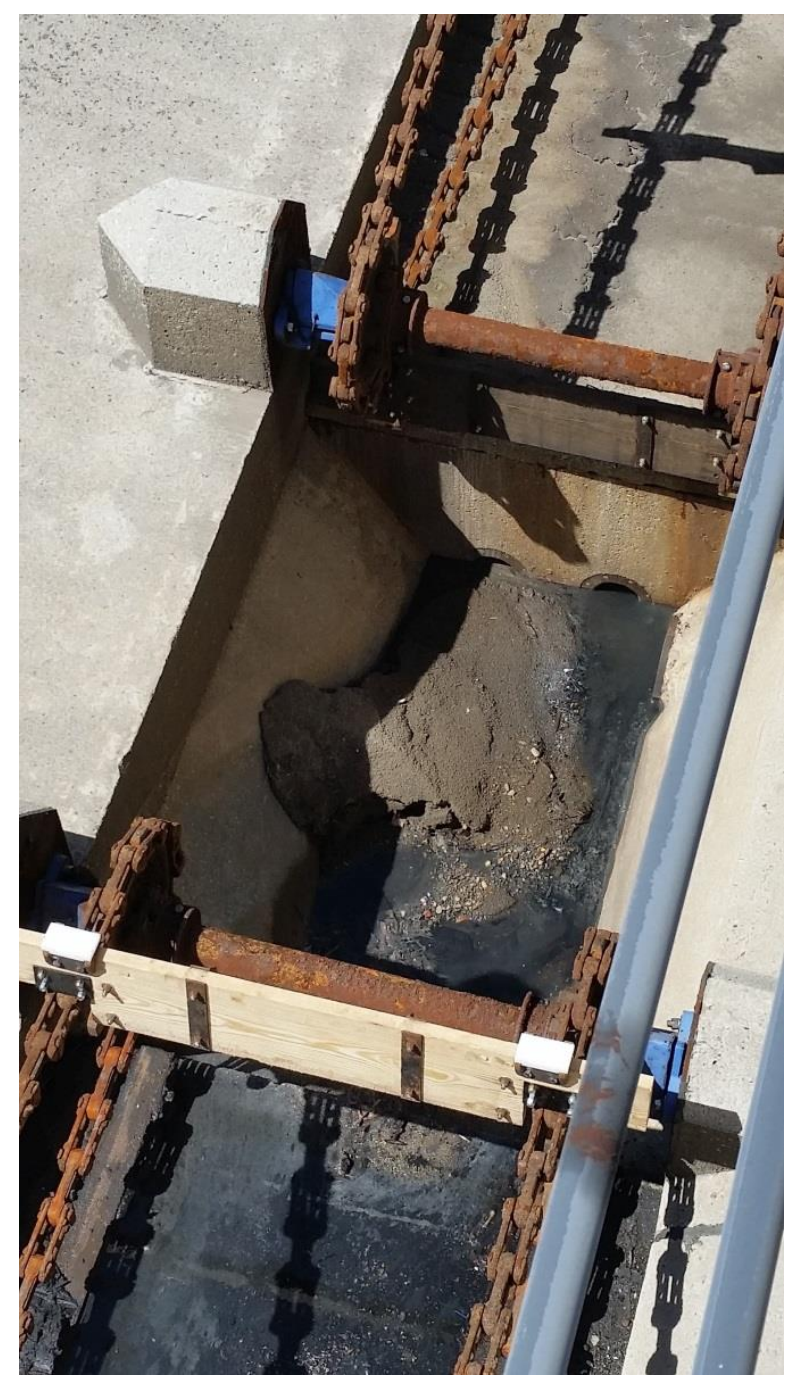

FIGURE 6-4 Close-up view of the collection trench in a drained aeration grit tank. Note the wood scrapers that drag the fines collected at the bottom of the sloped trench and force them into the collection trench where they are pumped for disposal.
When asked if the District has the ability to divert water directly into the discharge canal before it enters the plant, with the goal being to collect the water in barges located on the canal, the staff explained that they would have to close all gates that lead to the Chicago Deep Tunnel, and then it would overflow as combined sewer overflow. At the plant, they could close the gates inside the plant, then the incoming water would overflow into the canal. However, the outflow pipe into the canal is below the river level, so there is no direct way to isolate and collect that outflow.

\section{Orange County Water District}

The representative of the Orange County Water District (OCWD) suggested directing or diverting incoming water using diversion structures currently used to move noncompatible drinking water. One can use a large portable pump that would need to be leased from well flushing or other industry. OCWD was unsure how leased equipment could be decontaminated, or if it were possible. Such water could be sent to the collection system discharge. The OCWD staff raised the concern that there are no current regulations on the biosolids generated from radioactive waters. Therefore, if a radiological release event were to occur, they would rapidly accumulate the biosolids and quickly run out of space.

According to the State of California Proposition 45, the public needs to be notified regarding contents that are being discharged and transported, so developing the appropriate signage might be a potential concern. In addition, if the initial solids are radioactive, then the OCWD might need to re-designate the waste and make appropriate changes to its transport.

When asked if their plant can substitute out their resins systems with selective resins for radionuclides, the OCWD staff explained that their advanced treatment system at the plant has reverse osmosis (RO) membrane units. Perhaps, the radionuclides would be rejected at the RO unit. Beyond the RO, they have an oxidation process. In the large municipal systems, there might be a chance to employ a pillow plug and Baker tanks, trailer or railcar mounted tanks can be used to temporarily hold flow (as a pump-around) and return the water into the system after initial diversion and cleaning. 
The OCWD has inflatable tanks/dams that are permanent to the storm water pipe systems (Figure 6-5). It is possible to hold tremendous amounts of water in the concrete lined riverbeds (like Santa Ana riverbed) to treat and then discharge the water. The structures may require decontamination. Some of these lined riverbeds are used for recreation activities, so some provisions would need to be made.

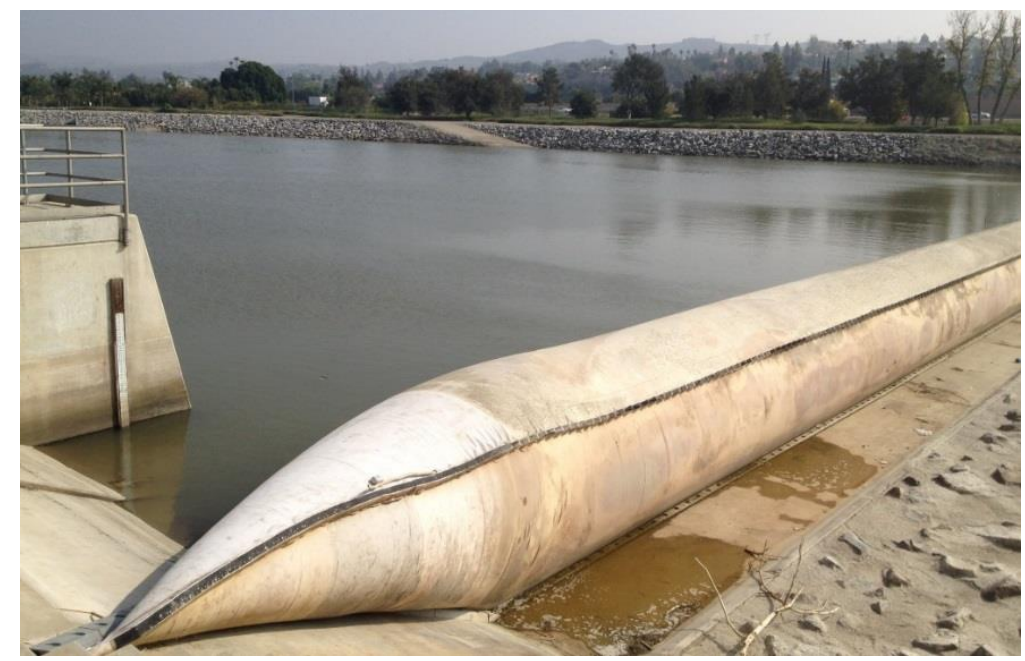

FIGURE 6-5 OCWD inflatable rubber dam spanning the Santa Ana River in Anaheim to divert river water that would otherwise flow to the ocean. The water flows into one of the district's recharge basins, where it eventually percolates into an underground aquifer that supplies water to 2.4 million Orange County residents.

\subsection{HOSPITALS}

Although we did not have the opportunity to meet with representatives of hospitals, we consulted the Guidelines for Design and Construction of Hospital and Health Care Facilities (Facility Guidelines Institute, 2006). Importantly, a common theme in the design of hospitals is the use of non-absorptive surfaces (Figure 6-6) that are durable to physical wear and chemical treatment using cleaning solutions and that minimize the number of joints and joint gaps size. These features should facilitate decontamination of radiological agents.

Ventilation rates in hospitals vary from 2 to 15 air exchanges per hour and 2-3 minimum air changes of outdoor air per hour ("To satisfy exhaust needs, replacement air from the outside is necessary") with directed inflow from the cleanest patient care areas to the less clean areas. Protective environment rooms are protected by HEPA filters at $99.97 \%$ efficiency for a $0.3 \mu \mathrm{m}$-sized particle. Therefore, airborne contamination entering the ventilation system may or may not pose a hazard depending on the specific air handling systems employed. 

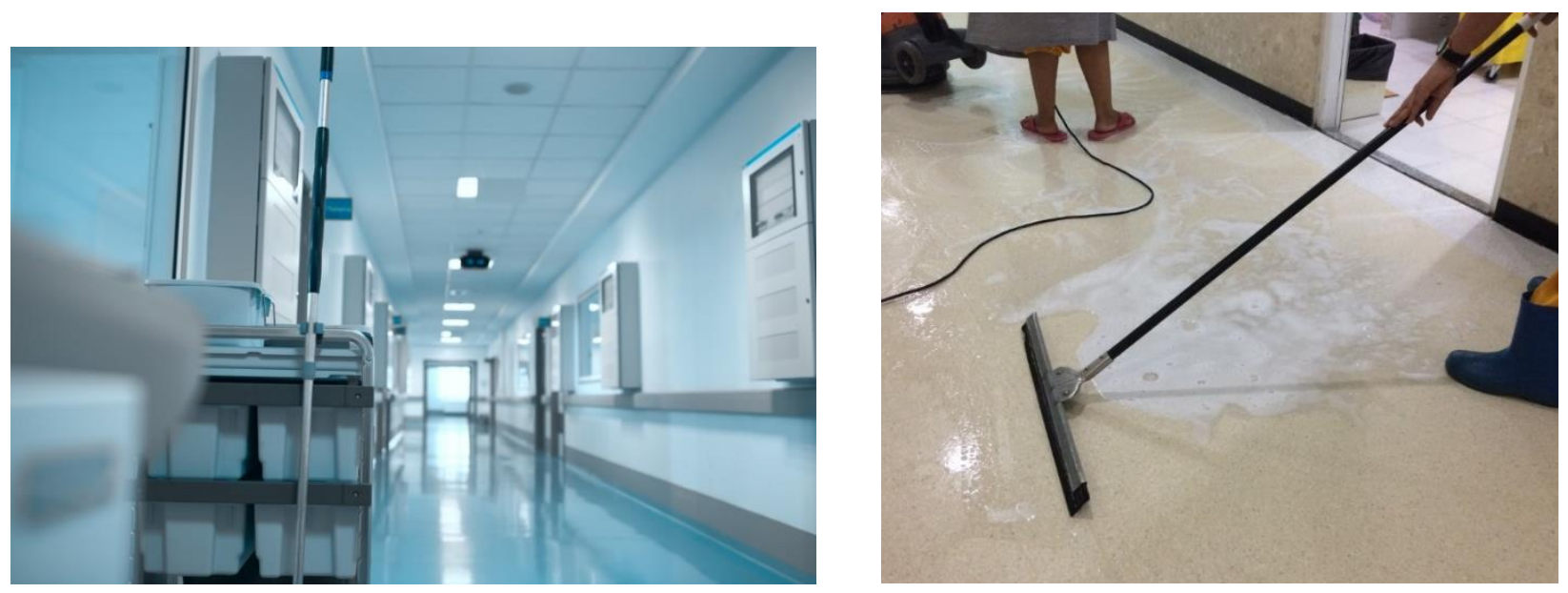

FIGURE 6-6 Hospital design and cleanup. Hospitals are designed for facile clean up using common industry practices to minimize transfer of disease. Such practices are compatible with decontaminating radioactive contaminations on surfaces (images courtesy of Shutterstock).

\subsection{SEAPORTS}

There was no opportunity to consult with representatives at the cargo container ports (Figure 6-7) around the country. However, the surfaces encountered at the port are not unlike those in an urban area. Methods would have to be developed to decontaminate the many cargo containers and manage the radioactive liquid and solid waste. Also, the seaports provide a potential means of transporting large amounts of contaminated debris, provided there is a process to move these materials in accordance with the rules and restrictions for transport of radioactively contaminated materials.

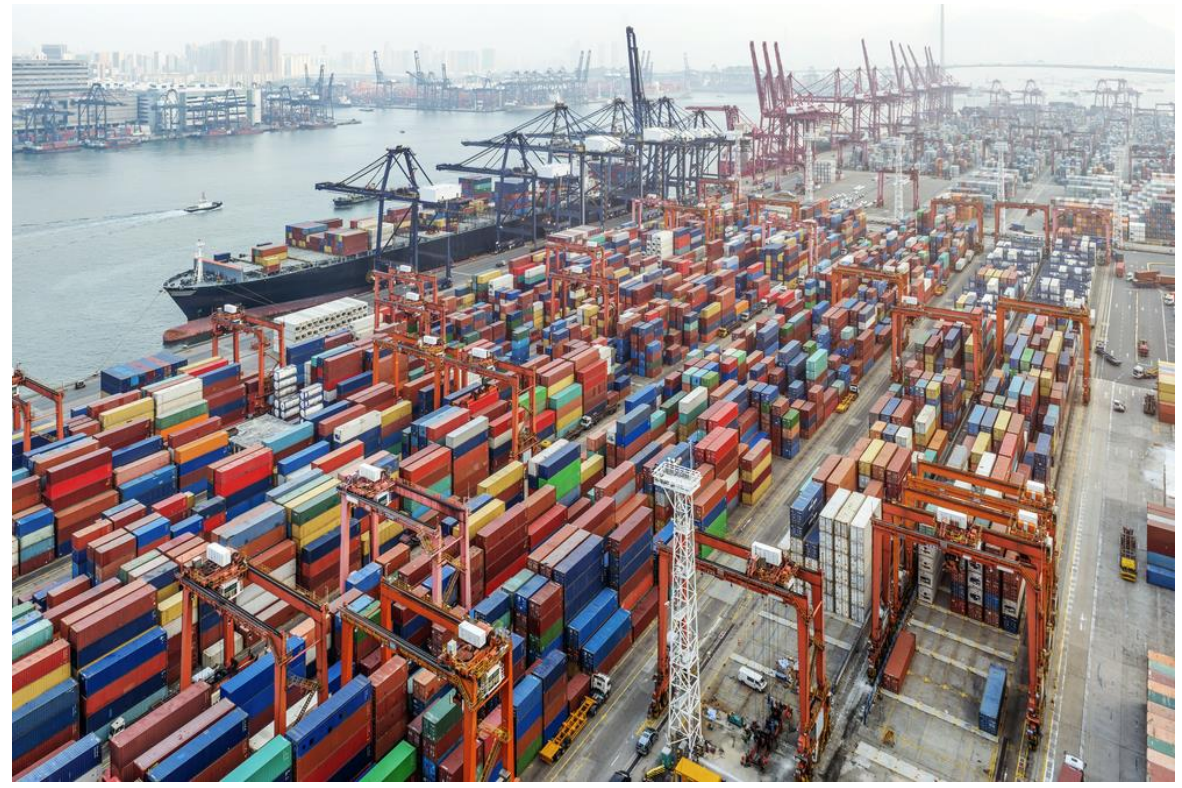

FIGURE 6-7 View of a cargo container port (image courtesy of Shutterstock). 


\subsection{AIRPORTS}

There was no opportunity to consult with representatives at the various airport authorities around the country. We do note that their building and roadway infrastructure (Figure 6-8) is not unlike those found in an urban area. In addition, they have their own emergency capabilities with commensurate equipment assets such as pumps, sprayers, snow and debris removal equipment, and asphalt repair and amendment systems. The large airports often also have direct access to rail and truck transport systems. Therefore, containment of radioactivity and decontamination of the airports may benefit from their own capabilities, assets, and relatively simple design features (i.e., long, flat surfaces). Moreover, the airports provide a means of sending and receiving equipment assets to support a response and recovery effort.

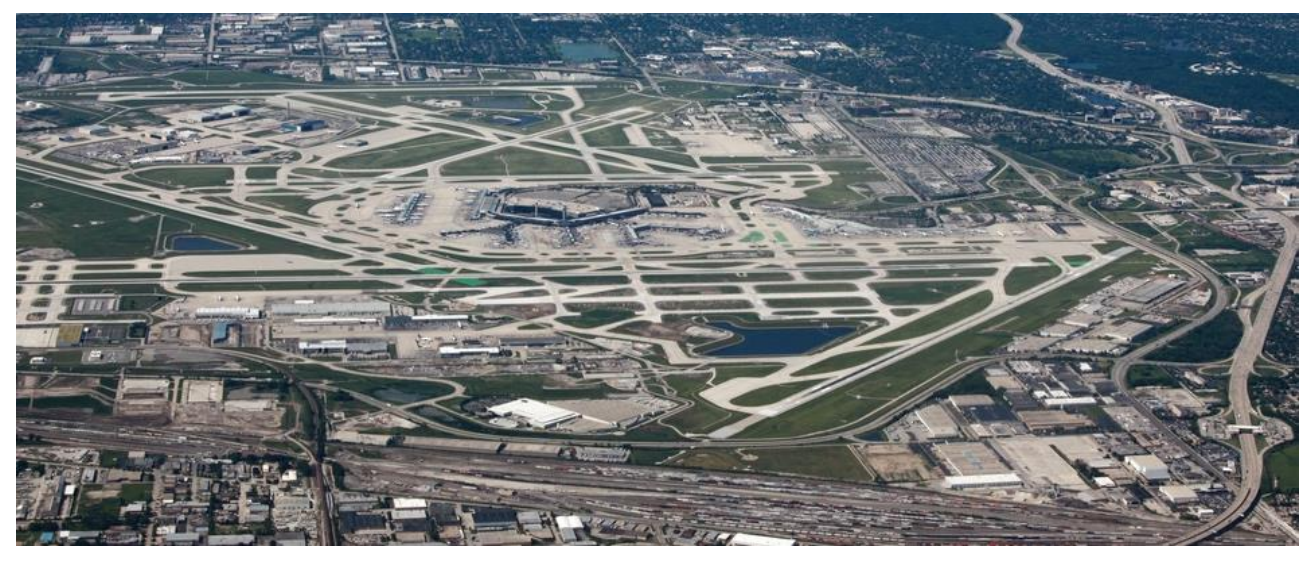

FIGURE 6-8 View of a large international airport located in the United States (image courtesy of Shutterstock).

\subsection{ELECTRICAL DISTRIBUTION}

No specific subject matter experts were consulted or review of documents related to the infrastructure of electrical distribution (Figure 6-9) was conducted at this time. 


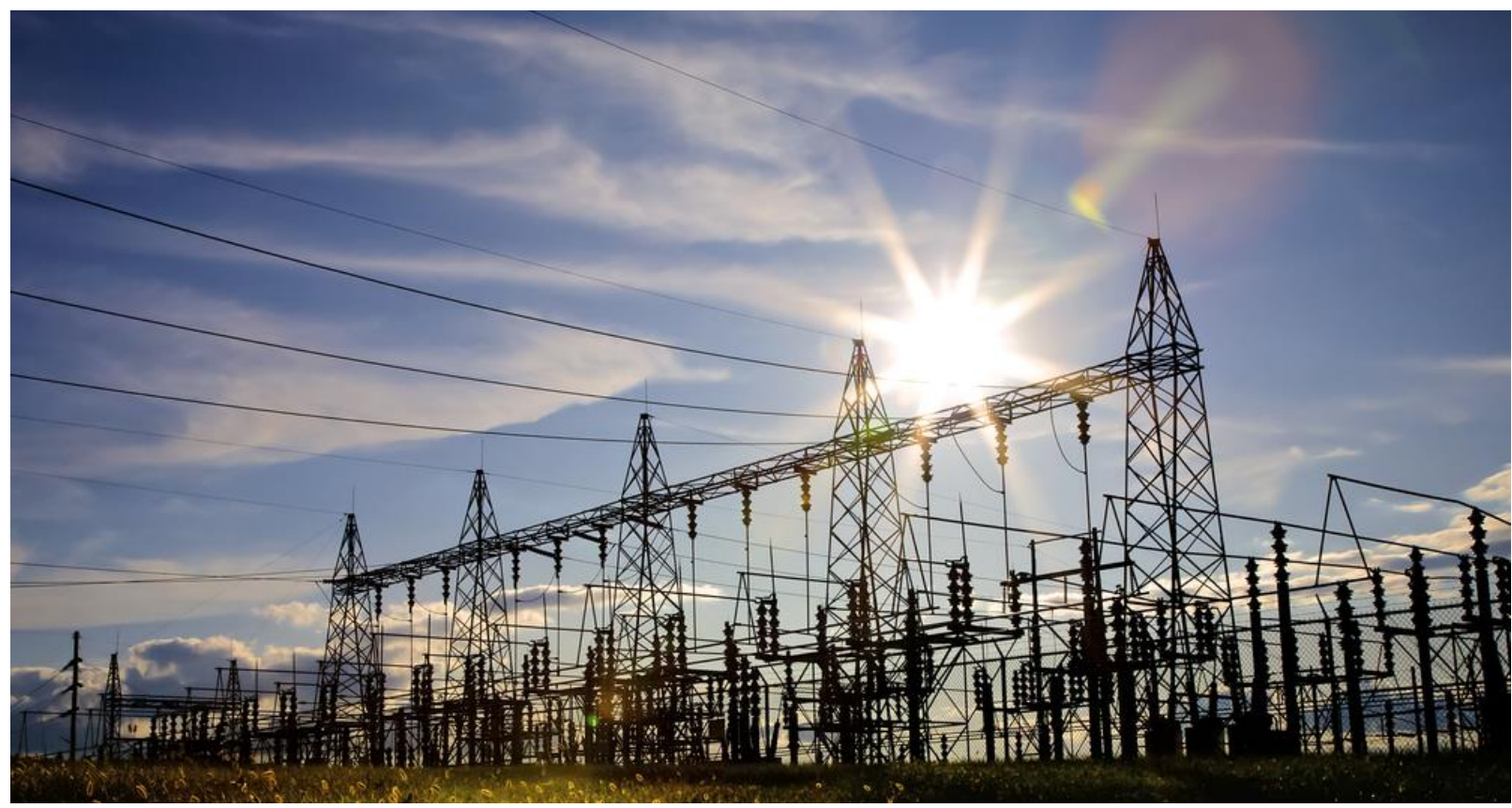

FIGURE 6-9 View of electrical distribution substation (image courtesy of Shutterstock).

\subsection{NATURAL GAS SUPPLY}

No specific subject matter experts were consulted or review of documents related to the infrastructure of natural gas supply was conducted at this time.

\subsection{PETROLEUM REFINERY}

No specific subject matter experts were consulted or review of documents related to the infrastructure of petroleum refining (Figure 6-10) was conducted at this time. 


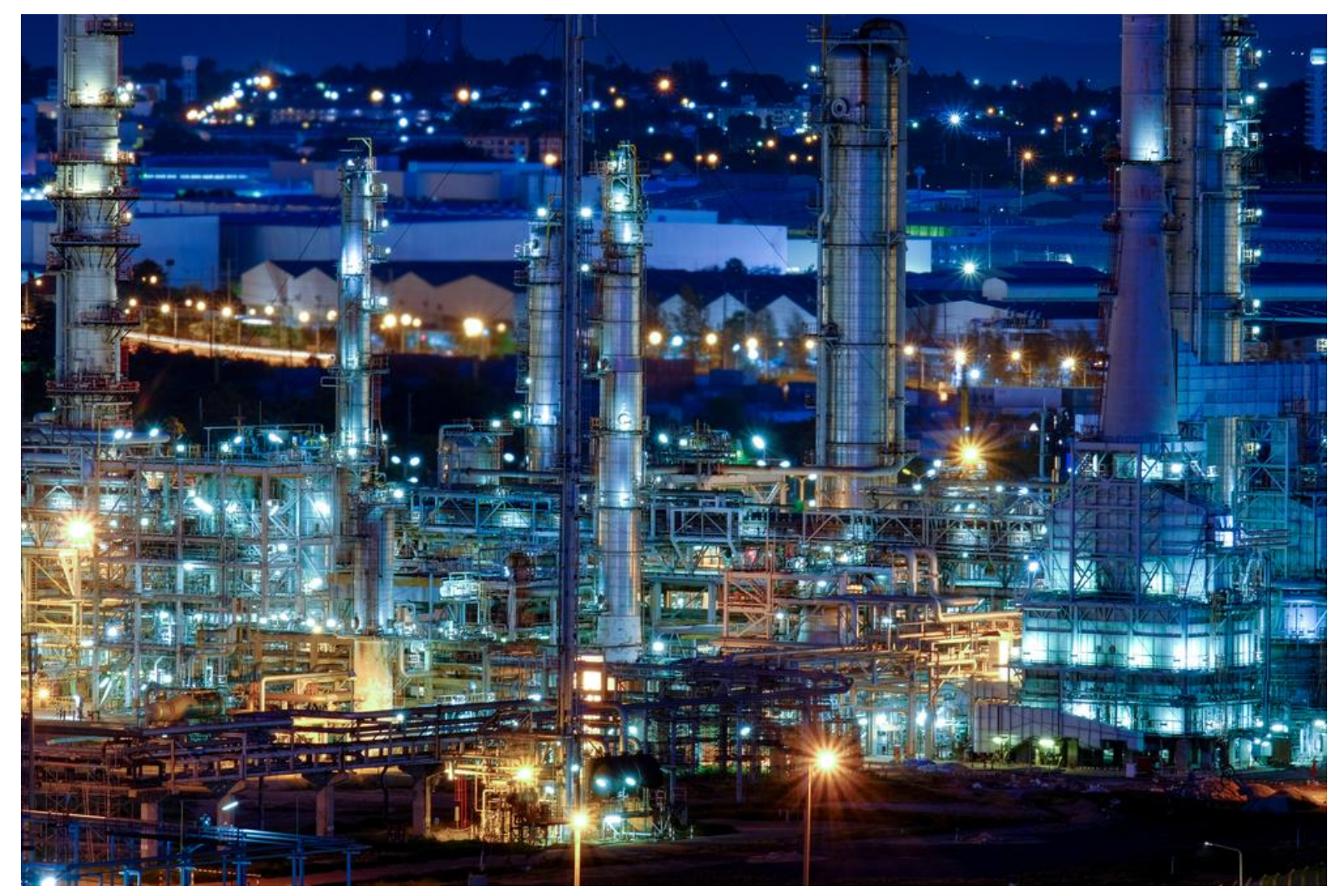

FIGURE 6-10 View of a petroleum refinery (image courtesy of Shutterstock).

No specific subject matter experts were consulted or review of documents related to the infrastructure of petroleum ocean or river terminals (Figure 6-11) was conducted, although surfaces encountered at the terminals are not unlike those in an urban area. 


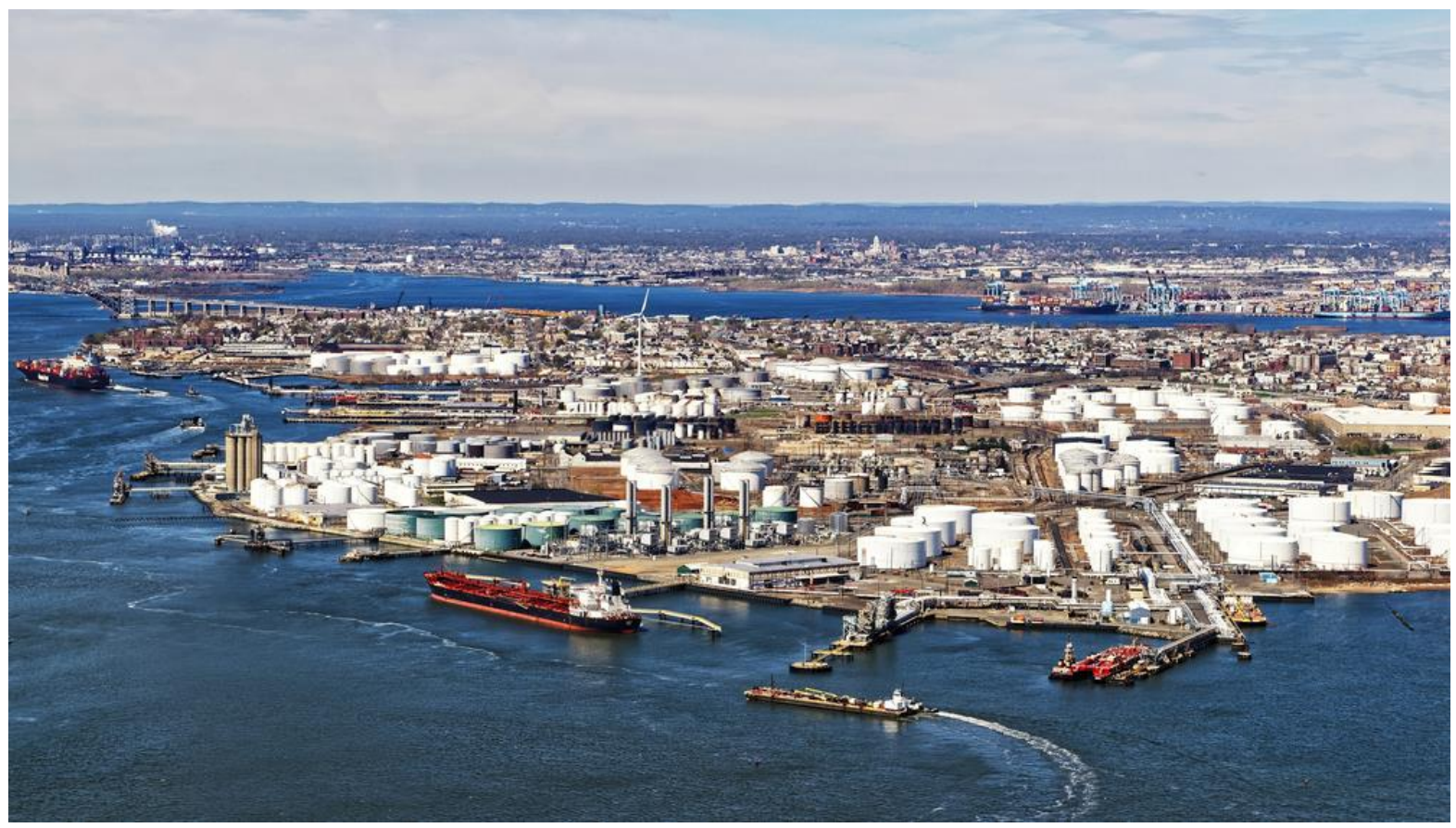

FIGURE 6-11 View of a large petroleum terminal (image courtesy of Shutterstock).

\subsection{INTRA-CONTINENTAL SHIPPING}

No specific subject matter experts were consulted or review of documents related to the infrastructure of intra-continental shipping (Figure 6-12) was conducted at this time, although surfaces encountered at the terminals are not unlike those in an urban area. These terminals also provide an integrated network from which to send and receive assets to support a response and recovery effort. 


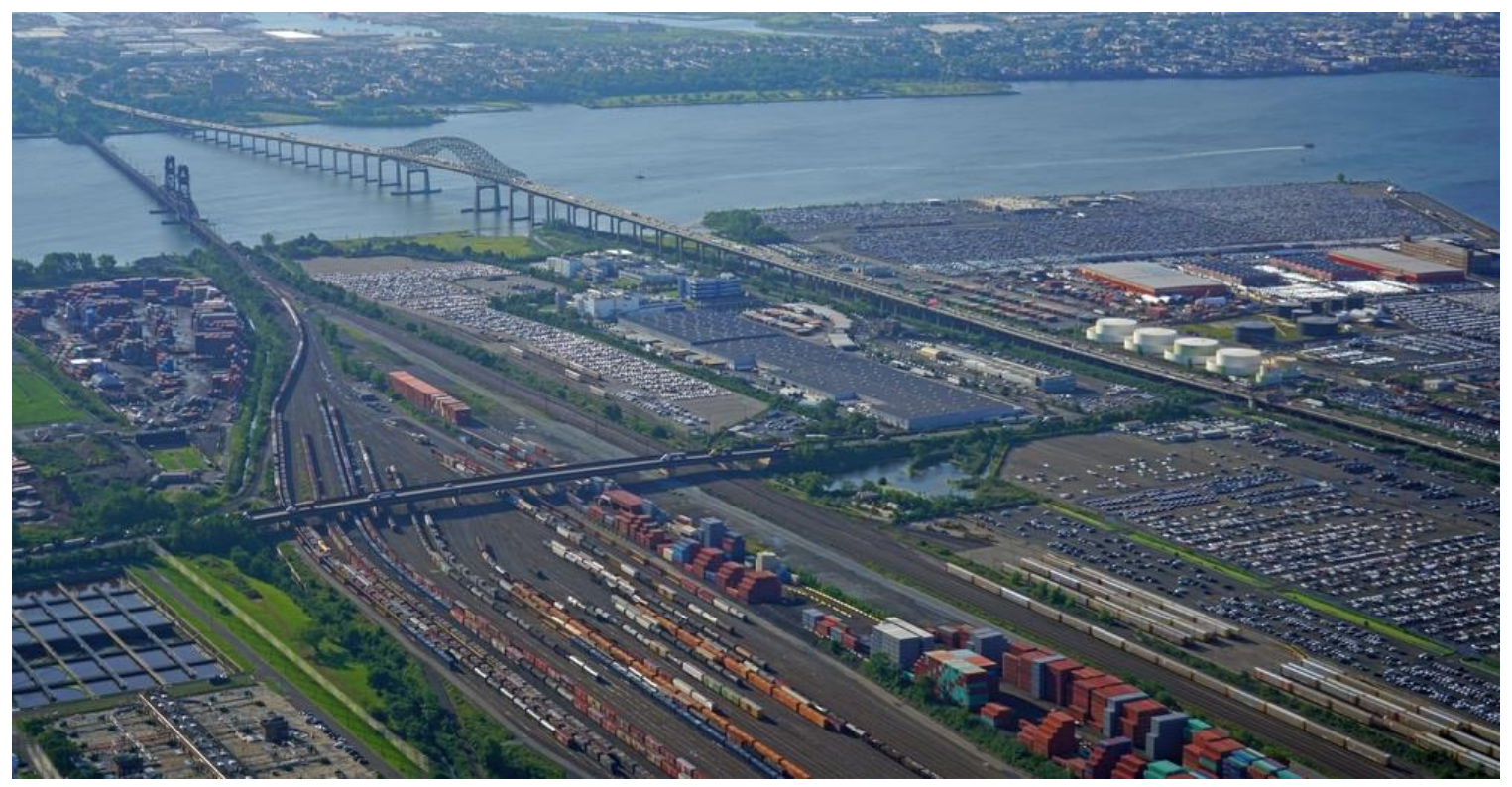

FIGURE 6-12 Views of inland waterway port and rail yard for the movement of intracontinental goods (image courtesy of Shutterstock).

\subsection{CRITICAL INFRASTRUCTURE EVALUATION CONCLUSIONS}

This exercise of providing a brief evaluation of critical infrastructure shows that many technologies and approaches to mitigating the effects of radioactive contamination developed in Sections 3-4 of this report apply to the structures (e.g., building materials, transport vehicles, water) found in critical infrastructure. Moreover, there is potential to utilize capabilities of critical infrastructure in the radioactive response (e.g., contain water, transport supplies, remove debris) and to design the radioactive response to be most effective given the unique features of the infrastructure itself (e.g., facile decontamination of hospital indoor surfaces, front-end water treatment systems at waste water treatment facilities). 


\section{CONCLUSIONS}

This report provides a thorough evaluation of technologies and equipment assets that may be effective at mitigating the effects of a wide-area radioactive contamination event and to restore critical functions to the urban environment. Moreover, equipment that could facilitate decontamination efforts during a recovery phase are evaluated. The summary of methods has been critically evaluated in terms of equipment availability, potential advantages and limitations, and research needs in order to justify and predict its efficacy. By ranking the host of techniques, a concise list of those technologies is provided that might be most impactful to a response and recovery effort and how to direct future effort. The totality of this effort and future work anticipates providing early responders and stakeholders with practical guidance information to expedite an effective response and recovery effort and mitigate the potentially devastating effects of a wide-area radioactive contamination event. 


\section{Argonne}

Nuclear Engineering Division

Argonne National Laboratory

9700 South Cass Avenue, Bldg. 208

Argonne, IL 60439-4854

www.anl.gov

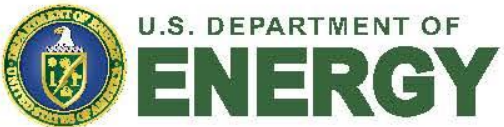

Argonne National Laboratory is a U.S. Department of Energy

laboratory managed by UChicago Argonne, LLC 